\title{
A robust and computationally efficient finite element framework for coupled electromechanics
}

\author{
Chennakesava Kadapa ${ }^{\mathrm{a}, *}$, Mokarram Hossain ${ }^{\mathrm{b}, *}$ \\ ${ }^{a}$ Swansea Academy of Advanced Computing, Swansea University, SA1 8EN, United Kingdom \\ ${ }^{b}$ Zienkiewicz Centre for Computational Engineering (ZCCE), Swansea University, SA1 8EN, United Kingdom
}

\begin{abstract}
Electro-active polymers (EAPs) are increasingly becoming popular materials for actuators, sensors, and energy harvesters. To simulate the complex behaviour of actuators under coupled loads, particularly in the realm of soft robotics, biomedical engineering and energy harvesting, finite element simulations are proving to be an indispensable tool. In this work, we present a novel finite element framework for the simulation of coupled static and dynamic electromechanical interactions in electro-active polymeric materials. To model the incompressible nature of EAPs, a two-field mixed displacement-pressure formulation which, unlike the commonly-used mixed three-field and $\boldsymbol{F}$-bar formulations, is applicable for both nearly and fully incompressible materials, is employed. For the spatial discretisation, innovative quadratic Bézier triangular and tetrahedral elements are used. The governing equations for the coupled electromechanical problem are solved using a monolithic scheme; for elastodynamics simulations, a state-of-the-art implicit time integration is adapted. The accuracy and the computational efficiency of the proposed framework are demonstrated by studying several benchmark examples in computational electromechanics which include simulations of a spherical gripper in elastostatics and a dielectric pump in elastodynamics. Such complex simulations clearly depict the advantages of the proposed finite element framework over the Q1/P0 and Q1-F-bar elements. Furthermore, the superiority of the proposed framework in accurately capturing complex coupled electromechanical interactions in thin electro-active polymeric shells is demonstrated by studying a thin helical actuator under different excitation frequencies and by reproducing buckling instabilities in thin semi-cylindrical and semi-spherical shells. With the ability to simulate various elastostatics and elastodynamics phenomena using a single finite element framework for bulk as well as thin dielectric elastomers while using coarse structured or unstructured meshes that can be readily generated using existing mesh generators, this novel framework offers a robust, accurate, and computationally efficient numerical framework for computational electromechanics.
\end{abstract}

Keywords: Electro-active polymers; Electromechanics; Smart materials; Incompressibility; Finite element analysis; Bézier elements

\section{Introduction}

Electro-active polymers (EAPs) are relatively a new class of functional or active materials that can be activated by electric fields or potential differences. A dielectric elastomer (DE), a subset of EAPs, is produced in the form of a thin polymeric film that is sandwiched between two compliant electrodes. When a voltage difference is applied across the film thickness, the material is contracted in the direction of the applied voltage and expands in the transverse planar direction; this behaviour can be termed as the mechanical output as a result of an electric input. Such activation mechanisms result in very large and complex deformations which make EAPs one of the most promising active materials for a large variety of engineering applications, e.g., artificial muscles in soft robotic mechanisms, optical membranes for shape correction in lenses, energy harvesting from ambient motions such as human walking and ocean waves [5, 17, 48, 42, 92, 93]. Due to the interplay of the mechanical and the electric field, the system of governing equations needs to be solved in coupled forms, cf. $[19,20,21,30,95,90,91]$. For a range of comprehensive reviews on the potential applications for EAPs,

\footnotetext{
${ }^{*}$ Corresponding authors

Email addresses: c.kadapa@swansea.ac.uk (Chennakesava Kadapa), mokarram.hossain@swansea.ac.uk (Mokarram Hossain)
} 
the reader is referred to recent papers, e.g., $[6,26,51,18]$. The mathematical foundations of the coupling of electromagnetic fields in finite strains are well documented in some earlier publications, see for example the works of Pao and Nemat-Nasser [66], Eringen and Maugin [28, 59], Kovetz [41] and Maugin [57]. More concise mathematical frameworks on electromechanical problems can be found in the recent monographs of Dorfmann and Ogden [22], Santapuri and co-workers [78, 79, 80].

One of the earliest experimental works that illustrates electric-field actuated large deformations is due to Pelrine et al. [68]. Commercially available acrylic type VHB, polyurethanes and silicone polymers are most commonly used as EAPs. However, due to limited actuation of unfilled polymers, high permittivity particlefilled polymeric composites are increasingly being explored for EAPs, see Madsen et al. [51], Ellingford et al. [26], Brochu and Pei [6], and Dang et al. [18], for exhaustive reviews on the state-of-the-art of EAP materials. Experimental works related to either pure or composite EAPs are very limited in the literature. Wissler et al. [100] conducted experiments on an acrylic type polymer under uncoupled electromechanical loading while Diaconu et al. [23, 24] studied the electromechanical properties of a synthesised polyurethane elastomer filmbased polyester. To illustrate the time-dependent viscoelastic behaviour of VHB 4910 polymer under pure mechanical loading, comprehensive experimental studies were presented by Hossain et al. [35], and Ahmad and Patra [3]. Furthermore, a comprehensive characterisation of the electromechanically coupled properties of VHB 4910 polymer was recently conducted by Hossain et al. [36] and Mehnert et al. [56]. Qiang et al. [69] presented an experimental study on the dielectric properties of a polyacrylate dielectric elastomer while Michel et al. [58] performed a comparison between silicone and acrylic elastomers that can potentially be used as dielectric materials in electro-active polymer actuators. Note that frequently used commercially available acrylic-based VHB polymer has a limited actuation that motivates Zhao et al. [101, 102] to formulate new acrylic type polymers which can be actuated at a very low electric voltage.

Thanks to a wide range of applications of EAPs, the constitutive modelling of electro-elasticity at finite strains has been an active field of research for more than two decades. To capture electromechanical coupled behaviour at large strains, in a series of papers, Dorfmann and Ogden $[19,20,21]$ and Bustamante $[8,9,10$, 82] proposed constitutive models of isotropic electro-elasticity which are mainly based on several coupled invariants. To enhance actuations, EAPs are frequently filled with high electric permittivity fillers which are further cured under an electric field during the curing process that results in anisotropic EAP composites, see Kashani et al. [43], Carpi et al. [14] and Kumar et al. [44] for experimental evidences, and refer to Keip and coworkers $[45,70]$ and Ullah et al. [71, 72] for homogenization-based simulation frameworks. In order to capture the underlying inhomogeneous behaviour of particle-filled EAPs, Bustamante [8], Hossain and Steinmann [33], Hossain [34], Sharma and Joglekar [73, 74], Bustamante and Shariff [11] and Shariff et al. [81] proposed mathematical formulations of electro-elasticity that incorporated transverse and dispersion-type anisotropy. All the constitutive modelling frameworks mentioned above are based on the so-called phenomenological approach. In contrary, exploiting the micro-mechanics of polymeric chain orientations and deformations under mechanical and electromechanical coupled loads, Itskov et al. [39], Cohen et al. [15, 16], Brighenti et al. [4], and Thylander et al. [87, 88] proposed micro-mechanically-motivated electro-elastic models at finite strains with physically meaningful material parameters. Other efforts in formulating constitutive modelling of electro-elasticity are due to Gao et al. [30], Zhao and Suo [98], Henann et al. [32], Ask et al. [1], Büschel et al. [12], Nedjar [60], Wang et al. [99], Sharma and Joglekar [74], Wang et al. [99], Thylander [87], Vogel et al. [90, 91], Saxena et al. [76, 77], Skatulla et al. [83], and Zäh and Miehe [97].

Similar to the constitutive modelling of EAPs, the finite element (FE) implementation of the electromechanically coupled problem is an active area of research in recent years. One of the earliest seminal works in the area of FE simulations of electro-elastic problems is due to $\mathrm{Vu}$ et al. [94]. Variational formulations for the governing equations of the coupled electromechanical problems are extensively considered in the works of Bustamante et al. [10] and Vogel [90] using a three-field formulation. Note that most of the earlier numerical formulations of electromechanics neglected the free space contribution which plays a significant role in deforming EAP structures. Hence, very recently, Vu and Steinmann [95, 96], Pelteret et al. [67] and Nedjar [61] provided computational frameworks for nearly incompressible electro- and magneto-elastic solids immersed in a free space. Since dielectric elastomers are mainly made of thin structures, a solid shell finite element formulation using a mixed variational principle of Hu-Washizu type is proposed by Klinkel et al. [46]. In order to ameliorate volumetric-locking, a common phenomenon in nearly incompressible rubber-like materials, Hennan et al. [32] extended the classical $\boldsymbol{F}$-bar element method proposed by de Souza Neto et al. [25] 
to the case of electro-elastic coupled problem while Sharma and Joglekar [74] extended the $J$-bar method [123] for simulating the nearly incompressible electro-viscoelasticity. Very recently, in a series of papers, Gil and co-workers [31, 62] proposed a new constitutive framework with finite element implementations for large strain electromechanics based on the so-called convex multi-variable strain energies. Furthermore, Bishara and Jabareen $[7,40]$ proposed a numerical scheme using the reduced mixed finite element formulation, which eliminates the possible volumetric locking in electro-active polymers and enhances the computational efficiency as the static condensation is circumvented.

Note that all of the above FE frameworks for electromechanically coupled simulations discard inertial effects in the governing equations. Dynamical simulations of EAPs are largely ignored in the literature. However, inertia effects are essential to capture physical details that may arise during the electromechanical softening and instability of the dielectric elastomers. In a series of papers, Park and co-workers $[63,64,65,85]$ simulate various dynamic instabilities such as wrinkling, creasing in the EAPs using fully-coupled three-dimensional formulations either by a monolithic or a staggered solution scheme. In a similar effort to obtain the timedependent behaviour of EAPs, Schlögl and Leyendecker [89] developed a finite element framework where an energy-conserving integrator is used to discretise dynamical equations. Experimental evidence suggests that polymeric materials are inherently prone to temperature fluctuations which are almost impossible to prevent during their application due to the high electric voltages and various dissipation mechanisms responsible for temperature variations [49, 50, 86]. In the works of Vertechy et al. [92, 93], Chen [13], Liu [47] and Erbts et al. [27], theoretical frameworks to the thermo-electromechanical problem can be found. Recently, Mehnert et al. $[52,53,54]$ proposed a framework for thermo-electro-elasticity which was later extended to the case of finite strain thermo-magneto-mechanical problems in Mehnert et al. [55]. However, these numerical treatments of thermo-electro-elasticity are based on traditional displacement-based formulations, hardly suitable for purely incompressible materials like EAPs.

In spite of the tremendous success by various researchers in extending the selective reduced integration method, three-field, $\boldsymbol{F}$-bar and $J$-bar formulations with the Q1 element for computational electromechanics, a numerical framework that is accurate, robust and also computationally efficient is still lacking. This is primarily due to the limitations of these formulations that become significant bottlenecks when applied to incompressible materials, especially when the Poisson's ratio approaches 0.5 . The issues and limitations of widely-used finite element schemes in the computational electromechanics community are:

- The $\boldsymbol{F}$-bar formulation, despite its effectiveness for modelling nearly incompressible hyperelastic and elastoplastic materials, still requires as many load steps as necessary for the pure displacement formulation, and this number increases as the Poisson's ratio approaches 0.5. Such an increased computational cost of the $\boldsymbol{F}$-bar method limits its accuracy in imposing the incompressibility constraint and its applicability to large-scale real-world problems. The same arguments are also applicable to the $J$-bar formulation because of the fundamental similarity of the $\boldsymbol{F}$-bar and $J$-bar formulations in retaining the volumetric part of the energy function in the elastic tangent tensor.

- The three-field (displacement-pressure-Jacobian) formulation with the Q1/P0 element for purely mechanical cases [115], while is effective in dealing with the volumetric locking and also requires significantly fewer load steps when compared with the pure displacement, $\boldsymbol{F}$-bar and $J$-bar formulations, suffers from spurious pressure modes and serious convergence issues when $\nu \rightarrow 0.5$, as demonstrated with the example of a spherical gripper in Section 5.4. The spurious pressure modes are a direct consequence of the fact that the Q1/P0 element is not inf-sup or Ladyzhenskaya-Babuska-Brezzi (LBB) stable, see [110, 116].

- All of these three formulations are not applicable for truly incompressible materials, i.e., for $\nu=0.5$.

- The use of quadrilateral and hexahedral elements in these schemes make the task of mesh generation quite cumbersome, especially for complex geometries.

Therefore, in this contribution, a new finite element framework that overcomes the above-mentioned issues and that is robust and computationally efficient for computational electromechanics, is proposed.

The proposed framework is an extension of the innovative finite element framework for computational solid mechanics based on Bèzier elements, which is very recently proposed by Kadapa [111, 112]. Considering the advantages of the two-field mixed (displacement-pressure) formulation demonstrated rigorously in Kadapa [107, 112], Kadapa et al. [108, 137] for pure mechanical cases, we propose a novel three-field formulation 
with displacement, pressure, and electric potential as the primary solution variables for the electromechanical problem. For the spatial discretisation, we use Bèzier elements because of their proven advantages over the Lagrange family of elements, see $[111,112]$. The advantages of the proposed framework are multifold, which can be summarised as:

1. The proposed work employs a two-field mixed formulation for the mechanical problem which is applicable for both nearly and truly incompressible materials unlike the three-field mixed formulation which is not applicable for the truly incompressible model.

2. This newly devised framework employs inf-sup stable elements [112] as opposed to the widely-used $\mathrm{Q} 1 / \mathrm{P} 0$ element, which is not inf-sup stable. Therefore, the pressure field computed using the proposed element is free from spurious oscillations.

3. The current work does not include any ad-hoc parameters that control accuracy and stability, making it a robust framework, unlike the mixed stabilised formulations. Moreover, unlike the Q1/P0 element, the proposed element is not sensitive to the bulk modulus.

4. This work uses triangular and tetrahedral elements which are comparatively easier to generate using automated mesh generators, even for complex geometries. It can also be extended to structured meshes using quadrilateral/hexahedral elements in a straightforward manner.

5. The proposed FE strategy uses Bézier elements which, unlike the higher-order Lagrange family of elements, are advantageous in explicit schemes for elastodynamics simulations of problems involving highstrain rates and instabilities, see [111, 112, 84].

This manuscript is the first part of a series of contributions with the ultimate goal to connect and verify a continuum modelling approach with realistic experimental data. This paper is organised as follows. The finite strain theory and the governing equations for the coupled electromechanics are presented in Section 2 , followed by a discussion on the material models used in the present work in Section 3. The finite element formulation used in the proposed work and the time integration scheme are discussed in Section 4. The accuracy and computational advantages of the proposed finite element framework are illustrated with several numerical examples for electro-elastostatic and electro-elastodynamics, respectively, in Section 5 and 6. The paper is ended with Section 7 with a summary of important contributions and an outlook to future works.

\section{Governing equations for nonlinear electromechanics}

\subsection{Deformation, strain and stress}

Let us consider an arbitrary solid body with $\mathcal{B}_{0}$ as its reference configuration. The body assumes a new configuration, say $\mathcal{B}_{t}$, under the influence of external forces. Let $\mathcal{X}: \mathcal{B}_{0} \rightarrow \mathcal{B}_{t}$ denotes the deformation mapping that takes a point $\boldsymbol{X} \in \mathcal{B}_{0}$ to a point $\boldsymbol{x} \in \mathcal{B}_{t}$. Now, the displacement field for identifying the new configuration, $\mathcal{B}_{t}$, from the initial configuration, $\mathcal{B}_{0}$, can be defined as

$$
\boldsymbol{u}(\boldsymbol{X}):=\mathcal{X}(\boldsymbol{X})-\boldsymbol{X}=\boldsymbol{x}-\boldsymbol{X}
$$

Using the definition of the displacement field in Eqn (1), several important strain measures for the finite strain continuum mechanics are defined as

$$
\begin{aligned}
\text { Deformation gradient, } & \boldsymbol{F}:=\frac{\partial \mathcal{X}}{\partial \boldsymbol{X}}=\frac{\partial \boldsymbol{x}}{\partial \boldsymbol{X}}=\boldsymbol{I}+\frac{\partial \boldsymbol{u}}{\partial \boldsymbol{X}}, \\
\text { Jacobian, } & J:=\operatorname{det}(\boldsymbol{F}), \\
\text { Right Cauchy-Green deformation tensor, } & \boldsymbol{C}:=\boldsymbol{F}^{\mathrm{T}} \boldsymbol{F}, \\
\text { Left Cauchy-Green deformation tensor, } & \boldsymbol{b}:=\boldsymbol{F} \boldsymbol{F}^{\mathrm{T}}, \\
\text { Green-Lagrange strain tensor, } & \boldsymbol{E}:=\frac{1}{2}[\boldsymbol{C}-\boldsymbol{I}],
\end{aligned}
$$

where $\boldsymbol{I}$ is the second-order identity tensor. 
Using the above strain measures, important stress measures in the finite strain theory for continuum solid mechanics that are used in the present work are defined as,

$$
\begin{aligned}
\text { First Piola-Kirchhoff stress tensor, } \quad \boldsymbol{P}: & =\frac{\partial \Psi}{\partial \boldsymbol{F}}=\boldsymbol{F} \boldsymbol{S}, \\
\text { Second Piola-Kirchhoff stress tensor, } & \boldsymbol{S}:=2 \frac{\partial \Psi}{\partial \boldsymbol{C}}, \\
\text { Cauchy stress tensor, } & \boldsymbol{\sigma}:=\frac{1}{J} \boldsymbol{F} \boldsymbol{S}^{\mathrm{T}},
\end{aligned}
$$

where $\Psi$ is the strain energy function. For the comprehensive details on the finite strain theory for continuum solid mechanics, we refer the reader to any of the standards textbooks on nonlinear finite element analysis, for example, Zienkiewicz and Taylor [103], Bathe [104], de Souza Neto et al. [105], Bonet and Wood [106], and Holzapfel [134].

\subsection{Electrostatics - Faraday's and Gauss's laws}

Two main laws are important for electro-statics which are Faraday's law and Gauss's law. To express these laws mathematically, we denote the quantities corresponding to the electrical field in the reference configuration as, electric field vector $\mathbb{E}$; electric displacement vector $\mathbb{D}$; and electric polarisation density $\mathbb{P}$. Similarly, the quantities corresponding to the electrical field in the current configuration are, electric field vector $\mathbb{E}$; electric displacement vector $\mathbb{d}$; and electric polarisation density $p$. Scalar electric potential in the current configuration and the vacuum electric permittivity are denoted, respectively, as $\phi$ and $\varepsilon_{0}$. Now, various electric quantities defined in two different configurations are related by following relations,

$$
\begin{aligned}
& \mathbb{E}=\boldsymbol{F}^{\mathrm{T}} \mathbb{e} \\
& \mathbb{D}=J \boldsymbol{F}^{-1} \mathbb{d} \\
& \mathbb{P}=J \boldsymbol{F}^{-1} \mathbb{p} \\
& \mathbb{D}=\varepsilon_{0} J \boldsymbol{C}^{-1} \mathbb{E}+\mathbb{P}=\mathbb{D}^{\epsilon}+\mathbb{P} \\
& \mathbb{d}=\varepsilon_{0} \mathbb{e}+\mathbb{p}=\mathbb{d}^{\epsilon}+\mathbb{p} .
\end{aligned}
$$

For the given energy function $\Psi$ consisting of mechanical and electro-mechanical coupled parts, the electric displacement vector in the reference configuration $(\mathbb{D})$ is defined as

$$
\mathbb{D}:=-\frac{\partial \Psi}{\partial \mathbb{E}}
$$

Now, using the above-listed quantities for the electrical field, the Faraday's and Gauss's laws in the current configuration can be expressed as

$$
\begin{aligned}
\operatorname{curl} \mathbb{e} & =\nabla_{\boldsymbol{x}} \times \mathbb{e}=\mathbf{0}, \\
\operatorname{div} \mathbb{d} & =\nabla_{\boldsymbol{x}} \cdot \mathbb{d}=0 .
\end{aligned}
$$

where $\nabla_{x}$ is the gradient operator with respect to the current configuration. Equation (16) implies the existence of a scalar electric potential such that

$$
\mathbb{E}=-\operatorname{grad} \phi=-\nabla_{\boldsymbol{x}} \phi
$$




\subsection{Equilibrium equations}

A complete set of governing equations for the coupled electro-elastodynamics problem in the current configuration can be written as

$$
\begin{aligned}
\rho \boldsymbol{a}(\boldsymbol{x}, t)-\nabla_{\boldsymbol{x}} \cdot \boldsymbol{\sigma}(\boldsymbol{x}, t) & =\boldsymbol{f}(\boldsymbol{x}, t) \\
\nabla_{\boldsymbol{x}} \cdot \mathbb{d}(\boldsymbol{x}, t) & =0 \\
\boldsymbol{u}(\boldsymbol{x}, t) & =\overline{\boldsymbol{u}}(\boldsymbol{x}, t) \\
\boldsymbol{\sigma}(\boldsymbol{x}, t) \cdot \boldsymbol{n} & =\overline{\boldsymbol{t}}(\boldsymbol{x}, t) \\
\phi(\boldsymbol{x}, t) & =\bar{\phi}(\boldsymbol{x}, t) \\
-\mathbb{d} \cdot \boldsymbol{n} & =\bar{\omega} \\
\boldsymbol{u}(\boldsymbol{x}, 0) & =\boldsymbol{u}_{0}(\boldsymbol{x}) \\
\boldsymbol{v}(\boldsymbol{x}, 0) & =\boldsymbol{v}_{0}(\boldsymbol{x})
\end{aligned}
$$

$$
\begin{aligned}
& \forall \boldsymbol{x} \in \mathcal{B}_{t}, t \in\left[0, t_{f}\right] \\
& \forall \boldsymbol{x} \in \mathcal{B}_{t}, t \in\left[0, t_{f}\right] \\
& \forall \boldsymbol{x} \in \partial \mathcal{B}_{t}^{\text {mech,D }}, t \in\left[0, t_{f}\right] \\
& \forall \boldsymbol{x} \in \partial \mathcal{B}_{t}^{\text {mech, }}, t \in\left[0, t_{f}\right] \\
& \forall \boldsymbol{x} \in \partial \mathcal{B}_{t}^{\text {elec,D }}, t \in\left[0, t_{f}\right] \\
& \forall \boldsymbol{x} \in \partial \mathcal{B}_{t}^{\text {elec,N }}, t \in\left[0, t_{f}\right] \\
& \forall \boldsymbol{x} \in \mathcal{B}_{t} \\
& \forall \boldsymbol{x} \in \mathcal{B}_{t}
\end{aligned}
$$

where, $t$ is the time variable, $t_{f}$ is the final time, $\boldsymbol{v}$ is the velocity at point $\boldsymbol{x}, \boldsymbol{a}$ is the acceleration at point $\boldsymbol{x}, \boldsymbol{f}$ is the body force per unit deformed volume, $\boldsymbol{n}$ is the unit outward normal on the boundary $\partial \mathcal{B}_{t}, \overline{\boldsymbol{u}}$ is the prescribed value of displacement on the Dirichlet boundary $\partial \mathcal{B}_{t}^{\text {mech,D }}, \bar{t}$ is the specified traction force per unit deformed area on the Neumann boundary $\partial \mathcal{B}_{t}^{\text {mech,N }}, \bar{\phi}$ is the prescribed value of electric potential on the Dirichlet boundary $\partial \mathcal{B}_{t}^{\text {elec, } \mathrm{D}}, \bar{\omega}$ is the specified electric surface charge density per unit deformed area on the Neumann boundary $\partial \mathcal{B}_{t}^{\text {elec,N }}$, and $\boldsymbol{u}_{0}$ and $\boldsymbol{v}_{0}$ are the initial displacement and initial velocity, respectively. $\rho$ is the density of the solid in the current configuration, and it is related to the density of the solid in the original configuration, $\rho_{0}$, as

$$
\rho_{0}=\rho J
$$

\subsection{Modifications for incompressible materials}

For truly incompressible materials, the deformation of the solid is such that the total volume change at any point in the domain at any time instant is zero. This can be represented mathematically as the incompressibility constraint in finite strains,

$$
J=1 .
$$

For modelling incompressible materials in finite strains, the deformation gradient, $\boldsymbol{F}$, is decomposed into deviatoric and volumetric components as

$$
\boldsymbol{F}=\boldsymbol{F}_{\mathrm{vol}} \boldsymbol{F}_{\mathrm{dev}}
$$

with

$$
\boldsymbol{F}_{\mathrm{vol}}:=J^{1 / 3} \mathbf{I}, \quad \text { and } \quad \boldsymbol{F}_{\mathrm{dev}}:=J^{-1 / 3} \boldsymbol{F} .
$$

Using the above definitions, modified strain and stress measures are defined as

$$
\begin{aligned}
\text { Modified deformation gradient, } & \overline{\boldsymbol{F}}:=J^{-1 / 3} \boldsymbol{F} \\
\text { Modified right Cauchy-Green deformation tensor, } & \overline{\boldsymbol{C}}:=\overline{\boldsymbol{F}}^{\mathrm{T}} \overline{\boldsymbol{F}} \\
\text { Modified left Cauchy-Green deformation tensor, } & \overline{\boldsymbol{b}}:=\overline{\boldsymbol{F}} \overline{\boldsymbol{F}}^{\mathrm{T}} \\
\text { Modified Green-Lagrange strain tensor, } & \overline{\boldsymbol{E}}:=\frac{1}{2}[\overline{\boldsymbol{C}}-\boldsymbol{I}] \\
\text { Modified first Piola-Kirchhoff stress tensor, } & \overline{\boldsymbol{P}}:=\frac{\partial \Psi}{\partial \boldsymbol{F}} \\
\text { Modified second Piola-Kirchhoff stress tensor, } & \overline{\boldsymbol{S}}:=\frac{\partial \Psi}{\partial \boldsymbol{E}} \\
\text { Modified Cauchy stress tensor, } & \overline{\boldsymbol{\sigma}}:=\frac{1}{J} \overline{\boldsymbol{P}} \boldsymbol{F}
\end{aligned}
$$

Note that in (35) and (36), the energy function, $\Psi$, has to be modified accordingly in terms of modified strain measures. 


\section{Material models}

The material models in the present work are limited to incompressible isotropic hyperelastic materials with an electromechanical coupling. This, however, is not a limitation but chosen only for the clarity of the presentation. The proposed work offers straightforward extension to anisotropic as well as rate-dependent material models, for example, viscoelasticity by adding the corresponding energy contribution to the strain energy function. These extensions will be addressed in a forthcoming contribution.

For all of the material models considered in this work, the energy function is assumed to be decomposed into two parts as

$$
\Psi=\Psi^{\text {mech,dev }}+\Psi^{\text {coup }}
$$

where $\Psi^{\text {mech,dev }}$ is the deviatoric part of the mechanical strain energy function and $\Psi^{\text {coup }}$ is the strain energy function that accounts for the electromechanical coupling. It is important to mention at this point that the volumetric energy function $\Psi^{\mathrm{mech}, \mathrm{vol}}$ vanishes for the perfectly incompressible material models.

\subsection{Deviatoric energy function}

From a variety of deviatoric energy functions available in the literature for modelling rubber-like materials, we have selected three energy functions: Neo-Hookean, Gent and Arruda-Boyce models. For comprehensive details on energy functions suitable for rubber-like materials modelling, the reader is referred to review works of Steinmann et al. [75], Hossain and Steinmann [37], Hossain et al. [38], and Mihai and Goriely [135].

The energy functions for the three material models used in the present work are given as:

\section{- Neo-Hookean model}

$$
\Psi^{\text {mech,dev }}=\frac{\mu}{2}\left[I_{\bar{C}}-3\right]
$$

where $\mu$ is the shear modulus and $I_{\bar{C}}=\operatorname{tr}(\bar{C})$.

\section{- Gent model}

$$
\Psi^{\text {mech,dev }}=-\frac{\mu I_{m}}{2} \ln \left(1-\frac{I_{\bar{C}}-3}{I_{m}}\right)
$$

where $I_{m}$ is a material parameter that represents the upper limit of $\left[I_{\bar{C}}-3\right]$.

\section{- Arruda-Boyce model}

$$
\begin{aligned}
\Psi^{\text {mech,dev }} & =\frac{\mu}{2}\left[I_{\overline{\boldsymbol{C}}}-3\right]+\frac{\mu}{20 N}\left[I_{\overline{\boldsymbol{C}}}^{2}-3^{2}\right] \\
& +\frac{11 \mu}{1050 N^{2}}\left[I_{\overline{\boldsymbol{C}}}^{3}-3^{3}\right]+\frac{19 \mu}{7000 N^{3}}\left[I_{\bar{C}}^{4}-3^{4}\right]+\frac{519 \mu}{673750 N^{4}}\left[I_{\bar{C}}^{5}-3^{5}\right]
\end{aligned}
$$

where $N$ is the measure of cross-link density of polymers.

\subsection{Coupled energy function}

The part of the strain energy function corresponding to the electromechanical coupling is taken similar to the one proposed in Hennan et al. [32], Bishara and Jabareen [7, 40], Mehnert et al. [56],

$$
\Psi^{\text {coup }}=-\frac{1}{2} \varepsilon J C^{-1}:[\mathbb{E} \otimes \mathbb{E}]=-\frac{1}{2} \varepsilon J \mathbb{E}_{I} C_{I J}^{-1} \mathbb{E}_{J},
$$

where $\varepsilon=\varepsilon_{r} \varepsilon_{0}$ is the permittivity of the material and $\varepsilon_{r}$ is the relative permittivity, also know as the dielectric constant. Note that there are experimental evidences that the dielectric constant depends on the initial stretching of EAPs [40]. However, for simplicity, in this work, we have taken the dielectric parameter $\varepsilon$ as a constant. 


\section{Displacement-pressure-potential finite element formulation in finite strains}

The selective reduced integration approach [124], and the $\boldsymbol{F}$-bar, $J$-bar and three-field displacementpressure-Jacobian formulations for the mechanical problem enforce the incompressibility constraint only approximately by using a volumetric energy function and choosing a large value of the bulk modulus $\kappa$. However, since the volumetric energy function vanishes for the truly incompressible case, these formulations are no longer suitable, and the pressure field must be determined as an additional solution variable. Mathematically, $p$ is a Lagrange multiplier for enforcing the incompressibility constraint given in the equation (28).

Moreover, while the $\boldsymbol{F}$-bar formulation has proven to be effective for modelling nearly incompressible hyperelastic materials, the fundamental issue with the formulation is its computational cost, especially when the Poisson's ratio approaches 0.5 . Because of the direct dependence of the elasticity tensor on the bulk modulus similar to the pure displacement formulation, the $\boldsymbol{F}$-bar still requires as many load steps as necessary for the displacement formulation. As demonstrated in the section of numerical examples, for the case of $\kappa / \mu=$ $10^{5}$, the $\boldsymbol{F}$-bar formulation requires 100 times more load steps when compared with the three-field and the proposed mixed formulations. Our experience indicates that this number increases further as the Poisson's ratio approaches 0.5 , i.e., for $\kappa / \mu>10^{5}$. The same arguments extend to the recently proposed $J$-bar formulation because it still retains the volumetric contribution in the elasticity tensor. Although the three-field formulation for the purely mechanical cases has proven to be effective in dealing with volumetric locking, because of its failure to satisfy the inf-sup stability condition, the Q1/P0 element produces pressure fields with spurious oscillations, otherwise known as checkerboard pattern in the literature. The Q1/P0 element also suffers from convergence issues for significantly higher values of the bulk modulus, as will be demonstrated in Section 5.

It has been proven in Kadapa [107] that a two-field mixed displacement-pressure formulation for mechanical problem is sufficient enough for computing accurate numerical solutions of incompressible materials. Following Kadapa [107, 112] and Kadapa et al. [108], the two-field mixed displacement-pressure formulation will be extended to electromechanics in this work. Note that the classical three-field formulation becomes a four-field (displacement-pressure-Jacobian-electric potential) formulation in the case of electromechanical problems while our two-field formulation will become a three-field (displacement-pressure-electric potential) one for electromechanics.

Following the Hamilton's principle $[130,131,132,133]$, the action functional, $\mathcal{A}$, for the displacementpressure-electric potential $(\boldsymbol{u} / p / \phi)$ formulation can be written as

$$
\mathcal{A}=\int_{0}^{t_{f}} \mathcal{L}(\boldsymbol{u}, \boldsymbol{v}, p, \phi, t) d t
$$

where $\mathcal{L}$ is the Lagrangian which, in terms of total kinetic energy, $\mathcal{T}$, and total potential energy, $\mathcal{V}$, of the system is given as

$$
\mathcal{L}=\underbrace{\int_{\mathcal{B}_{0}} \frac{1}{2} \rho_{0} \boldsymbol{v}^{2} \mathrm{~d} V}_{\mathcal{T}}-\underbrace{\left[\int_{\mathcal{B}_{0}} \Psi(\overline{\boldsymbol{C}}, p, \phi) \mathrm{d} V+\int_{\mathcal{B}_{0}} \Psi_{\mathrm{PL}} \mathrm{d} V-\mathcal{V}_{\text {ext }}\right]}_{\mathcal{V}}
$$

where $\mathrm{d} V$ is the elemental volume in the reference configuration; $\Psi_{\mathrm{PL}}$ is the energy function for imposing the incompressibility constraint (28) using the perturbed Lagrangian method with $p$ as the Lagrange multiplier; and $\mathcal{V}_{\text {ext }}$ is the energy contribution due to the external forces. $\Psi_{\mathrm{PL}}$ is given as

$$
\Psi_{\mathrm{PL}}=p[J-1]-\frac{p^{2}}{2 \kappa}
$$

where $\kappa$ is the bulk modulus of the material. The term $-\frac{p^{2}}{2 \kappa}$ in equation (45) accounts for the nearly incompressible case, i.e. when $\nu \approx 0.5$, in the proposed framework. The energy contribution due to the external forces is given by

$$
\mathcal{V}_{\text {ext }}=\int_{\mathcal{B}_{0}} \boldsymbol{u}^{\mathrm{T}} \boldsymbol{f}_{0} \mathrm{~d} V+\int_{\partial \mathcal{B}_{0}^{\text {mech }, \mathrm{N}}} \boldsymbol{u}^{\mathrm{T}} \boldsymbol{t}_{0} \mathrm{~d} A+\int_{\partial \mathcal{B}_{0}^{\text {elec, } \mathrm{N}}} \phi \omega_{0} \mathrm{~d} A .
$$

where $\mathrm{d} A$ is the elemental area in the reference configuration. 
Now, using the principle of stationary action, i.e., $\delta \mathcal{A}=0$, and then applying the integration by parts for the term due to kinetic energy, we obtain

$$
\delta \mathcal{A}=\int_{\mathcal{B}_{0}} \delta u_{i} \rho_{0} a_{i} \mathrm{~d} V+\int_{\mathcal{B}_{0}}\left[\delta u_{i, j} \widehat{\sigma}_{i j}+\delta \phi_{, i} \mathbb{d}_{i}\right] J \mathrm{~d} V+\int_{\mathcal{B}_{0}} \delta p\left[J-1-\frac{p}{\kappa}\right] \mathrm{d} V-\delta \mathcal{V}_{\text {ext }}=0
$$

where $\delta(\bullet)$ denotes the variation of the variable $(\bullet)$ and $(\circ)_{, j}$ denotes the derivative of $(\circ)$ with respect to the $j$ th component; $a_{i}$ is the $i$ th component of acceleration $\boldsymbol{a}$ and $\widehat{\sigma}_{i j}$ is the effective Cauchy stress tensor for the mixed formulation. We refer the reader to Appendix A. for the details on the variations of energy functions.

For the finite element discretisation, we take the approximations for displacement $(\boldsymbol{u})$, pressure $(p)$ and electric potential $(\phi)$ as

$$
\boldsymbol{u}=\mathbf{N}_{\boldsymbol{u}} \mathbf{u}, \quad p=\mathbf{N}_{p} \mathbf{p}, \quad \text { and } \quad \phi=\mathbf{N}_{\phi} \phi,
$$

where $\mathbf{u}, \mathbf{p}$ and $\phi$ are the displacement, pressure and electric potential degrees of freedom, and

$$
\begin{gathered}
\mathbf{N}_{\boldsymbol{u}}=\left[\begin{array}{ccccccc}
{ }^{1} N_{\boldsymbol{u}} & 0 & 0 & \ldots & { }^{\mathrm{nu}} N_{\boldsymbol{u}} & 0 & 0 \\
0 & { }^{1} N_{\boldsymbol{u}} & 0 & \ldots & 0 & { }^{\mathrm{nu}} N_{\boldsymbol{u}} & 0 \\
0 & 0 & { }^{1} N_{\boldsymbol{u}} & \ldots & 0 & 0 & { }^{\mathrm{nu}} N_{\boldsymbol{u}}
\end{array}\right] \\
\mathbf{N}_{p}=\left[\begin{array}{lllll}
{ }^{1} N_{p} & { }^{2} N_{p} & \ldots & { }^{\mathrm{np}} N_{p}
\end{array}\right] \\
\mathbf{N}_{\phi}=\left[\begin{array}{lllll}
{ }^{1} N_{\phi} & { }^{2} N_{\phi} & \ldots & { }^{\mathrm{n} \phi} N_{\phi}
\end{array}\right]
\end{gathered}
$$

with nu, np and $\mathrm{n} \phi$ are the number of the basis functions, respectively, for the displacement, pressure and electric potential degrees of freedom for an element. After substituting the variations of the field variables

$$
\delta \boldsymbol{u}=\mathbf{N}_{\boldsymbol{u}} \delta \mathbf{u}, \quad \delta p=\mathbf{N}_{p} \delta \mathbf{p}, \quad \text { and } \quad \delta \phi=\mathbf{N}_{\phi} \delta \phi,
$$

into the first variation given in equation (47), the resulting semi-discrete equations for the mixed formulation can be written as

$$
\begin{aligned}
& \mathbf{F}_{\boldsymbol{u}}^{\text {int }}=\mathbf{F}_{\boldsymbol{u}}^{\mathrm{ext}}, \\
& \mathbf{F}_{p}^{\text {int }}=\mathbf{0}, \\
& \mathbf{F}_{\phi}^{\text {int }}=\mathbf{F}_{\phi}^{\text {ext }},
\end{aligned}
$$

where $\mathbf{F}_{\boldsymbol{u}}^{\text {int }}, \mathbf{F}_{p}^{\text {int }}$ and $\mathbf{F}_{\phi}^{\text {int }}$ are the vectors of internal forces for the displacement, pressure and potential fields, respectively; and $\mathbf{F}_{\boldsymbol{u}}^{\mathrm{ext}}$ and $\mathbf{F}_{\phi}^{\mathrm{ext}}$ are the vectors of external forces for the displacement and potential fields, respectively. These force vectors are given by

$$
\begin{aligned}
\mathbf{F}_{\boldsymbol{u}}^{\mathrm{int}} & =\int_{\mathcal{B}_{0}} \mathbf{N}_{\boldsymbol{u}}^{\mathrm{T}}\left[\rho_{0} \boldsymbol{a}\right] \mathrm{d} V+\int_{\mathcal{B}_{t}} \mathbf{G}_{\boldsymbol{u}}^{\mathrm{T}} \widehat{\boldsymbol{\sigma}} \mathrm{d} v, \\
\mathbf{F}_{\boldsymbol{u}}^{\text {ext }} & =\int_{\mathcal{B}_{0}} \mathbf{N}_{\boldsymbol{u}}^{\mathrm{T}} \boldsymbol{f}_{0} \mathrm{~d} V+\int_{\partial \mathcal{B}_{0}^{\text {mech, N }}} \mathbf{N}_{\boldsymbol{u}}^{\mathrm{T}} \boldsymbol{t}_{0} \mathrm{~d} A, \\
\mathbf{F}_{p}^{\mathrm{int}} & =\int_{\mathcal{B}_{0}} \mathbf{N}_{p}^{\mathrm{T}}\left[J-1-\frac{p}{\kappa}\right] \mathrm{d} V, \\
\mathbf{F}_{\phi}^{\mathrm{int}} & =\int_{\mathcal{B}_{t}} \mathbf{G}_{\phi}^{\mathrm{T}} \mathrm{d} \mathrm{d} v, \\
\mathbf{F}_{\phi}^{\text {ext }} & =\int_{\partial \mathcal{B}_{0}^{\text {elec, N }}} \mathbf{N}_{\phi}^{\mathrm{T}} \omega_{0} \mathrm{~d} A .
\end{aligned}
$$


where $\mathrm{d} v$ is the elemental volume in the current configuration. $\mathbf{G}_{\boldsymbol{u}}$ and $\mathbf{G}_{\phi}$ are the gradient operators for the displacement and potential fields, respectively. For more details on the derivations of these quantities, see Appendix B.

\subsection{Fully-implicit time integration}

We solve the coupled nonlinear equations in (53) using the monolithic approach and use the state-of-the-art implicit time integration scheme proposed by Kadapa et al. [109] for marching in time. The advantage of the implicit scheme of [109] over the widely-used Newmark- $\beta$ scheme [125] lies in its ability to damp out high-frequency modes using a user-controlled parameter, see [136]. The numerical solutions of the coupled nonlinear equations are computed iteratively using the Newton-Raphson scheme. To achieve linearisations for the Newton-Rapshon scheme, we apply the second variation to the action functional, $\mathcal{A}$, as presented in Appendix A.

By adapting the implicit time integration scheme of [109] and substituting the corresponding finite element approximations for three field variables, we obtain the following discrete matrix system for the incremental displacements, $\Delta \mathbf{u}$, incremental pressure, $\Delta \mathbf{p}$, and incremental electric potential, $\Delta \phi$, at iteration $k+1$ as

$$
\left[\begin{array}{ccc}
\mathbf{K}_{u \boldsymbol{u}} & \mathbf{K}_{\boldsymbol{u} p} & \mathbf{K}_{\boldsymbol{u} \phi} \\
\mathbf{K}_{p \boldsymbol{u}} & \mathbf{K}_{p p} & \mathbf{0} \\
\mathbf{K}_{\phi \boldsymbol{u}} & \mathbf{0} & \mathbf{K}_{\phi \phi}
\end{array}\right]\left\{\begin{array}{c}
\Delta \mathbf{u} \\
\Delta \mathbf{p} \\
\Delta \boldsymbol{\phi}
\end{array}\right\}=-\left\{\begin{array}{l}
\mathbf{R}_{\boldsymbol{u}} \\
\mathbf{R}_{p} \\
\mathbf{R}_{\phi}
\end{array}\right\}
$$

In the above system of matrix, individual components can be obtained as

$$
\begin{aligned}
\mathbf{K}_{\boldsymbol{u} \boldsymbol{u}} & =\frac{\alpha_{m}^{2}}{\alpha_{f} \gamma^{2} \Delta t^{2}} \mathbf{M}_{\boldsymbol{u} \boldsymbol{u}}+\alpha_{f} \int_{\mathcal{B}_{t}} \mathbf{G}_{\boldsymbol{u}}^{\mathrm{T}} \mathbf{e} \mathbf{G}_{\boldsymbol{u}} \mathrm{d} v \\
\mathbf{M}_{\boldsymbol{u} \boldsymbol{u}} & =\int_{\mathcal{B}_{0}} \rho_{0} \mathbf{N}_{\boldsymbol{u}}^{\mathrm{T}} \mathbf{N}_{\boldsymbol{u}} \mathrm{d} V \\
\mathbf{K}_{\boldsymbol{u} p} & =\alpha_{f} \int_{\mathcal{B}_{t}} \mathbf{D}_{\boldsymbol{u}}^{\mathrm{T}} \mathbf{N}_{p} \mathrm{~d} v=\mathbf{K}_{p \boldsymbol{u}}^{\mathrm{T}} \\
\mathbf{K}_{\boldsymbol{u} \phi} & =\alpha_{f} \int_{\mathcal{B}_{t}} \mathbf{G}_{\boldsymbol{u}}^{\mathrm{T}} \mathbf{p} \mathbf{G}_{\phi} \mathrm{d} v=\mathbf{K}_{\phi \boldsymbol{u}}^{\mathrm{T}} \\
\mathbf{K}_{p p} & =-\alpha_{f} \int_{\mathcal{B}_{0}} \frac{1}{\kappa} \mathbf{N}_{p}^{\mathrm{T}} \mathbf{N}_{p} \mathrm{~d} V \\
\mathbf{K}_{\phi \phi} & =\alpha_{f} \int_{\mathcal{B}_{t}} \mathbf{G}_{\phi}^{\mathrm{T}} \mathbf{d} \mathbf{G}_{\phi} \mathrm{d} v \\
\mathbf{R}_{\boldsymbol{u}} & =\mathbf{F}_{\boldsymbol{u}}^{\mathrm{int}}\left(\boldsymbol{a}_{n+\alpha_{m}}^{k}, \boldsymbol{u}_{n+\alpha_{f}}^{k}, p_{n+\alpha_{f}}^{k}, \phi_{n+\alpha_{f}}^{k}\right)-\mathbf{F}_{\boldsymbol{u}, n+\alpha_{f}}^{\mathrm{ext}}, \\
\mathbf{R}_{p} & =\mathbf{F}_{p}^{\mathrm{int}}\left(\boldsymbol{u}_{n+\alpha_{f}}^{k}, p_{n+\alpha_{f}}^{k}\right) \\
\mathbf{R}_{\phi} & =\mathbf{F}_{\phi}^{\mathrm{int}}\left(\boldsymbol{u}_{n+\alpha_{f}}^{k}, p_{n+\alpha_{f}}^{k}, \phi_{n+\alpha_{f}}^{k}\right)-\mathbf{F}_{\phi, n+\alpha_{f}}^{\mathrm{ext}}
\end{aligned}
$$




$$
\begin{aligned}
\mathbf{F}_{\boldsymbol{u}, n+\alpha_{f}}^{\mathrm{ext}} & =\alpha_{f} \mathbf{F}_{\boldsymbol{u}, n+1}^{\mathrm{ext}}+\left[1-\alpha_{f}\right] \mathbf{F}_{\boldsymbol{u}, n}^{\mathrm{ext}} \\
\mathbf{F}_{\phi, n+\alpha_{f}}^{\mathrm{ext}} & =\alpha_{f} \mathbf{F}_{\phi, n+1}^{\mathrm{ext}}+\left[1-\alpha_{f}\right] \mathbf{F}_{\phi, n}^{\mathrm{ext}} \\
\mathbf{a}_{n+\alpha_{m}}^{k} & =\alpha_{m} \mathbf{a}_{n+1}^{k}+\left[1-\alpha_{m}\right] \mathbf{a}_{n} \\
\mathbf{v}_{n+\alpha_{f}}^{k} & =\alpha_{f} \mathbf{v}_{n+1}^{k}+\left[1-\alpha_{f}\right] \mathbf{v}_{n} \\
\mathbf{u}_{n+\alpha_{f}}^{k} & =\alpha_{f} \mathbf{u}_{n+1}^{k}+\left[1-\alpha_{f}\right] \mathbf{u}_{n} \\
\mathbf{p}_{n+\alpha_{f}}^{k} & =\alpha_{f} \mathbf{p}_{n+1}^{k}+\left[1-\alpha_{f}\right] \mathbf{p}_{n} \\
\boldsymbol{\phi}_{n+\alpha_{f}}^{k} & =\alpha_{f} \boldsymbol{\phi}_{n+1}^{k}+\left[1-\alpha_{f}\right] \boldsymbol{\phi}_{n} \\
\mathbf{v}_{n+1}^{k} & =\frac{\alpha_{m}}{\alpha_{f} \gamma \Delta t}\left[\mathbf{u}_{n+1}^{k}-\mathbf{u}_{n}\right]+\frac{\left[\alpha_{f}-1\right]}{\alpha_{f}} \mathbf{v}_{n}+\frac{\left[\gamma-\alpha_{m}\right]}{\gamma \alpha_{f}} \dot{\mathbf{u}}_{n} \\
\mathbf{a}_{n+1}^{k} & =\frac{\alpha_{m}}{\alpha_{f} \gamma^{2} \Delta t^{2}}\left[\mathbf{u}_{n+1}^{k}-\mathbf{u}_{n}\right]-\frac{1}{\alpha_{f} \gamma \Delta t} \mathbf{v}_{n}+\frac{\gamma-1}{\gamma} \mathbf{a}_{n}+\frac{\left[\gamma-\alpha_{m}\right]}{\alpha_{f} \gamma^{2} \Delta t} \dot{\mathbf{u}}_{n} \\
\dot{\mathbf{u}}_{n+1}^{k} & =\frac{1}{\gamma \Delta t}\left[\mathbf{u}_{n+1}^{k}-\mathbf{u}_{n}\right]+\frac{\gamma-1}{\gamma} \dot{\mathbf{u}}_{n}
\end{aligned}
$$

where $\Delta t$ is the time step, $\mathbf{D}_{\boldsymbol{u}}$ is the divergence operator, see Appendix B; $\mathbf{u}_{n+1}^{k}, \mathbf{v}_{n+1}^{k}, \mathbf{a}_{n+1}^{k}, \mathbf{p}_{n+1}^{k}$ and $\phi_{n+1}^{k}$ are the displacement, velocity, acceleration, pressure, and electric potential, respectively, at the $k^{t h}$ iteration of the Newton-Raphson scheme; $\dot{\mathbf{u}}$ is the time derivative of the displacement which is different from velocity, $\mathbf{v}$; see [109]. Note that $\mathbf{e}, \mathbf{p}$ and $\mathbf{d}$, respectively, in (60), (63) and (65) are the corresponding matrix versions of the tensors $\mathrm{e}_{i j k l}, \mathrm{p}_{i j k}, \mathrm{~d}_{i j}$. Following the matrix/vector format used for the gradient and stress tensors in Appendix B, matrix forms of $\mathbf{e}, \mathbf{p}$ and $\mathbf{d}$ are of size $9 \times 9,9 \times 3$ and $3 \times 3$, respectively.

Following Kadapa et al. [109], the time integration scheme is second-order accurate and unconditionallystable when the parameters are chosen such that

$$
\alpha_{f}=\frac{1}{1+\rho_{\infty}} ; \quad \alpha_{m}=\frac{3-\rho_{\infty}}{2\left[1+\rho_{\infty}\right]} ; \quad \gamma=\frac{1}{2}+\alpha_{m}-\alpha_{f}, \quad \text { for } \quad 0 \leq \rho_{\infty} \leq 1,
$$

where $\rho_{\infty}$ is the spectral radius at an infinite time step and it controls the amount of numerical damping. For $\rho_{\infty}=1.0$, the scheme does not include any numerical damping and for $\rho_{\infty}=0.0$, it annihilates all the highfrequency modes after the first time step. For comprehensive details on the implicit time integration scheme, the reader is referred to Kadapa et al. [109].

Remark 1: The second term in equation (60) for the effective stiffness matrix $\mathbf{K}_{u u}$ accounts for both the material and geometric stiffness terms.

Remark 2: The formulation for the elastostatics problems is recovered by simply setting the parameters as, $\alpha_{f}=1$ and $\alpha_{m}=0$.

Remark 3: For the four-field (Q1/P0) and $\boldsymbol{F}$-bar formulations, the volumetric energy function is taken as

$$
\Psi^{\mathrm{mech}, \mathrm{vol}}=\frac{\kappa}{2}[J-1]^{2}
$$

It is easy to show that equation (54) in the proposed formulation exactly recovers the relation

$$
p=\frac{\partial \Psi^{\mathrm{mech}, \mathrm{vol}}}{\partial J}=\kappa[J-1],
$$

for the nearly incompressible case. Note that there are several other forms of volumetric strain energy functions, see Holzapfel [134], and they can be incorporated into the proposed framework as already demonstrated in Kadapa [112].

\subsection{Finite element spaces for the mixed formulation}

From the various finite element spaces available in the literature for the mixed displacement-pressure formulation in solid mechanics problems, we can choose either the P2/P1 and P2/P0 elements [110, 116] or stabilised formulations $[118,119,120]$ that are based on the Lagrange family of elements or NURBS based elements [107, 108] or the BT2/BT1 and BT2/BT0 elements that are based on Bézier family of elements [112]. 
Although P2/P1, Q2/Q1 and BT2/BT1 elements have been used widely for incompressible solids mechanics problems, the adaption of the $\mathrm{Q} 2 / \mathrm{P} 1$ element to solid mechanics problems is scarce when compared to its usage for incompressible fluid flow problems. To the best of the authors' knowledge, extensive studies on the adaptation of these elements to coupled electromechanics are either sparse or non-existent; literature on computational electromechanics is predominantly restricted to the Q1/P0 and Q1-F-bar elements [32, 46, 60, 67, 74, 90, 123]. Thanks to the advantages of Bézier elements over Lagrange elements for explicit dynamics simulations and their ease of mesh generation over NURBS [111, 112], our primary choice of finite elements in this work is the one based on Bézier elements. However, for the sake of completeness, Lagrange family of elements are also considered.

We name the new elements as BT2/BT1/BT2, P2/P1/P2, Q2/P1/Q2 and Q2/Q1/Q2 for the three-field mixed displacement-pressure-electric potential $(\boldsymbol{u} / p / \phi)$ formulation. For the BT2/BT1/BT2 and P2/P1/P2 elements, $\mathrm{nu}=\mathrm{n} \phi=6$ and $\mathrm{np}=3$ in 2D, and $\mathrm{nu}=\mathrm{n} \phi=10$ and $\mathrm{np}=4$ in $3 \mathrm{D}$; for the Q2/P1/Q2 element, $\mathrm{nu}=\mathrm{n} \phi=9$ and $\mathrm{np}=3$ in $2 \mathrm{D}$, and $\mathrm{nu}=\mathrm{n} \phi=27$ and $\mathrm{np}=4$ in $3 \mathrm{D}$; and for the Q2/Q1/Q2 element, $\mathrm{nu}=\mathrm{n} \phi=9$ and $\mathrm{np}=4$ in $2 \mathrm{D}$, and $\mathrm{nu}=\mathrm{n} \phi=27$ and $\mathrm{np}=8$ in $3 \mathrm{D}$.

The computational advantages of the proposed elements over the widely-used Q1/P0 element for computational electromechanics can be summarised:

1. The order of convergence for the displacement, pressure and stress fields is one order higher for the proposed elements when compared with the Q1/P0 element. Therefore, accurate numerical results can be obtained using coarse meshes with the proposed elements, as will be demonstrated with few numerical examples in Sections 5 and 6.

2. The proposed elements are inf-sup stable in both two and three dimensions [112] while the Q1/P0 element is not, see [113]. Therefore, the proposed element produces pressure fields that are free from spurious modes [112] as opposed to the Q1/P0 element which yields spurious modes in the pressure field [104, 117].

3. The task of mesh generation for the Q1 and Q2 elements is quite cumbersome for complex geometries. However, automated tetrahedral mesh generation tools can be used for discretising complex geometries with the BT2 and P2 elements.

4. While it is true that the pressure degrees of freedom (DOFs) in the proposed work increase the size of coupled matrix system that needs to be solved at each iteration, it can be shown that the number of pressure DOFs for the BT2/BT1/BT2, $\mathrm{P} 2 / \mathrm{P} 1 / \mathrm{P} 2$ and $\mathrm{Q} 2 / \mathrm{Q} 1 / \mathrm{Q} 2$ elements is only about $5 \%$ of the combined DOFs for the displacement and electrical potential. This is illustrated schematically in Fig. 1a and using a matrix pattern in Fig. 1b for the example of a spherical gripper presented in Section 5.4. Moreover, the convergence of Newton-Raphson iterations for the proposed elements is not sensitive to the changes in the values of the bulk modulus.

On the contrary, although the pressure DOFs can be condensed out for the Q1/P0 element when used for the nearly incompressible materials, such a reduction is not always computationally beneficial. To demonstrate this, the coupled matrix system for the Q1/P0 element [67]

$$
\left[\begin{array}{cccc}
\mathbf{K}_{\boldsymbol{u} \boldsymbol{u}} & \mathbf{0} & \mathbf{K}_{\boldsymbol{u} p} & \mathbf{K}_{\boldsymbol{u} \phi} \\
\mathbf{0} & \mathbf{K}_{\tilde{J} \tilde{J}} & \mathbf{K}_{\tilde{J} p} & \mathbf{0} \\
\mathbf{K}_{p \boldsymbol{u}} & \mathbf{K}_{p \tilde{J}} & \mathbf{0} & \mathbf{0} \\
\mathbf{K}_{\phi \boldsymbol{u}} & \mathbf{0} & \mathbf{0} & \mathbf{K}_{\phi \phi}
\end{array}\right]\left\{\begin{array}{c}
\Delta \mathbf{u} \\
\Delta \tilde{\boldsymbol{J}} \\
\Delta \mathbf{p} \\
\Delta \boldsymbol{\phi}
\end{array}\right\}=-\left\{\begin{array}{l}
\mathbf{R}_{\boldsymbol{u}} \\
\mathbf{R}_{\tilde{J}} \\
\mathbf{R}_{p} \\
\mathbf{R}_{\phi}
\end{array}\right\}
$$

is rewritten in a condensed form as

$$
\left[\begin{array}{ll}
\widetilde{\mathbf{K}}_{u u} & \mathbf{K}_{u \phi} \\
\mathbf{K}_{\phi \boldsymbol{u}} & \mathbf{K}_{\phi \phi}
\end{array}\right]\left\{\begin{array}{c}
\Delta \mathbf{u} \\
\Delta \phi
\end{array}\right\}=-\left\{\begin{array}{l}
\widetilde{\mathbf{R}}_{u} \\
\mathbf{R}_{\phi}
\end{array}\right\}
$$


where $\tilde{J}$ is the independent approximation for the Jacobian and

$$
\begin{aligned}
\widetilde{\mathbf{K}}_{\boldsymbol{u u}} & =\mathbf{K}_{\boldsymbol{u u}}+\mathbf{K}_{\boldsymbol{u} p} \mathbf{K}_{\tilde{J} p}^{-1} \mathbf{K}_{\tilde{J} \tilde{J}} \mathbf{K}_{p \tilde{J}}^{-1} \mathbf{K}_{p \boldsymbol{u}} \\
\widetilde{\mathbf{R}}_{\boldsymbol{u}} & =\mathbf{R}_{\boldsymbol{u}}-\mathbf{K}_{u p} \mathbf{K}_{\tilde{J} p}^{-1} \mathbf{R}_{\tilde{J}}+\mathbf{K}_{\boldsymbol{u} p} \mathbf{K}_{\tilde{J} p}^{-1} \mathbf{K}_{\tilde{J} \tilde{J}} \mathbf{K}_{p \tilde{J}}^{-1} \mathbf{R}_{p} .
\end{aligned}
$$

By exploiting the constant discontinuous approximation for the pressure and Jacobian variables, $\widetilde{\mathbf{K}}_{u \boldsymbol{u}}$ and $\widetilde{\mathbf{R}}_{u}$ for the volumetric energy function (80) can be further simplified as

$$
\begin{aligned}
\widetilde{\mathbf{K}}_{u \boldsymbol{u}} & =\mathbf{K}_{u \boldsymbol{u}}+\frac{\kappa}{V_{0}} \mathbf{K}_{\boldsymbol{u} p} \mathbf{K}_{p u} \\
\widetilde{\mathbf{R}}_{\boldsymbol{u}} & =\mathbf{R}_{\boldsymbol{u}}+\frac{1}{V_{0}} \mathbf{K}_{u p}\left[\kappa \mathbf{R}_{p}-\mathbf{R}_{\tilde{J}}\right],
\end{aligned}
$$

where $V_{0}$ is the volume of the element in the reference configuration. It can be observed from (86) and (87) that both $\widetilde{\mathbf{K}}_{u u}$ and $\widetilde{\mathbf{R}}_{u}$ depend directly on $\kappa$, the bulk modulus. Such a dependence on the bulk modulus significantly affects the convergence of iterations in the Newton-Raphson scheme for $\kappa / \mu>$ $10^{3}$, often causing the convergence to stall and eventually crashing the simulation, as demonstrated with the example of a spherical gripper in Section 5.4. Besides, such a reduction is not applicable to truly incompressible material models. Note that the convergence issues associated with the Q1/P0 element are also applicable to the $\mathrm{Q} 2 / \mathrm{P} 1 / \mathrm{Q} 2$ element when the pressure DOFs are eliminated from the global matrix system. However, Q2/P1/Q2 element is not the primary focus of the present work.

Therefore, the additional cost of matrix solver for computing the pressure DOFs is insignificant when compared with the advantages gained: (i) increased accuracy in the stresses, (ii) the ability to simulate truly incompressible materials, (iii) robustness in achieving the convergence, and (iv) the ease of mesh generation for complex geometries. Thus, the proposed work introduces a new finite element framework that offers many advantages over the Q1/P0 and Q1-F-bar elements, as tabulated in Table 1, for computing the numerical solutions of coupled electromechanical problems.

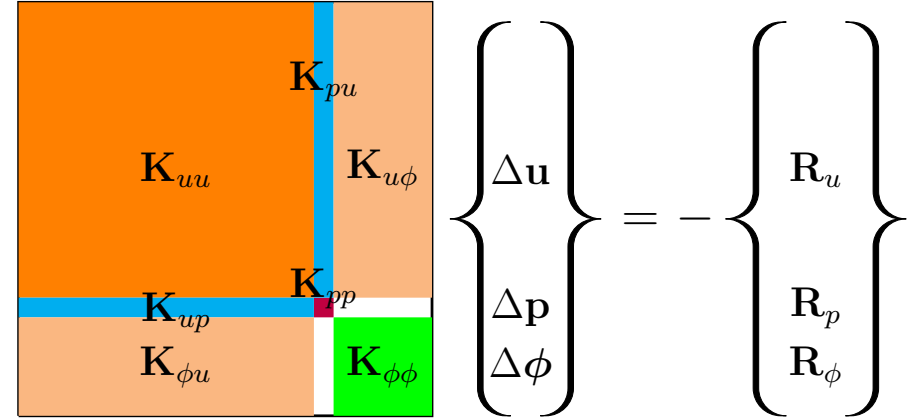

(a)

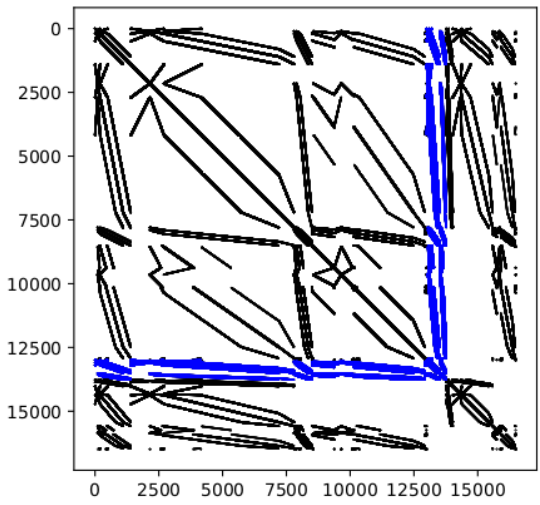

(b)

Figure 1: (a) Schematic depiction of the global matrix system for the BT2/BT1/BT2, P2/P1/P2 and Q2/Q1/Q2 elements and (b) sparse matrix pattern for the BT2/BT1/BT2 and P2/P1/P2 elements for the mesh in Fig. 11c for the spherical gripper example. Blue dots represent the entries corresponding to the $\mathbf{K}_{\boldsymbol{u} p}, \mathbf{K}_{p \boldsymbol{u}}$ and $\mathbf{K}_{p p}$ matrices. The number of displacement and potential DOFs for this test case are 13000 and 2727, respectively, and the number of pressure DOFs is 765 , which is only $4.86 \%$ of the combined displacement and electric potential DOFs. 


\begin{tabular}{|l|c|c|c|c|c|}
\hline & $\begin{array}{c}\text { Q1/P0 } \\
\text { Q1-F-bar }\end{array}$ & Q2/P1/Q2 & Q2/Q1/Q2 & P2/P1/P2 & BT2/BT1/BT2 \\
\hline Inf-sup stability & No & Yes & Yes & Yes & Yes \\
Accuracy in displacement & $\mathcal{O}\left(h^{2}\right)$ & $\mathcal{O}\left(h^{3}\right)$ & $\mathcal{O}\left(h^{3}\right)$ & $\mathcal{O}\left(h^{3}\right)$ & $\mathcal{O}\left(h^{3}\right)$ \\
Accuracy in potential & $\mathcal{O}\left(h^{2}\right)$ & $\mathcal{O}\left(h^{3}\right)$ & $\mathcal{O}\left(h^{3}\right)$ & $\mathcal{O}\left(h^{3}\right)$ & $\mathcal{O}\left(h^{3}\right)$ \\
Accuracy in stresses & $\mathcal{O}(h)$ & $\mathcal{O}\left(h^{2}\right)$ & $\mathcal{O}\left(h^{2}\right)$ & $\mathcal{O}\left(h^{2}\right)$ & $\mathcal{O}\left(h^{2}\right)$ \\
Truly incompressible models & No & No & Yes & Yes & Yes \\
Ease of mesh generation & No & No & No & Yes & Yes \\
Lumped masses & Yes & Yes & Yes & No & Yes \\
\hline
\end{tabular}

Table 1: Comparison of some salient features of different element types considered in this work.

\section{Numerical examples: electro-elastostatics}

To assess the accuracy and computational advantages of the proposed finite element framework and to demonstrate its effectiveness as a promising numerical framework for the simulation of EAPs, we consider several numerical examples in electro-elastostatics and electro-elastodynamics. These examples involve problems with the analytical solution, simple bending behaviour in actuators, buckling instabilities in thin polymeric shells and frequency-dependent external excitations. The accuracy and computational efficiency of the proposed framework are demonstrated first using elastostatic examples in this section and then using elastodynamics examples in the following section.

The proposed framework is implemented in an in-house computer code using the object-oriented programming concepts in $\mathrm{C}++$ and the Eigen library [126] for dense and sparse matrix operations. The coupled matrix systems are solved using the sparse parallel direct solver PARDISO [128, 129]. The finite element meshes used in the present work are generated using HyperMesh [127] and GMSH [138].

Units: Due to substantial variations in the magnitude of material parameters between the mechanical and electrical fields, monolithic schemes for coupled electromechanical problems modelled in the SI units leads to matrix systems whose non-zero coefficients vary by 14-20 orders of magnitude, leading to numerical truncation errors beyond the machine precision. Such matrix systems pose serious challenges in obtaining numerical solutions. To overcome such numerical issues, we propose the practise of modelling with the units for the basic quantities as tabulated in Table 2. Using the units in Table 2, some important quantities such as permittivity of the free space and the shear modulus become $\varepsilon_{0}=8.854 \times 10^{-12} \frac{\mathrm{F}}{\mathrm{m}}=8.854 \frac{\mathrm{g} \mathrm{mm}}{\mathrm{kV}^{2} \mathrm{~s}^{2}}$ and $\mu=(\bullet) \mathrm{Pa}=$ $(\bullet) \frac{\mathrm{g}}{\mathrm{mms}^{2}}$, respectively. Thus, while the numerical value of the shear modulus remains the same, the value of the permittivity of the free space is increased by $10^{12}$, bringing its numerical value to within the range of the material parameters of the mechanical problem. This results in matrix systems that are easier to solve when compared with those resulting from the use of base SI units.

\begin{tabular}{|l|l|}
\hline Variables & Units \\
\hline Length & millimeters $(\mathrm{mm})$ \\
Mass & grams $(\mathrm{g})$ \\
Time & seconds $(\mathrm{s})$ \\
Electric potential & kilo Volts $(\mathrm{kV})$ \\
\hline
\end{tabular}

Table 2: Units used in the present work for fundamental quantities.

Computational cost: The computational cost of one Newton-Raphson iteration is approximately the same for the Q1/P0, Q1-F-bar and the proposed elements for finite element models that consist of the same number of 
nodes. Therefore, the numerical scheme that requires fewer iterations per load step and fewer load steps for completing the simulation is considered to be computationally efficient.

\subsection{Verification with analytical solution}

The accuracy of the proposed finite element formulation is at first verified by studying an example with an analytical solution that has been previously studied in Hennan et al. [32], Liu et al. [114], Sharma and Joglekar $[73,74]$. We consider a plane-strain model (2D) and a 3D model with the original side length $L \mathrm{~mm}$, as shown in Fig. 2. The material model is the truly incompressible Gent one with a shear modulus $\mu=1 \mathrm{~Pa}$ while the relative permittivity is taken as $\varepsilon_{r}=1 / 8.854$.

For the plane-strain model, the edges $\mathrm{AB}$ and $\mathrm{AD}$ are constrained from moving in the normal direction, and the edge $\mathrm{AB}$ is held at a zero voltage while a positive voltage is applied on the edge $\mathrm{CD}$. For the 3D model, faces $\mathrm{ADHE}, \mathrm{ABFE}$, and $\mathrm{ADCB}$ are constrained from moving in the normal directions and a positive voltage is applied on the face EHGF while the prescribed electric potential on the face ADCB is zero. Simulations are performed for five different values of $I_{m}$ using the finite element meshes shown in Fig. 3.

The values of principal stretch $(\lambda)$ obtained from the simulations are plotted in Figs. $4 \mathrm{a}$ and $4 \mathrm{~b}$, respectively, for the plane-strain and 3D models, against the normalised potential $\left(\bar{\phi}=\frac{\phi}{L} \sqrt{\frac{\varepsilon}{\mu}}\right)$. As shown, the results obtained from the proposed scheme are in excellent agreement with the analytical solution for both the planestrain and 3D models. The contour plots of principal stretch $(\lambda)$ presented in Fig. 3 show a constant value throughout the domain.

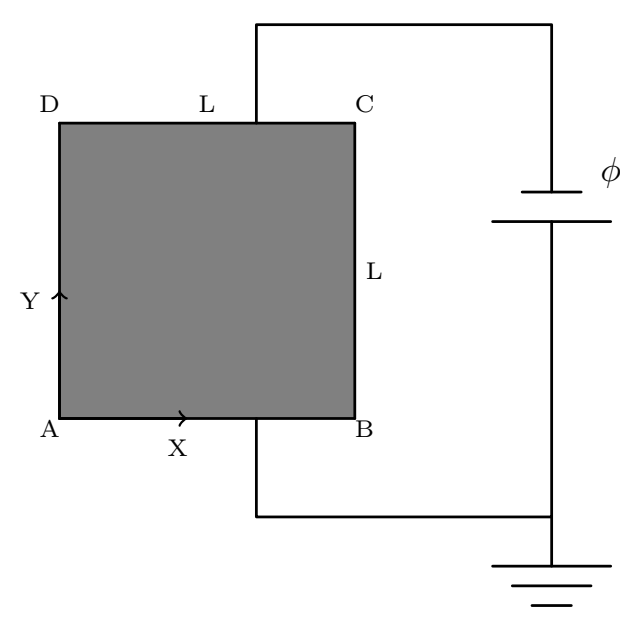

(a) Plane-strain model

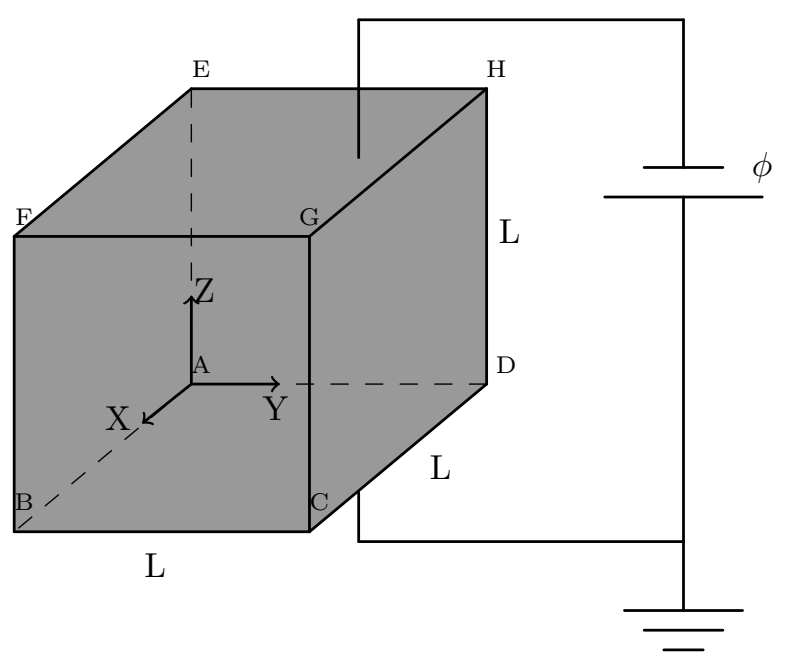

(b) 3D model

Figure 2: Analytical solution example: problem setup for (a) plane-strain model and (b) 3D model.

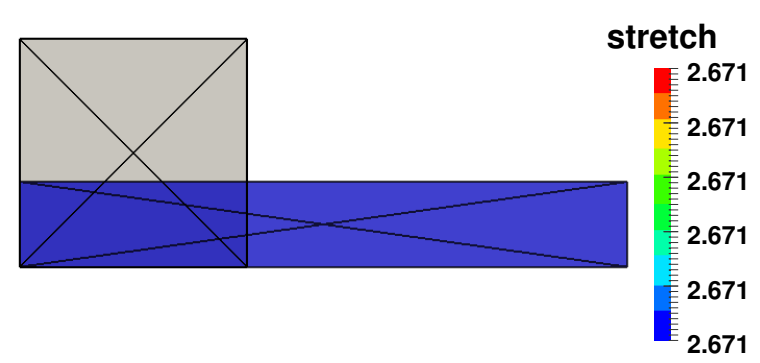

(a) Plane-strain model

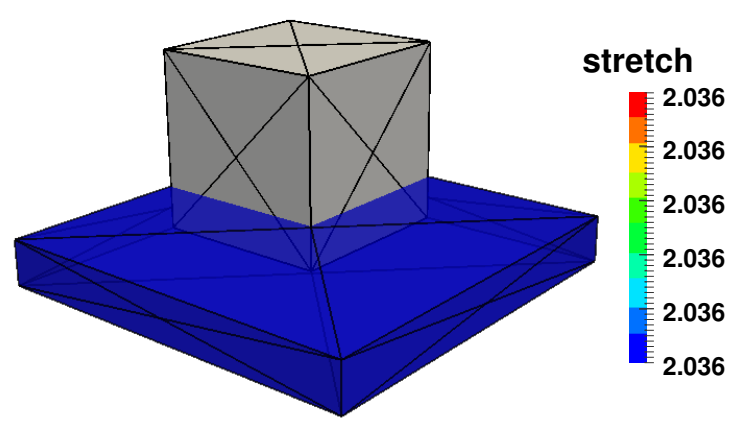

(b) 3D model

Figure 3: Analytical solution example: original and deformed meshes along with the nodal contours of the principal stretch for $I_{m}=7$ for the BT2/BT1/BT2 element. 


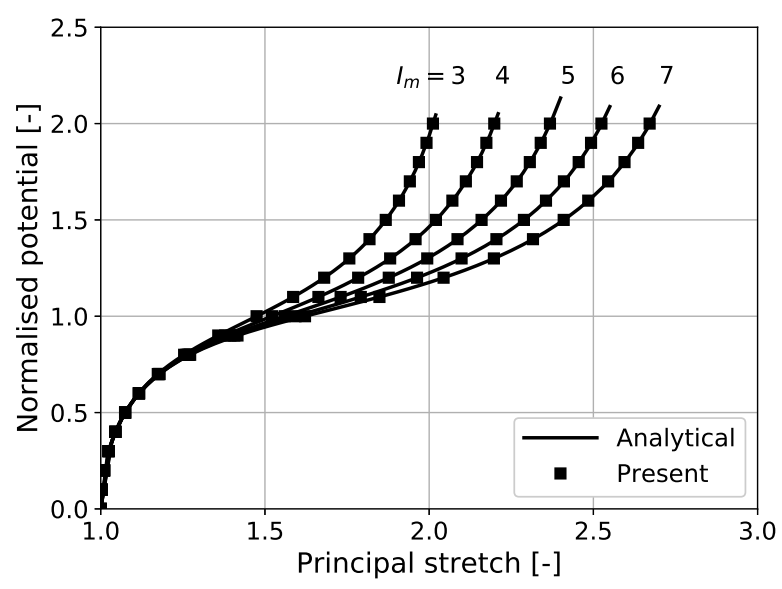

(a) Plane-strain model

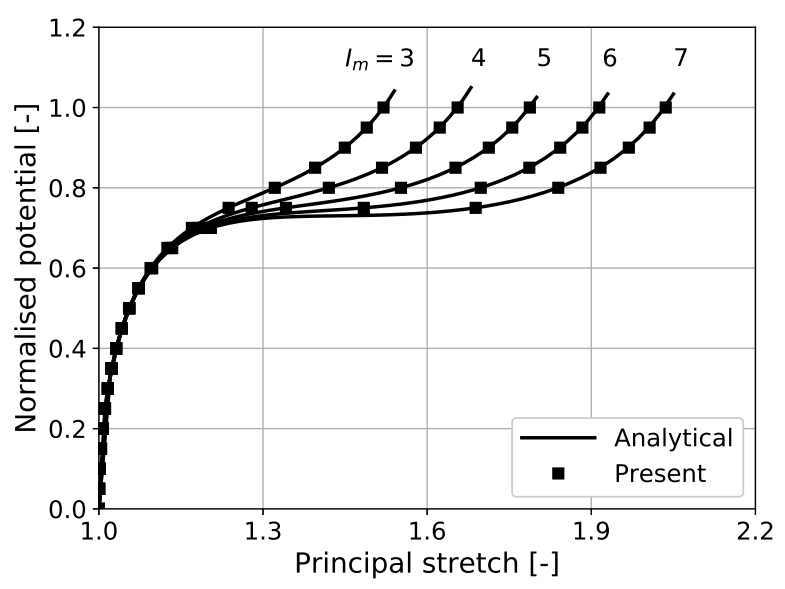

(b) 3D model

Figure 4: Analytical solution example: comparison of numerical values of principal stretch obtained with the BT2/BT1/BT2 element against the analytical solution for (a) plane-strain model and (b) 3D model. The results are indistinguishable for the other higher-order elements.

\subsection{Thin bi-layer bending actuator in plane-strain}

In this example, we demonstrate the suitability and effectiveness of the proposed finite element framework for electro-active polymeric actuators undergoing a bending deformation. For this purpose, we consider a thin bi-layer actuator whose geometry and boundary conditions are as shown in Fig. 5a where the length of the beam is $L=20 \mathrm{~mm}$ and the thickness of each layer is $t_{0}=0.5 \mathrm{~mm}$. The interface between the two layers is grounded and a positive voltage of $\phi=3 \mathrm{kV}$ is applied on the top edge. The material model is taken as the Neo-Hookean model with a shear modulus, $\mu=10^{4} \mathrm{~Pa}$ and a relative permittivity, $\varepsilon_{r}=5$. Two different values of the bulk modulus, $10^{7} \mathrm{~Pa}$ and $10^{9} \mathrm{~Pa}$ are considered in order to demonstrate the computational advantages of the proposed framework.

Simulations are performed on five successively refined meshes starting with the discretisations shown in Fig. 5. Results obtained with the different element types are assessed by studying the variation of vertical displacement of the top right corner of the beam, point A in Fig. 5a, with respect to mesh refinement. As expected, the difference in the displacement response obtained with two different values of the bulk modulus for all the element types considered in this work is negligible.

The variation of the vertical displacement of point A obtained with different element types for $\kappa=10^{9}$ $\mathrm{Pa}$, as presented in Fig. 6 illustrates that results obtained with the proposed scheme converge as the mesh is refined and that the converged value matches well with the results obtained with the other formulations. From the graph, it can also be observed that accurate results can be obtained with the proposed element as well as with the other higher-order elements using coarse meshes: the result obtained with the coarsest mesh using the BT2/BT1/BT2 element is on par with that obtained with the twice refined mesh with the Q1/P0 and Q1-F-bar elements, and the result obtained with the second mesh using the BT2/BT1/BT2 element is already a converged value. Another noteworthy point from this graph is that the Q2/P1/Q2 element is stiffer when compared with the Q2/Q1/Q2 element. Contour plots of the nodal pressure presented in Fig. 7 illustrate that the pressure field obtained with Q1/P0 and BT2/BT1/BT2 elements is indistinguishable from each other.

While the numerical results computed with all the element types converge to the same limit as the mesh is refined, the distinction in different formulations lies in the amount of computing time required for the successful completion of each simulation. As tabulated in Table. 3, all the mixed elements, Q1/P0, P2/P1/P2, Q2/P1/Q2, $\mathrm{Q} 2 / \mathrm{Q} 1 / \mathrm{Q} 2$ and BT2/BT1/BT2, require only 10 load steps for both values of the bulk modulus while the number of load steps required with Q1-F-bar and BT2-F-bar elements is not only significantly higher but also increases with the increase in the value of the bulk modulus. For example, for $\kappa=10^{9} \mathrm{~Pa}, \mathrm{Q} 1-\boldsymbol{F}$-bar and BT2-F-bar elements require 100 load steps which is 10 times more than that required with the mixed elements. Thus, the proposed framework results in significant computational benefits due to its ability to compute accurate numerical results using coarse meshes and fewer load steps. 


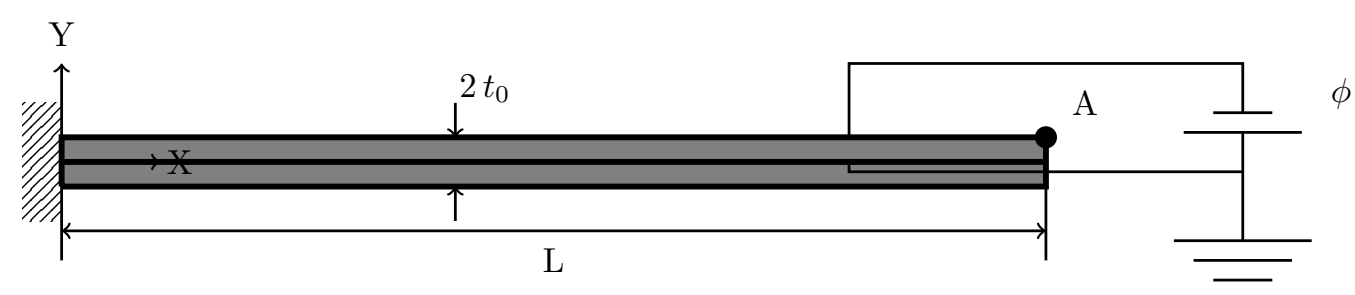

(a)

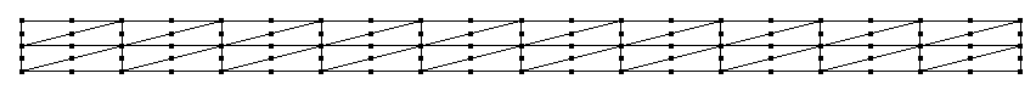

(b)

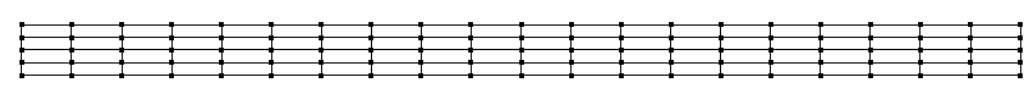

(c)

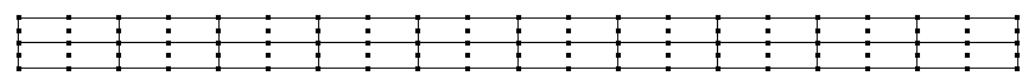

(d)

Figure 5: Bending actuator in plane strain: (a) geometry and boundary conditions (b) $(10 \times 2) \times 2$ triangular mesh for the BT2-F-bar, BT2/BT1/BT2 and P2/P1/P2 elements, (c) $20 \times 4$ quadrilateral mesh for the Q1/P0 and Q1-F-bar elements, and (d) $10 \times 2$ quadrilateral mesh for the Q2/P1/Q2 and Q2/Q1/Q2 elements.

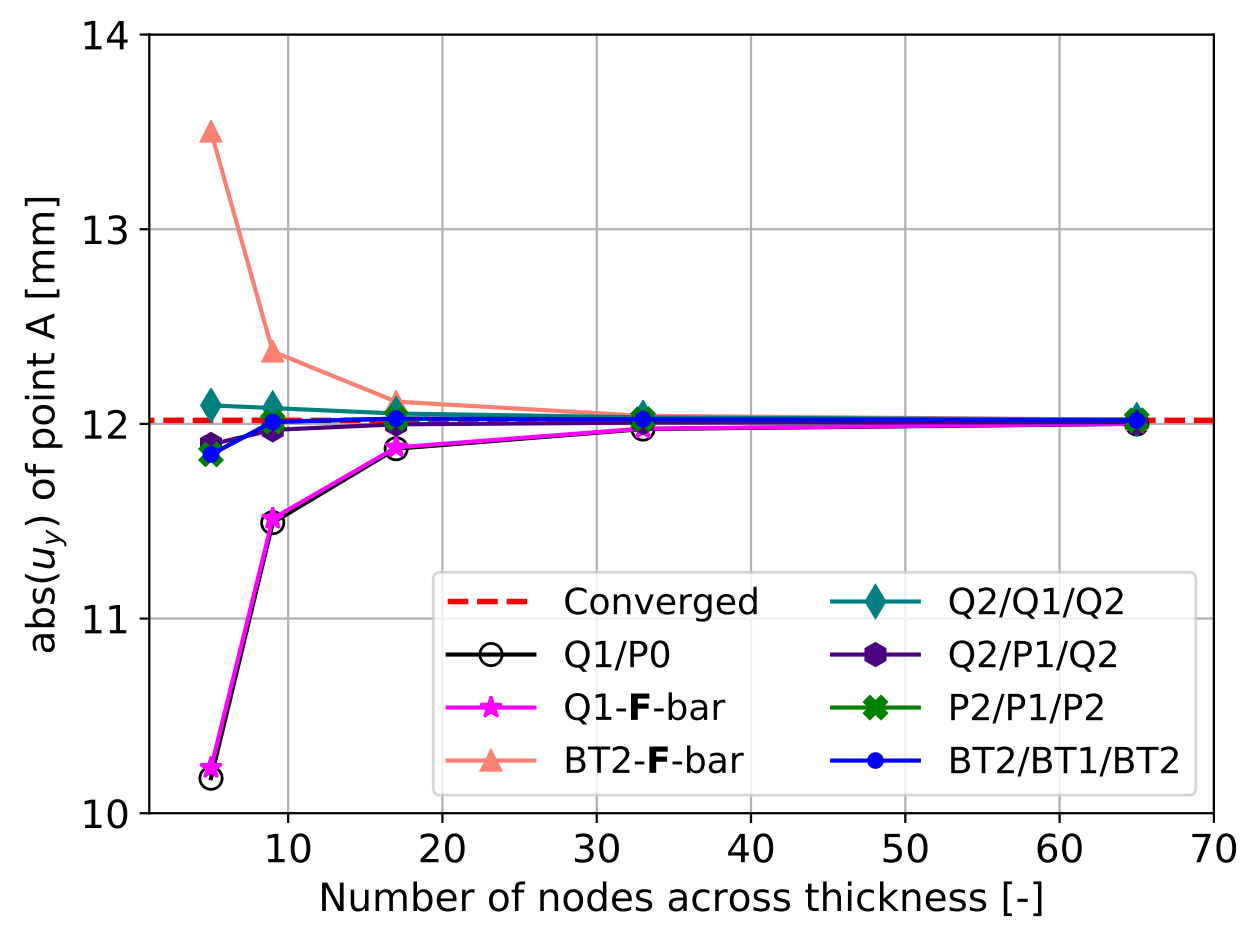

Figure 6: Bending actuator in plane strain: variation of the absolute value of the vertical displacement of point A against the number of nodes across the thickness using different element types for the case with $\kappa=10^{9} \mathrm{~Pa}$. 


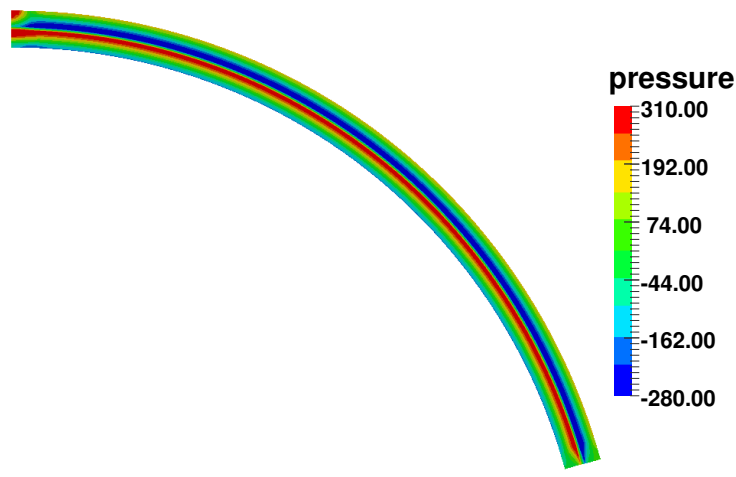

(a)

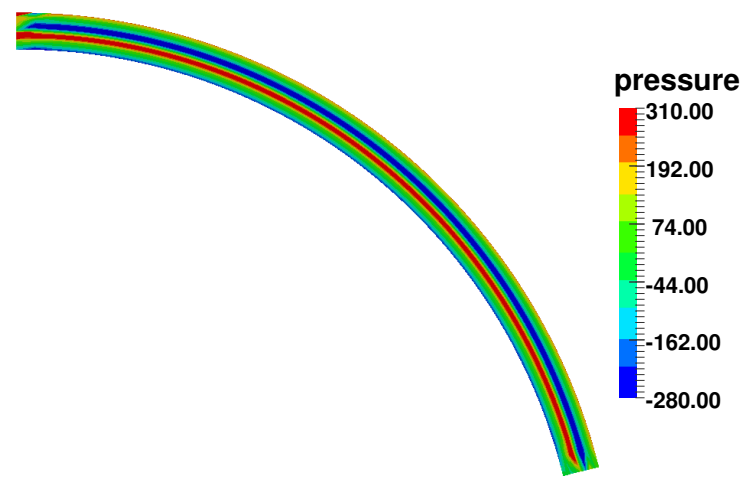

(b)

Figure 7: Bending actuator in plane strain: nodal contours of pressure field obtained for $\kappa=10^{9} \mathrm{~Pa}$ with (a) $80 \times 16$ mesh of Q1/P0 element and (b) $(40 \times 8) \times 2$ mesh with BT2/BT1/BT2 element.

\begin{tabular}{|c|c|c|c|c|}
\hline \multirow{2}{*}{$\begin{array}{c}\text { Number of nodes } \\
\text { across thickness }\end{array}$} & \multicolumn{2}{|l|}{ Mixed elements } & \multicolumn{2}{c|}{ Q1-F-bar \& BT2-F-bar elements } \\
\cline { 2 - 5 } & $\kappa=10^{7} \mathrm{~Pa}$ & $\kappa=10^{9} \mathrm{~Pa}$ & $\kappa=10^{7} \mathrm{~Pa}$ & $\kappa=10^{9} \mathrm{~Pa}$ \\
\hline 5 & 10 & 10 & 50 & 100 \\
17 & 10 & 10 & 50 & 100 \\
33 & 10 & 10 & 50 & 100 \\
65 & 10 & 10 & 50 & 100 \\
\hline
\end{tabular}

Table 3: Bending actuator in plane strain: number of uniform load steps required for the successful completion of each simulation with ten or less number of Newton-Raphson iterations for each load step. Mixed elements refer to the Q1/P0, Q2/Q1/Q2, Q2/P1/Q2, P2/P1/P2 and BT2/BT1/BT2 elements.

\subsection{Thin bi-layer bending actuator in $3 D$}

This example is a three-dimensional extension of the bi-layer cantilever beam studied in the previous Section 5.2. The dimensions of the beam are: length $=20 \mathrm{~mm}$, thickness of each layer $=0.5 \mathrm{~mm}$, and width $=$ $4 \mathrm{~mm}$. The material is assumed to be Neo-Hookean with a shear modulus, $\mu=10^{4} \mathrm{~Pa}$ and a bulk modulus, $\kappa=10^{9} \mathrm{~Pa}$. The relative permittivity $\left(\varepsilon_{r}\right)$ is taken as 5 . The interface layer is held at a zero potential and a positive voltage of $3.2 \mathrm{kV}$ is applied on the top surface, causing the beam to bend downwards.

Numerical simulations are carried out on successively refined four finite element meshes starting with the discretisations shown in Fig. 8. The variation of Y-displacement of point A presented in Fig. 9 illustrates that convergence behaviour is consistent with that observed in the plane-strain example. From the graph, it is also clear that the third mesh using the proposed element already gives a converged solution while Q1/P0 and Q1-F-bar elements require further mesh refinement in order to achieve the converged value. Moreover, while the results obtained with Q1/P0 and Q1-F-bar elements are almost identical, Q1-F-bar element requires 1000 load steps while Q1/P0, BT2/BT1/BT2 and other mixed elements require only 10 load steps. We can also notice from the graph that the Q2/P1/Q2 element is stiffer than the Q2/Q1/Q2 and BT2/BT1/BT2 elements. The difference in the displacement obtained with the Q2/P1/Q2 and Q2/Q1/Q2 elements is more pronounced when compared with the results from the plane-strain problem. Although the Q2/P1 element is inf-sup stable, it still yields inferior quality results when compared with the other inf-sup stable elements because of the poor quality of the approximation for the pressure field within a Q2/P1 element. Such behaviour of the Q2/P1 element is characteristic of incompatible approximation space used for the pressure field.

Additionally, the contour plots of element-wise pressure presented in Fig. 10 indicate spurious modes in the pressure field obtained with the Q1/P0 element whereas the same field obtained with the proposed element is 
free from oscillations. Therefore, accurate numerical results can be obtained with the proposed element using fewer computational resources when compared with the widely-used Q1/P0 and Q1-F-bar elements for computational electromechanics, without even considering the amount of resources wasted in the crashed simulations with the Q1-F-bar elements when insufficient number of load steps are chosen. This computationally-appealing behaviour of the proposed element is because of the use of second-order basis functions for the displacement field.

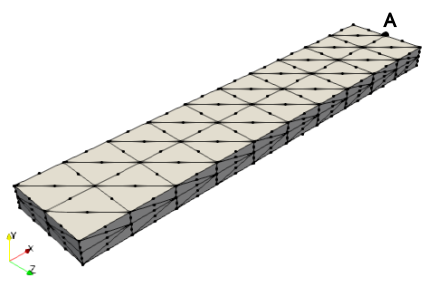

(a)

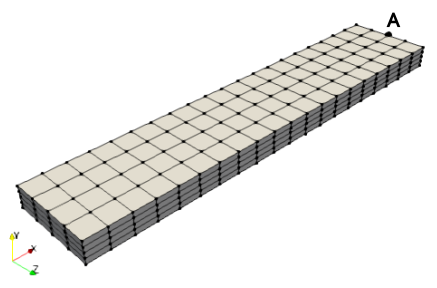

(b)

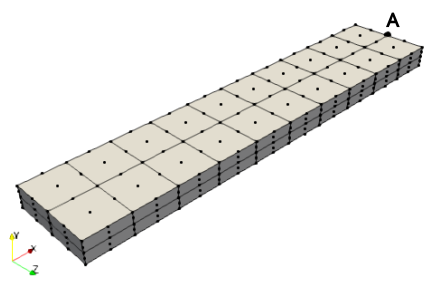

(c)

Figure 8: Bending actuator in 3D: (a) $(10 \times 2 \times 2) \times 6$ tetrahedral mesh for the BT2/BT1/BT2 and P2/P1/P2 elements, (b) $20 \times 4 \times 4$ hexahedral mesh for the Q1/P0 and Q1-F-bar elements and (c) $10 \times 2 \times 2$ hexahedral mesh for the $\mathrm{Q} 2 / \mathrm{P} 1 / \mathrm{Q} 2$ and $\mathrm{Q} 2 / \mathrm{Q} 1 / \mathrm{Q} 2$ elements.

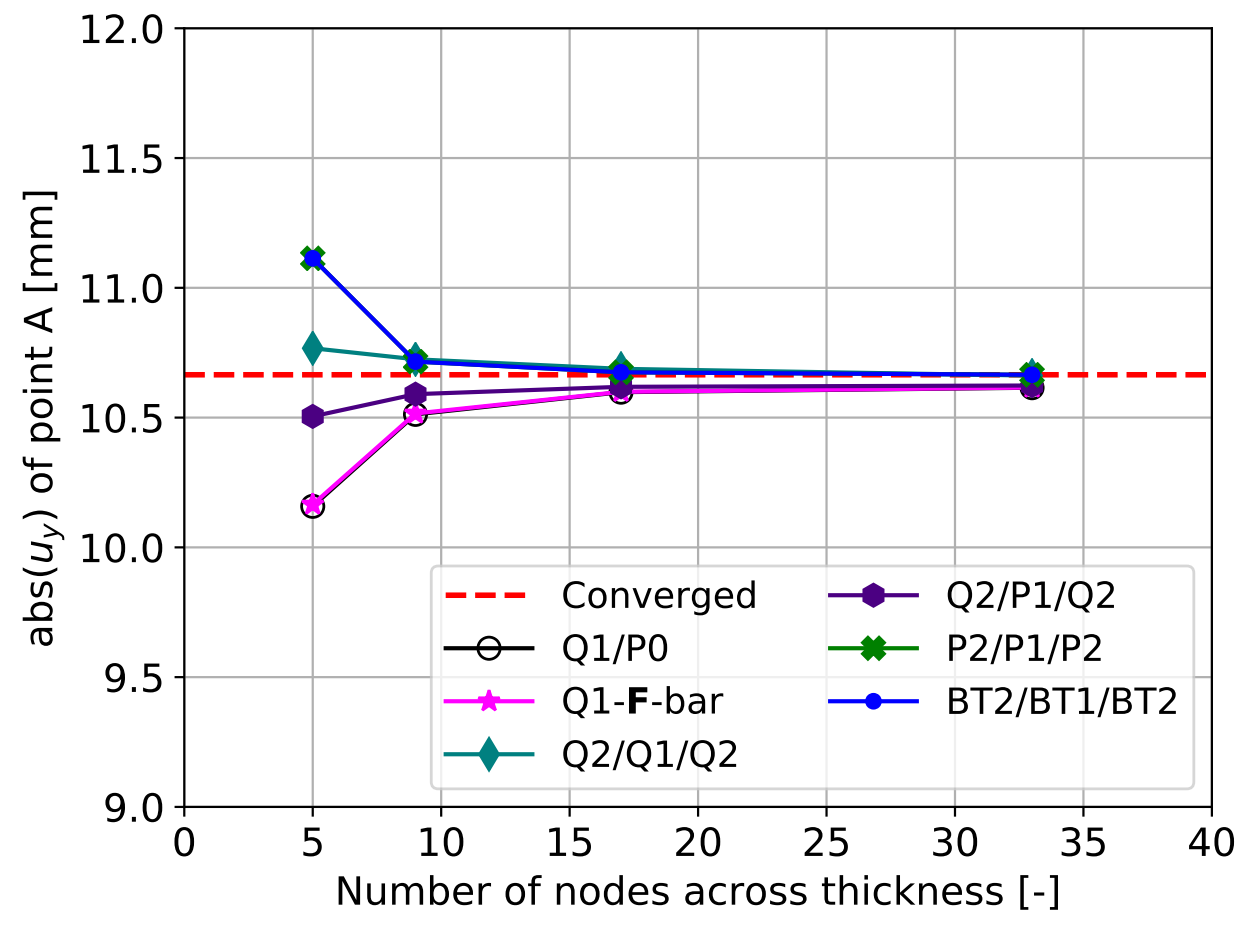

Figure 9: Bending actuator in 3D: variation of the absolute value of the vertical displacement of point A against the number of nodes across the thickness using different formulations. 


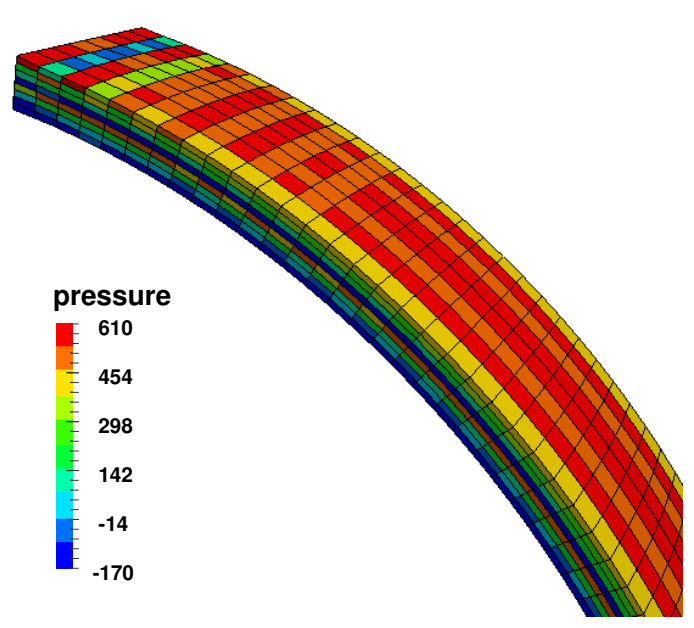

(a) $\mathrm{Q} 1 / \mathrm{P} 0$

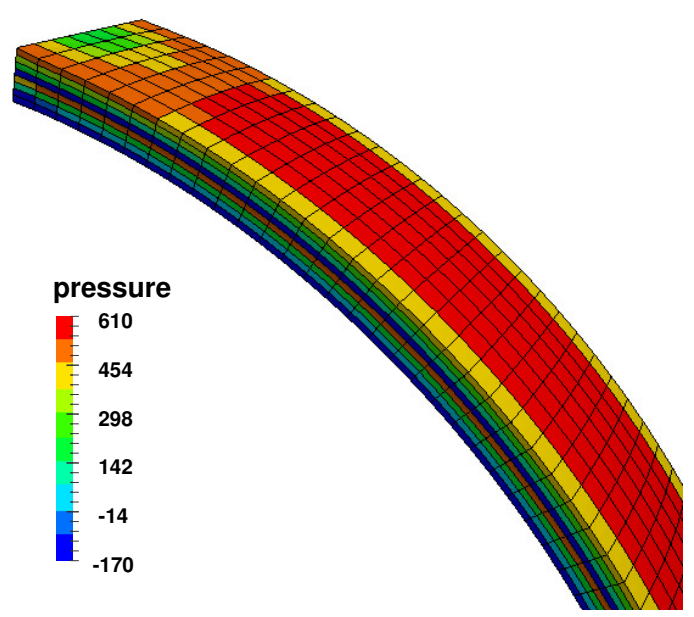

(c) $\mathrm{Q} 2 / \mathrm{P} 1 / \mathrm{Q} 2$

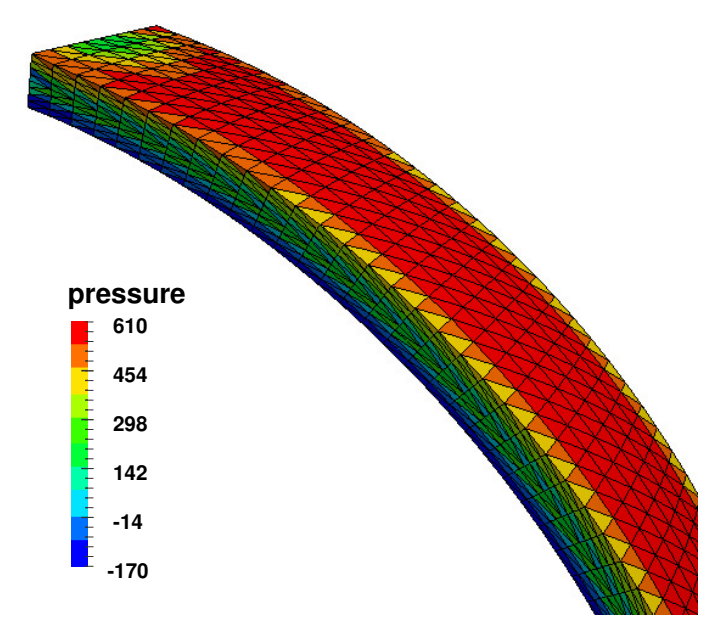

(b) BT2/BT1/BT2

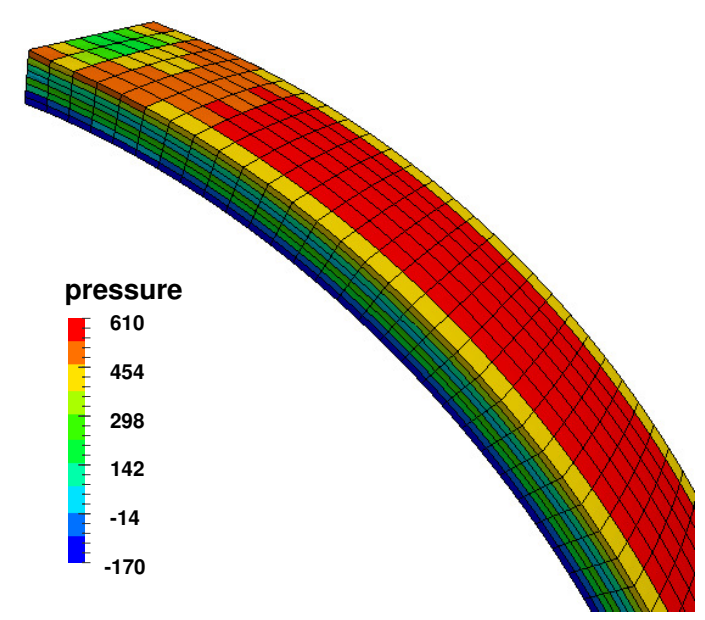

(d) Q2/Q1/Q2

Figure 10: Bending actuator in 3D: contour plot of element-wise pressure obtained with different element types.

\subsection{Spherical gripper}

In this example, we demonstrate the suitability of the proposed finite element framework to accurately capture large deformation behaviour in real-world applications of electromechanical actuators. For this, we consider the example of a thin bi-layer spherical gripper previously studied in Ask et al. [2] and Bishara and Jabareen [7]. The gripper is made of 12 arms, as shown in Fig. 11a, with each arm made of two electro-active layers. The radius of the sphere at the interface layer is $20 \mathrm{~mm}$, and the thickness of each layer is $0.5 \mathrm{~mm}$. The opening angle at the top and bottom holes is $30^{\circ}$. The mechanical boundary and loading conditions are such that the topmost face of the gripper is fixed and the rest of the faces are traction free. For the electrical loading, the interface layer is held at a zero voltage, and a positive voltage of $6 \mathrm{kV}$ is applied on the inner side of the arm. Due to the symmetry of boundary and loading conditions, only one half of the arm, as shown in Fig. 11b, is considered for the analysis. The finite element meshes used for this problem are shown in Figs. 11c, 11d and $11 \mathrm{e}$; all the meshes consist of 4545 nodes.

The constitutive law is assumed to the Gent model with $\mu=2.6 \times 10^{4} \mathrm{~Pa}$ and $I_{m}=7$, and the relative permittivity is $\varepsilon_{r}=4.5$. To demonstrate the advantages of the proposed framework over Q1/P0 and Q1-F-bar elements, we consider two values of the bulk modulus, $\kappa=2.6 \times 10^{7} \mathrm{~Pa}$ and $2.6 \times 10^{9} \mathrm{~Pa}$. The variation of displacement of point A obtained with $\kappa=2.6 \times 10^{7} \mathrm{~Pa}$ with the BT2/BT1/BT2 element matches well with the values obtained with the Q1/P0, Q1-F-bar and Q2/Q1/Q2 elements, as presented in Fig. 12. The relatively stiff behaviour of the Q2/P1/Q2 element when compared with the other elements, which is consistent with the response in the previous example, is also evident from the graph. Deformed shapes at two different load steps are presented in Fig. 13 to illustrate the superior quality of numerical results obtained with the BT2/BT1/BT2 
element when compared with the $\mathrm{Q} 2 / \mathrm{P} 1 / \mathrm{Q} 2$ element. The deformed shapes of the gripper, post-processed for all the arms, are shown in Fig. 14 under different applied voltages.

Although the displacement response obtained with different schemes is in good agreement with each other, the main differences are in terms of the computational cost. For $\kappa=2.6 \times 10^{7} \mathrm{~Pa}$, all the mixed elements, $\mathrm{Q} 1 / \mathrm{P} 0, \mathrm{Q} 2 / \mathrm{P} 1 / \mathrm{Q} 2$ and Q2/Q1/Q2 and BT2/BT1/BT2, require only 50 load steps while the Q1-F-bar element requires 250 load steps. This behaviour of the Q1-F-bar element in requiring a significantly higher number of load steps is consistent with the observations made in the previous examples. Due to its substantial computational cost, the Q1-F-bar element is discarded henceforth in this paper.

The convergence of Newton-Raphson iterations for the last 10 load steps for $\kappa=2.6 \times 10^{7}$ Pa shown in Fig. 15 illustrates that the proposed scheme achieves residual values that are two to three orders of magnitude lower than those of the Q1/P0 element for the same number of iterations at each load step. It is also observed that increasing the value of the bulk modulus to $2.6 \times 10^{9} \mathrm{~Pa}$ does not affect the convergence of the proposed scheme while the convergence of iterations not only deteriorates but also stalls at the 48th load step for the $\mathrm{Q} 1 / \mathrm{P} 0$ element, as shown in Fig. 16. For the tolerance of $10^{-4}$, the simulation with Q1/P0 element crashes at the 48th load step. Hence, in order to achieve convergence, the tolerance has been lowered to $10^{-3}$, as shown in Fig. 17. It is also evident from Fig. 17 that, for the same number of iterations, the proposed scheme achieves residual values that are four orders of magnitude lower. We highlight at this point that this unappealing convergence behaviour, which is symptomatic of the elimination of pressure DOFs from the global system, is also common to the Q2/P1/Q2 element.

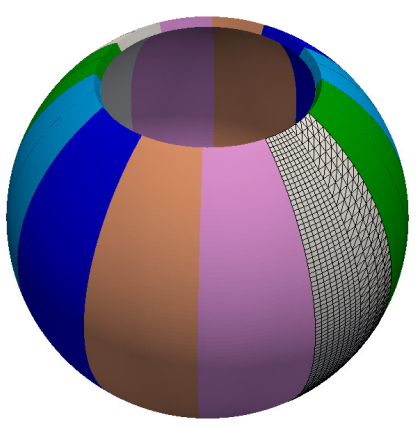

(a)

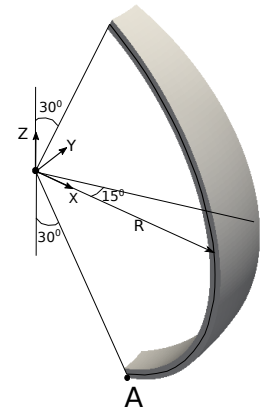

(b)

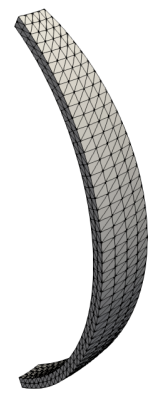

(c)

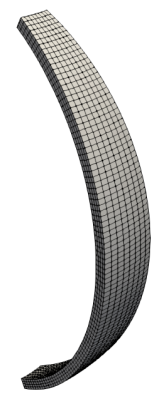

(d)

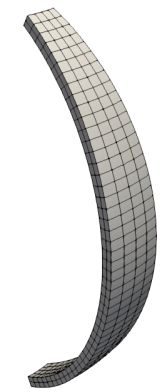

(e)

Figure 11: Spherical gripper: (a) the gripper with 12 arms, (b) geometry description for a half portion of a single arm, (c) $(50 \times 4 \times 2) \times 6$ mesh for the BT2/BT1/BT2 element, (d) $100 \times 8 \times 4$ mesh for the Q1/P0 element and (e) $50 \times 4 \times 2$ mesh for the Q2/P1/Q2 and Q2/Q1/Q2 elements. 


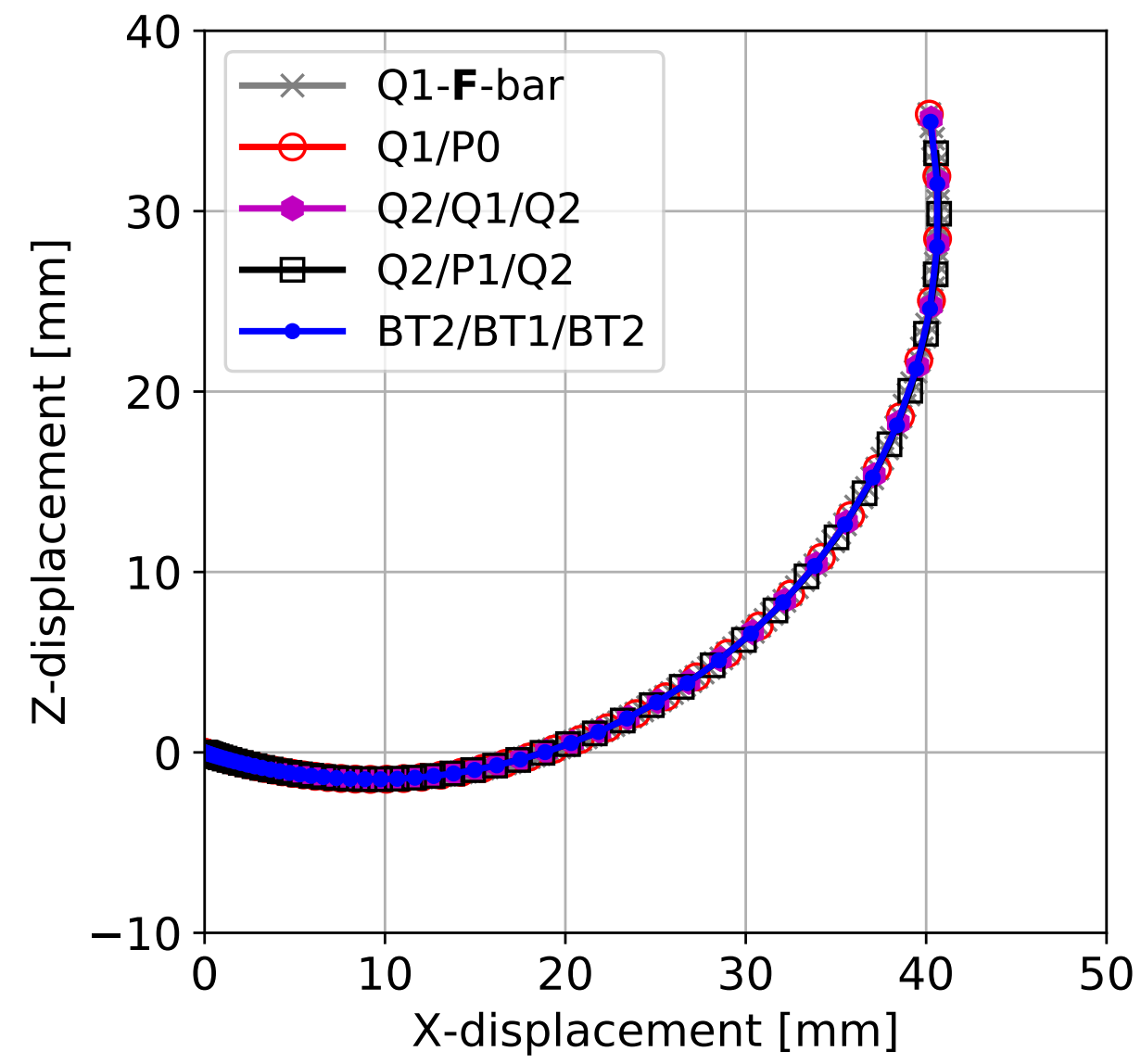

Figure 12: Spherical gripper: variation of the displacement of point A obtained with different element types for $\kappa=$ $2.6 \times 10^{7} \mathrm{~Pa}$.

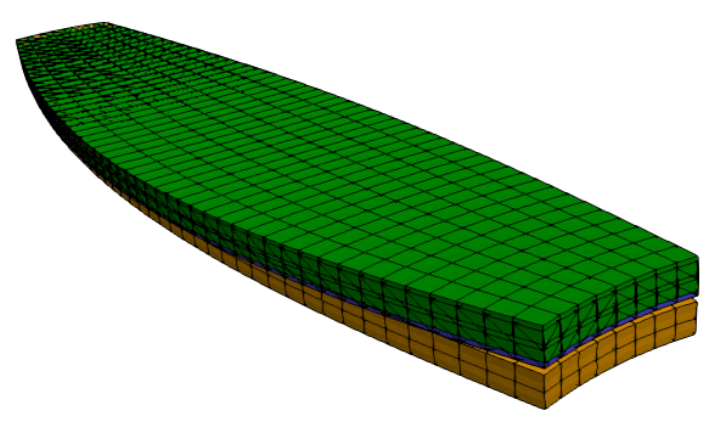

(a) 45th load step, $\phi=5.4 \mathrm{kV}$

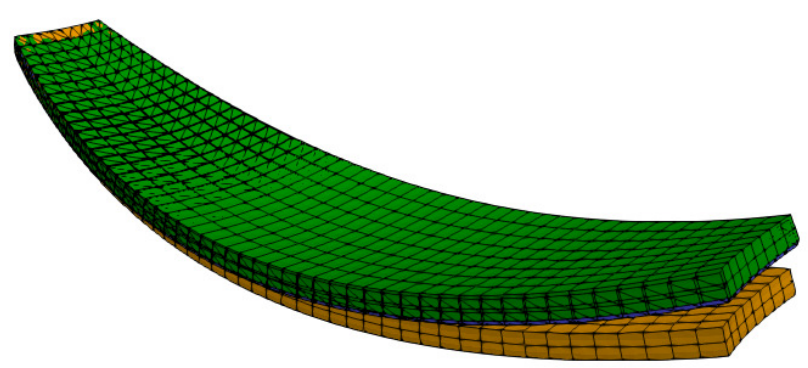

(b) 50 th load step, $\phi=6.0 \mathrm{kV}$

Figure 13: Spherical gripper: deformed shapes of the gripper at two different values of applied voltage. Green: Q2/Q1/Q2 element, Blue: BT2/BT1/BT2 element, and Orange: Q2/P1/Q2 element. Note that there is no significant difference between the deformed shapes obtained with Q2/Q1/Q2 (Green) and BT2/BT1/BT2 (Blue). 


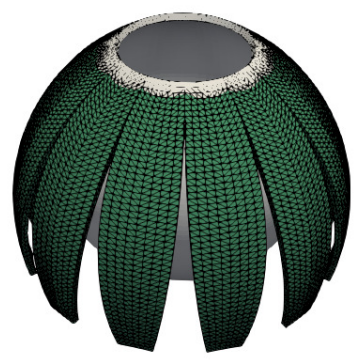

(a) $\phi=3.6 \mathrm{kV}$

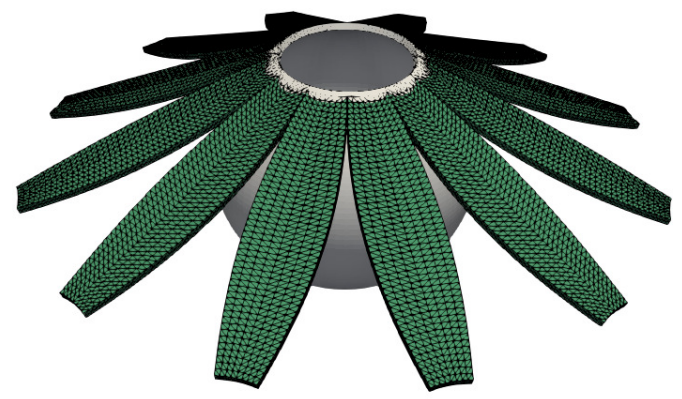

(c) $\phi=5.4 \mathrm{kV}$

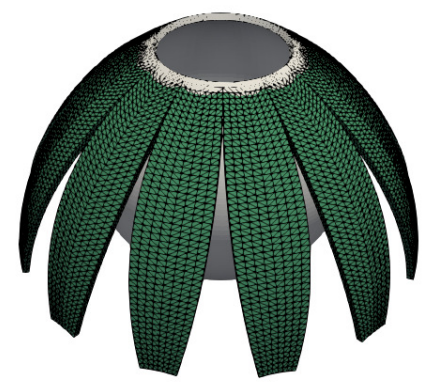

(b) $\phi=4.2 \mathrm{kV}$

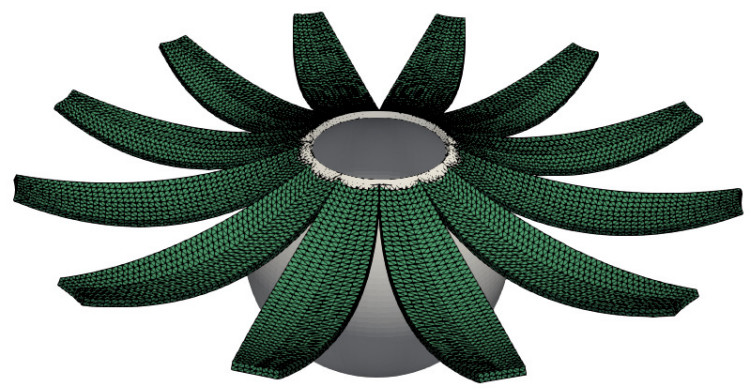

(d) $\phi=6.0 \mathrm{kV}$

Figure 14: Spherical gripper: deformed shapes of the gripper at different values of applied voltage.

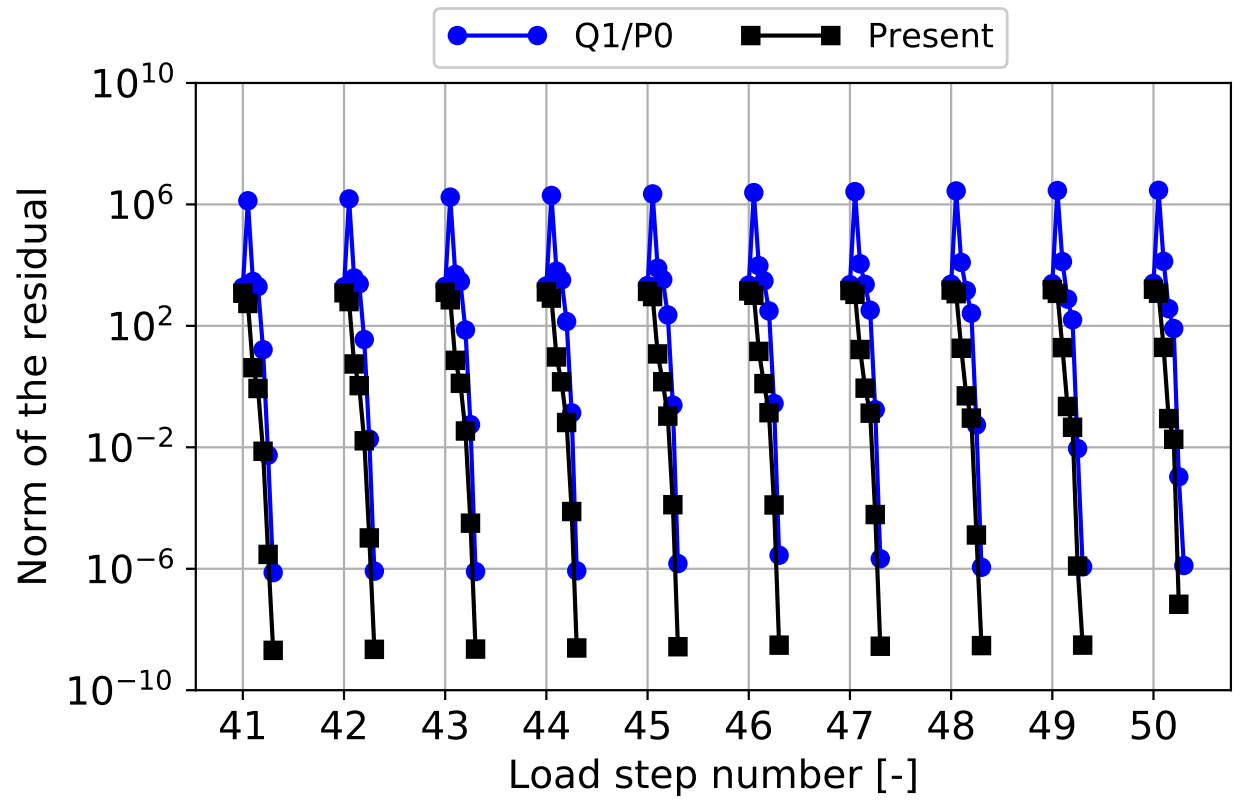

Figure 15: Spherical gripper: convergence of Newton-Raphson iterations for the last ten load steps for $\kappa=2.6 \times 10^{7} \mathrm{~Pa}$. 


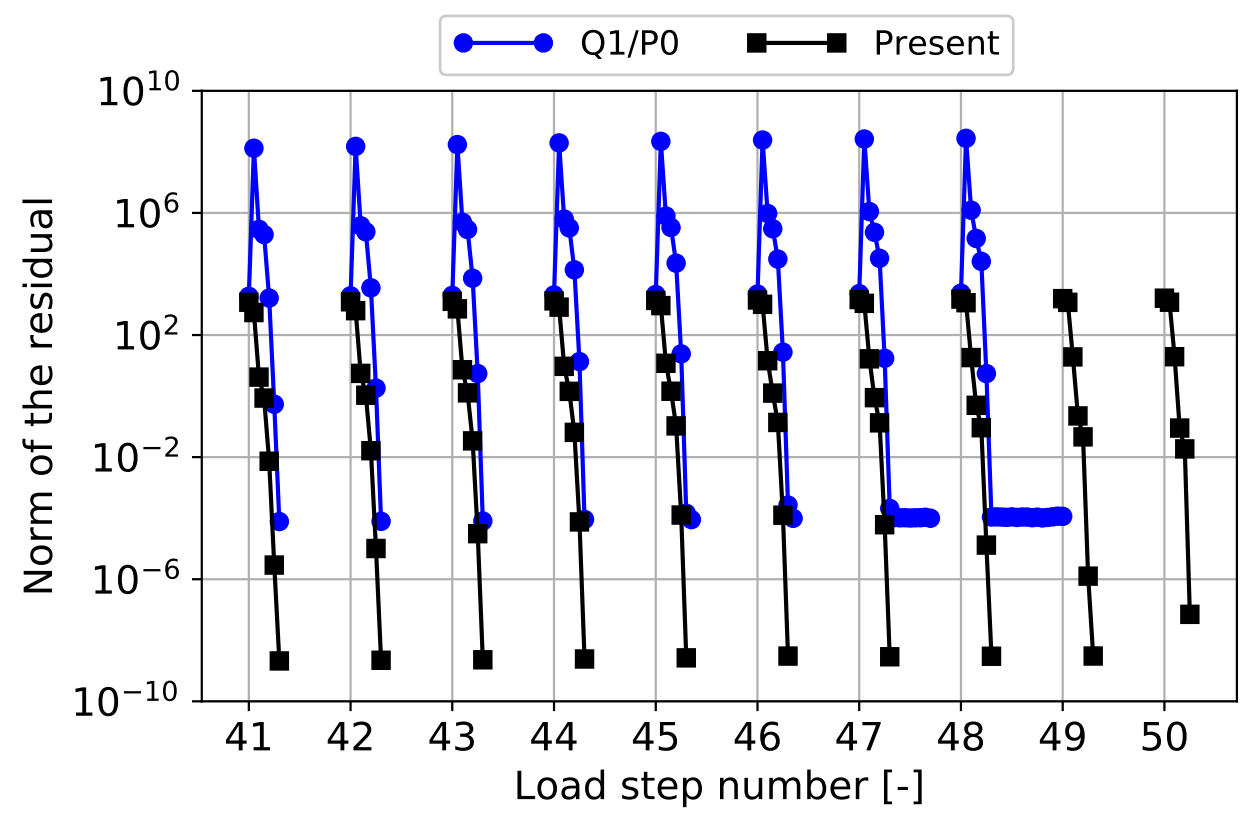

Figure 16: Spherical gripper: convergence of Newton-Raphson iterations for the last ten load steps for $\kappa=2.6 \times 10^{9} \mathrm{~Pa}$. The convergence stalls and the simulation crashes at the 48th load step for the Q1P0 element.

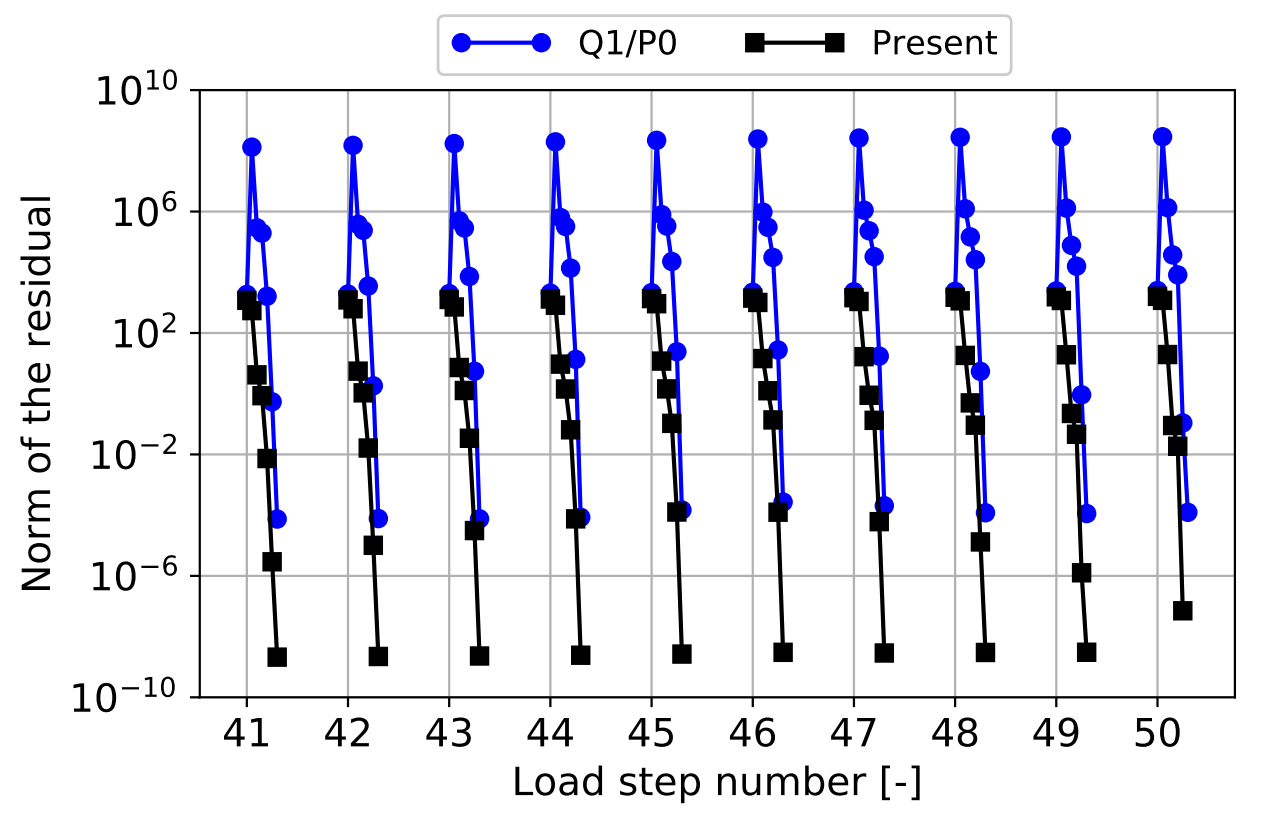

Figure 17: Spherical gripper: convergence of Newton-Raphson iterations for the last ten load steps for $\kappa=2.6 \times 10^{9} \mathrm{~Pa}$. The tolerance for the Q1/P0 element has been lowered to achieve a successful convergence.

\subsection{Very thin bi-layer bending actuator}

This example is concerned with the simulation of a very thin bi-layer bending actuator previously studied in Klinkel et al. [46]. The cantilever beam is of length $100 \mathrm{~mm}$, width $50 \mathrm{~mm}$, and the thickness of each layer $0.5 \mathrm{~mm}$. The interface layer is grounded and a positive voltage of $4.2 \mathrm{kV}$ is applied on the top face, causing the beam to bend downwards. The material model is assumed to be a truly incompressible $(\nu=0.5)$ Gent model with a shear modulus, $\mu=2.056 \times 10^{4} \mathrm{~Pa}$ and $I_{m}=5$, and the relative permittivity is taken as $\varepsilon_{r}=4.97$. 
The structured hexahedral and the unstructured tetrahedral meshes used for the simulations are shown in Fig. 18. Only one element is considered across the thickness of each layer. The hexehedral mesh consists of 8925 nodes and 850 elements while the tetrahedral mesh consists of 8635 nodes and 4932 elements. The simulations converge in 50 and 54 load steps, respectively, for the Q2/Q1/Q2 and BT2/BT1/BT2 elements. The deformed configurations of the bending actuator at different load steps obtained with the BT2/BT1/BT2 element are in good agreement with those obtained with the Q2/Q1/Q2 element, as shown in Fig. 19. This example illustrates the capability of the proposed work in successfully simulating the behaviour of very thin bending actuators using a single finite element formulation.

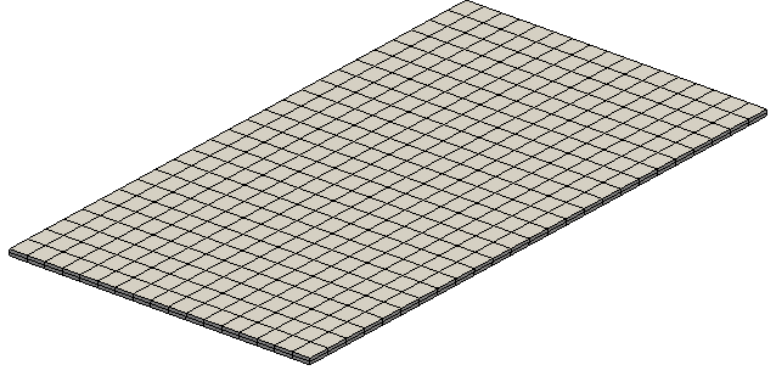

(a)

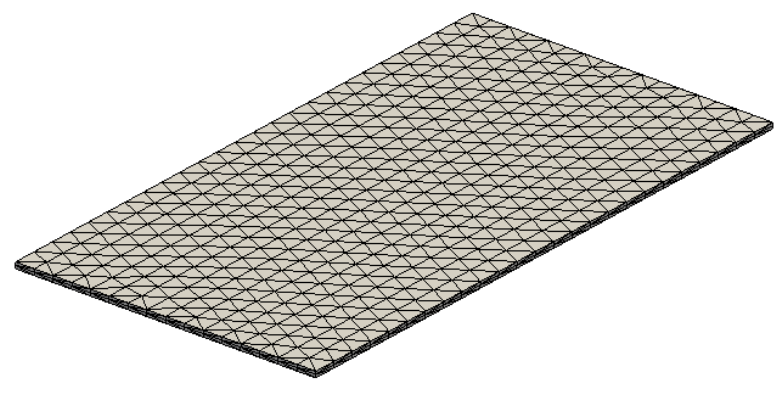

(b)

Figure 18: Thin bi-layer bending actuator: finite element meshes used with (a) Q2/Q1/Q2 element and (b) BT2/BT1/BT2 element.

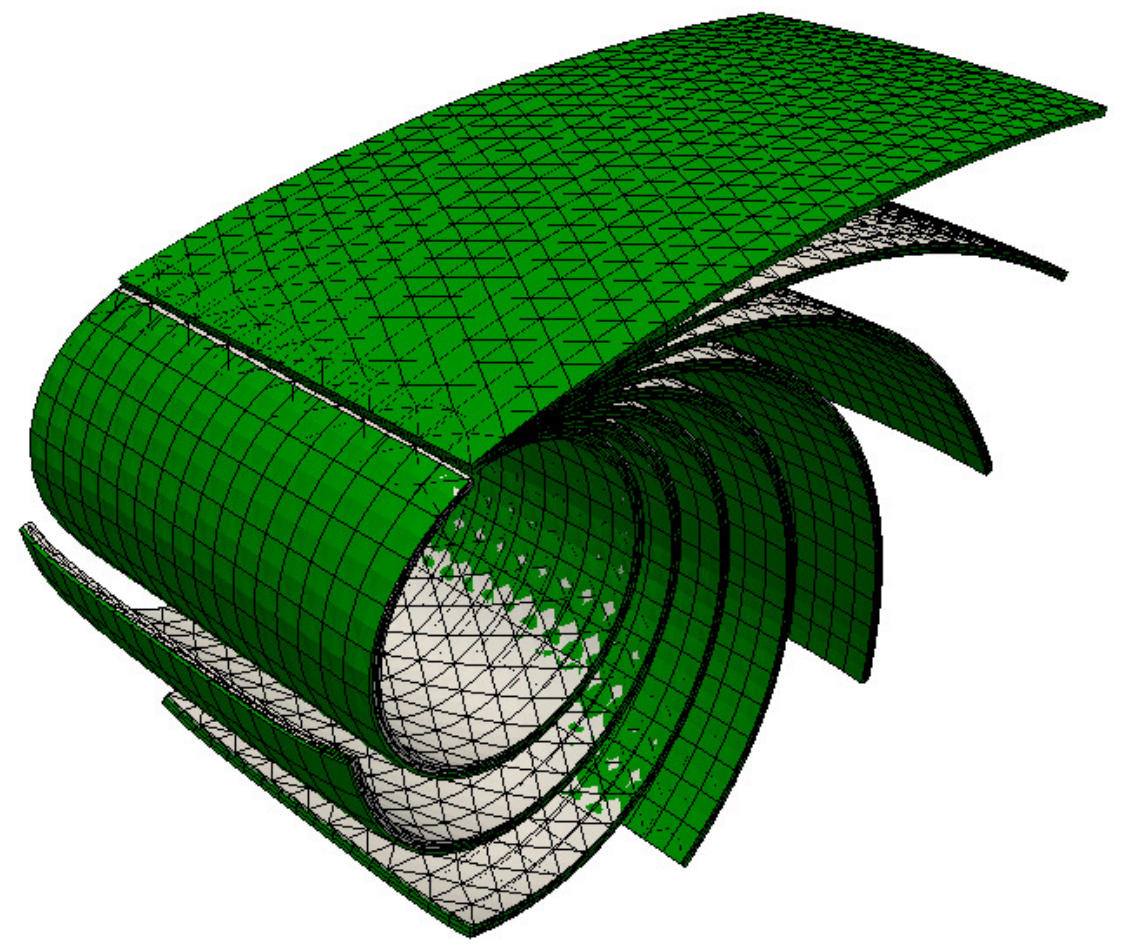

Figure 19: Thin bi-layer bending actuator: plots of deformed shapes of the actuator along with the contours of electric potential at various load steps. Green: Q2/Q1/Q2 element and White: BT2/BT1/BT2 element.

\section{Numerical examples: electro-elastodynamics}

This section is concerned with the elastodynamics simulations of practical applications of electro-active polymers. For the demonstration, first, we simulate a dielectric pump previously studied in Klassen [121]. Second, we consider a thin helical actuator excited with different frequencies of the applied voltage. Finally, 
we simulate the instabilities in thin dielectric polymers using thin semi-cylindrical and spherical shells. For all the examples in this section, the spectral radius parameter for the fully-implicit time integration scheme is taken as $\rho_{\infty}=0.5$. From among all the higher-order elements, only the BT2/BT1/BT2 element is considered hereafter for the ease of presentation of results.

\subsection{Dielectric tubular pump}

The pump is of cylindrical cross-section with a length $=50 \mathrm{~mm}$, an inner radius $=9 \mathrm{~mm}$ and a thickness $=$ $1 \mathrm{~mm}$. One pair of electrodes, each of length $10 \mathrm{~mm}$, are attached to the inner and outer cylindrical surfaces starting from one end of the cylinder, as shown in Fig. 20. The material is assumed to follow the Neo-Hookean model with a shear modulus $\mu=7.3 \times 10^{4} \mathrm{~Pa}$, a bulk modulus $\kappa=7.3 \times 10^{9} \mathrm{~Pa}$ and a density $\rho_{0}=0.0012$ $\mathrm{g} / \mathrm{mm}^{3}\left(=1200 \mathrm{~kg} / \mathrm{m}^{3}\right)$. The relative permittivity is taken as $\varepsilon_{r}=4.7$.

Simulations are performed for a fixed voltage difference of $20 \mathrm{kV}$ using two different finite element meshes for each of the BT2/BT1/BT2 and Q1/P0 elements as shown, respectively, in Figs. 21 and 22. The dynamic response of the pump is studied using the time evolution of Y-displacement of points A and B, see Fig. 20. The displacement of point A obtained with all the four meshes is plotted in Fig. 23 and the corresponding plot for point B is shown in Fig. 24.

It can be observed from Fig. 23 that there is no significant difference in the displacement response obtained with the proposed element, i.e., meshes $\mathrm{T} 1$ and $\mathrm{T} 2$, indicating a clear convergence whereas the solution obtained with the coarse hexahedral mesh (mesh H1) is noticeably different from that of the fine hexahedral mesh (mesh H2). The displacement response of point B presented in Fig. 24 shows a more pronounced difference in the solution obtained with the Q1/P0 element and the proposed BT2/BT1/BT2 element. While the solution obtained with mesh T1 matches well with that of mesh T2, the solutions obtained with the hexahedral meshes show significant differences. It is evident from Fig. 24 that the solution obtained with a coarse tetrahedral mesh (mesh T1) is significantly better than that obtained with the fine hexahedral mesh (mesh H2).

Such noticeable differences in the displacement response at a downstream point in a pumping device can have a significant impact on the accuracy of computation of flow rate delivered by the pump in multiphsyics simulations. To obtain accurate numerical solutions using the Q1/P0 element, very fine meshes need to be used, adding to a significant computational cost, and this can be avoided by using coarse meshes with the proposed finite element framework. This increased accuracy of numerical results with the proposed framework is due to the fact that second-order elements resolve mode shapes more accurately than the first-order elements. Deformed shapes of the pump along with the contour plots of element-wise pressure presented in Fig. 25 illustrate smooth pressure fields obtained with the proposed element as opposed to the pressure field with spurious modes obtained with the Q1/P0 element.

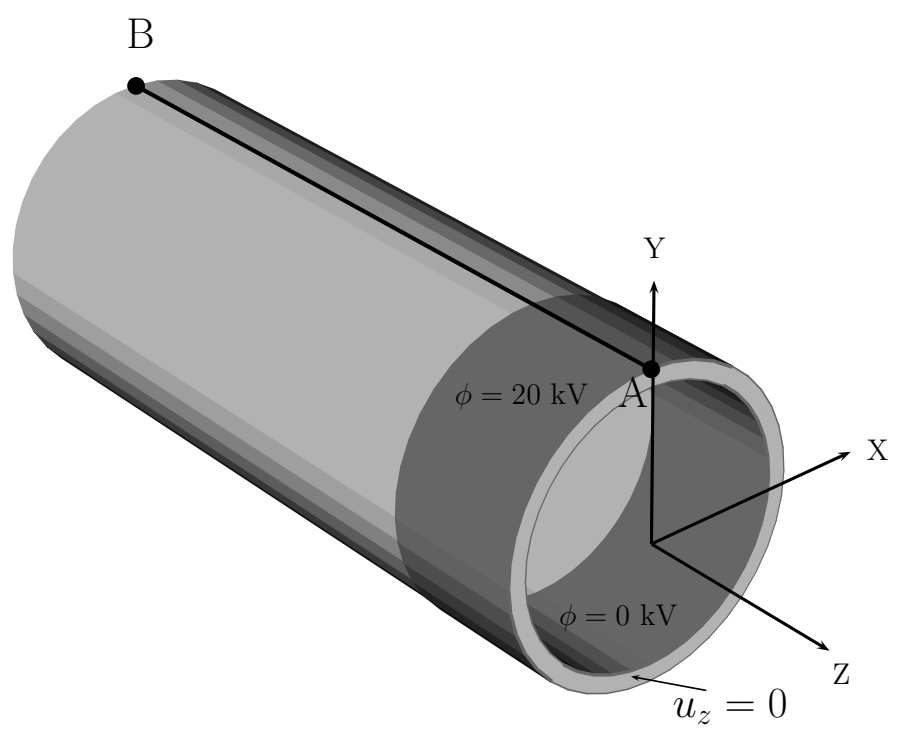

Figure 20: Dielectric pump: problem description. 


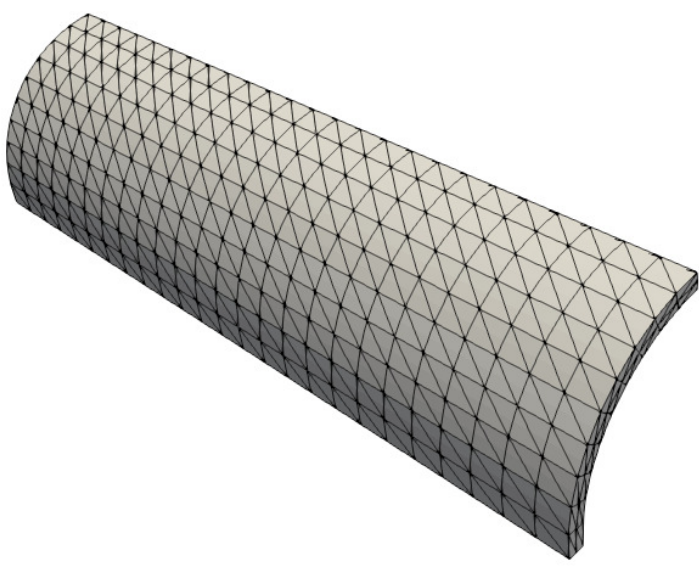

(a) Mesh T1

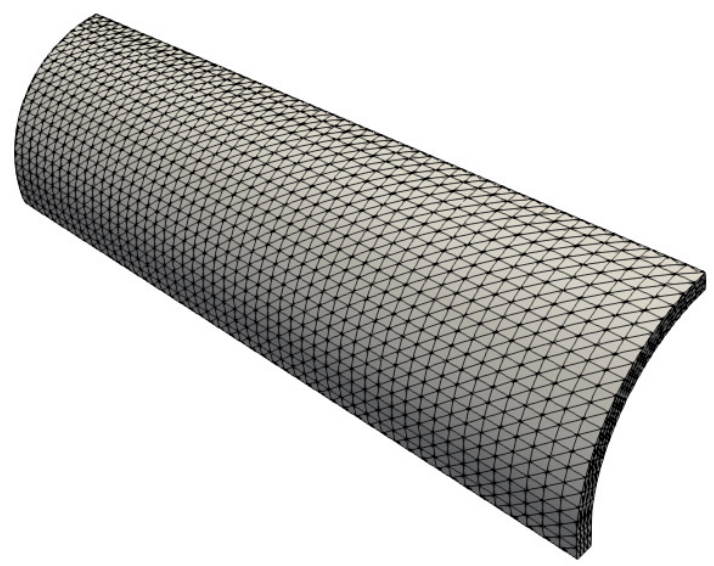

(b) Mesh T2

Figure 21: Dielectric pump: finite element meshes used for the BT2/BT1/BT2 element.

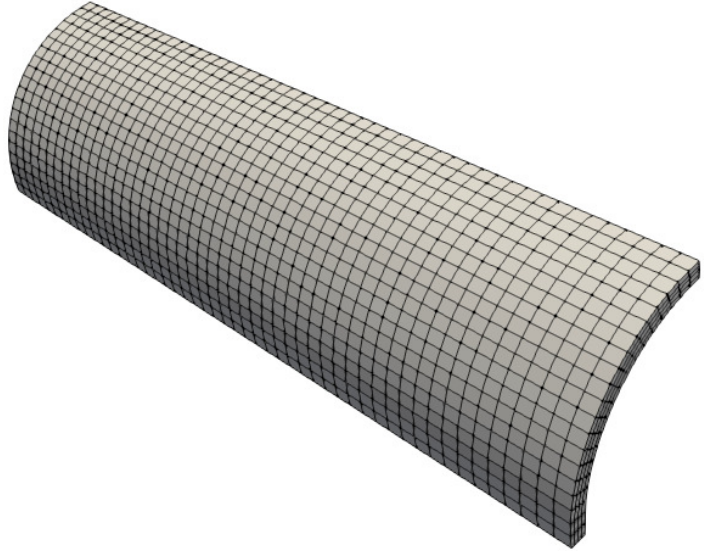

(a) Mesh H1

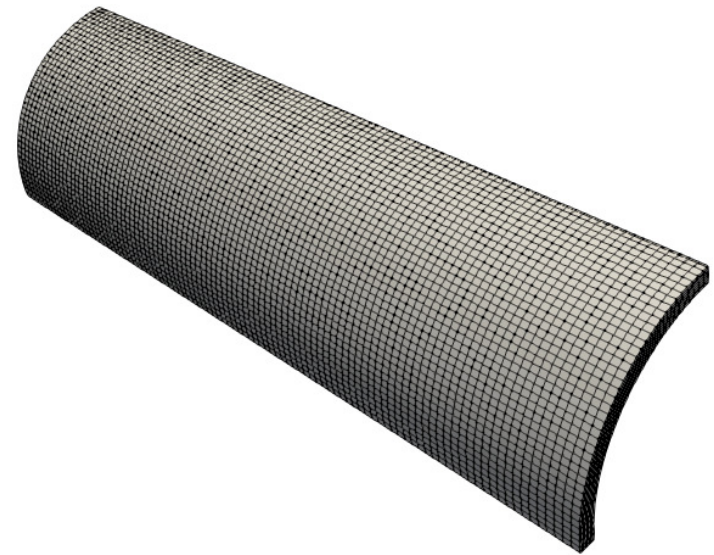

(b) Mesh H2

Figure 22: Dielectric pump: finite element meshes used for the Q1/P0 element. 


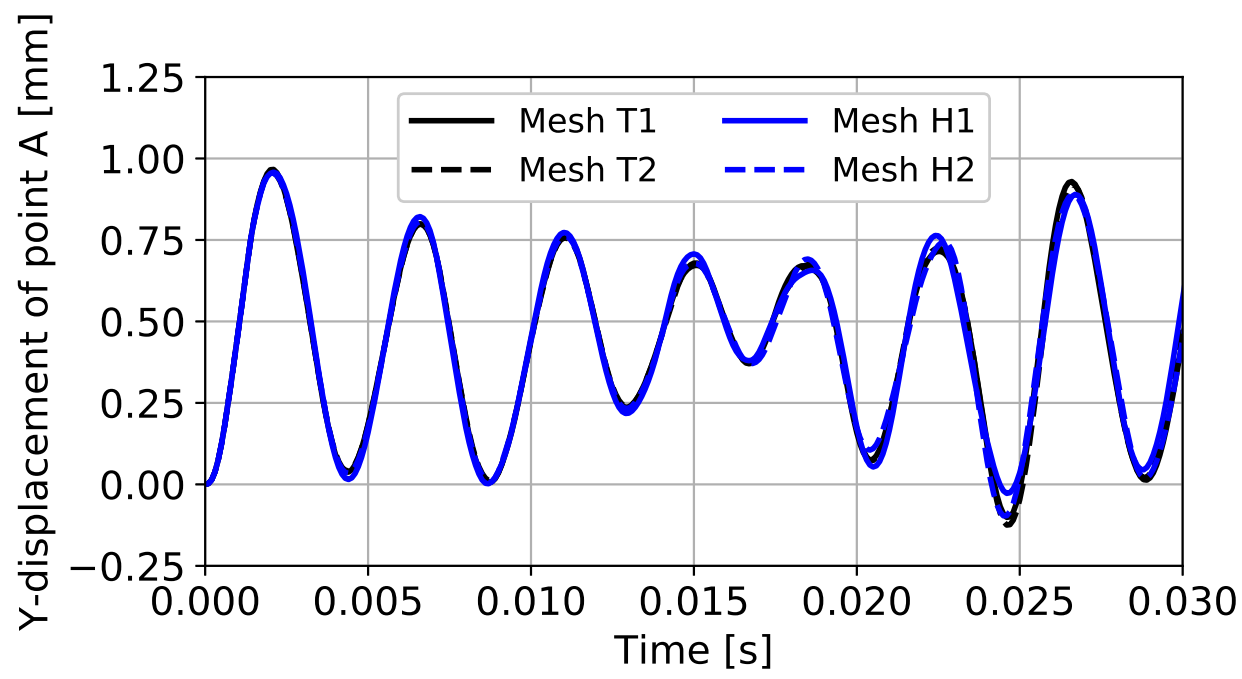

Figure 23: Dielectric pump: the evolution of Y-displacement of point A obtained with different finite element meshes.

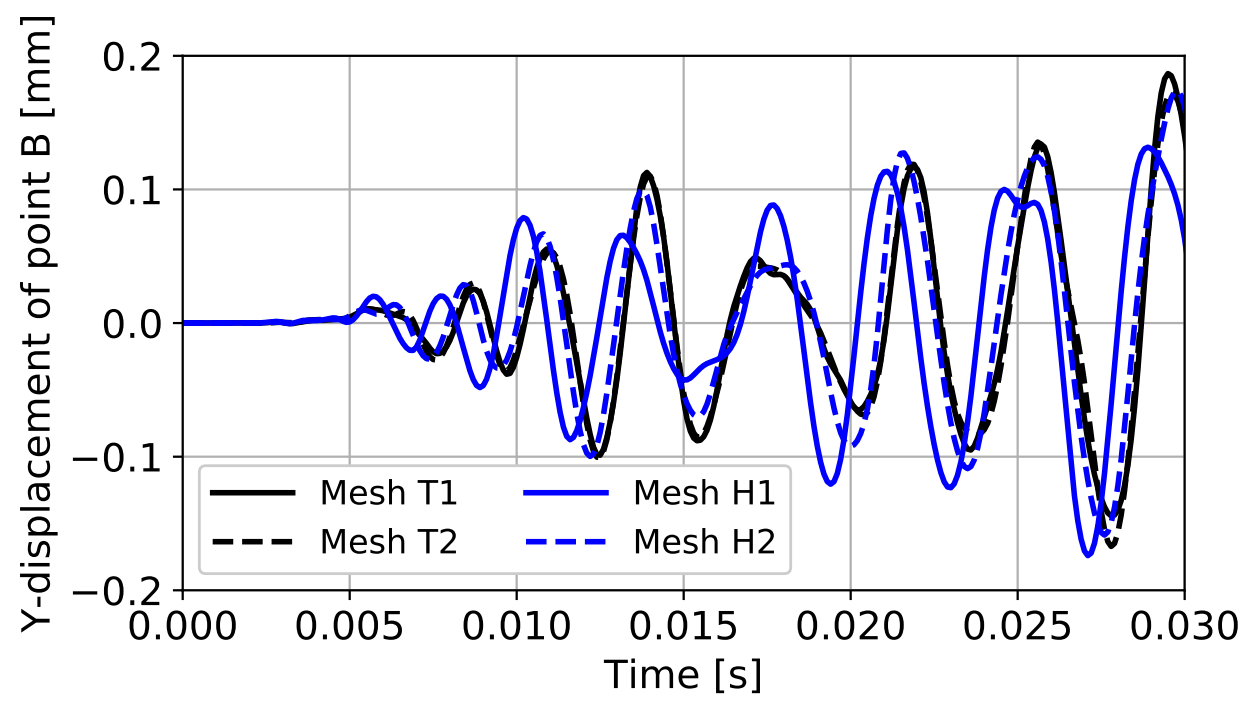

Figure 24: Dielectric pump: the evolution of Y-displacement of point B obtained with different finite element meshes. 


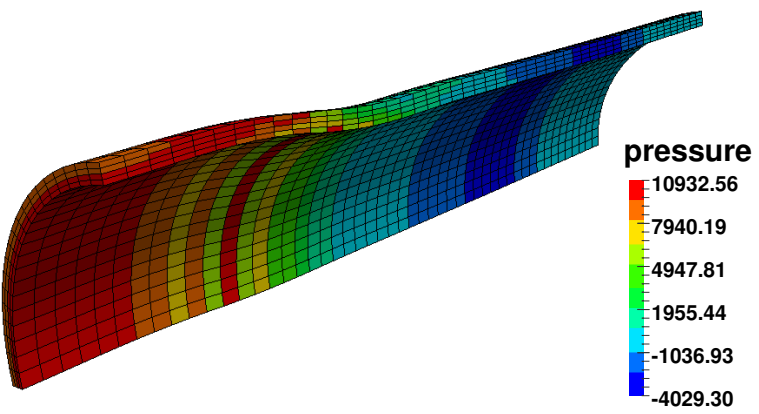

(a) $t=2 \mathrm{~ms}$

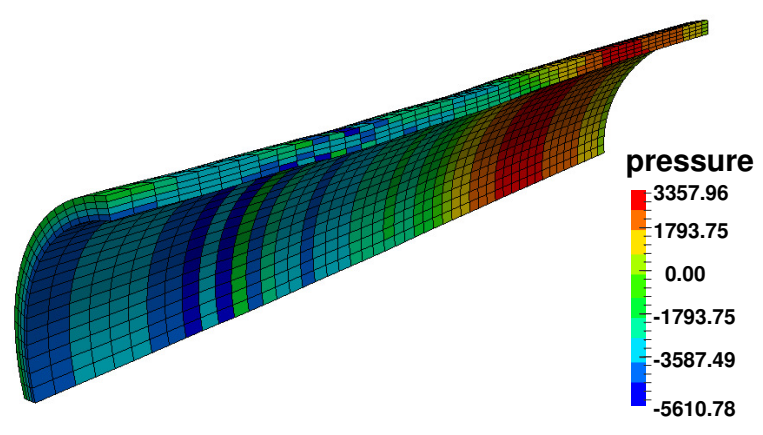

(c) $t=4 \mathrm{~ms}$

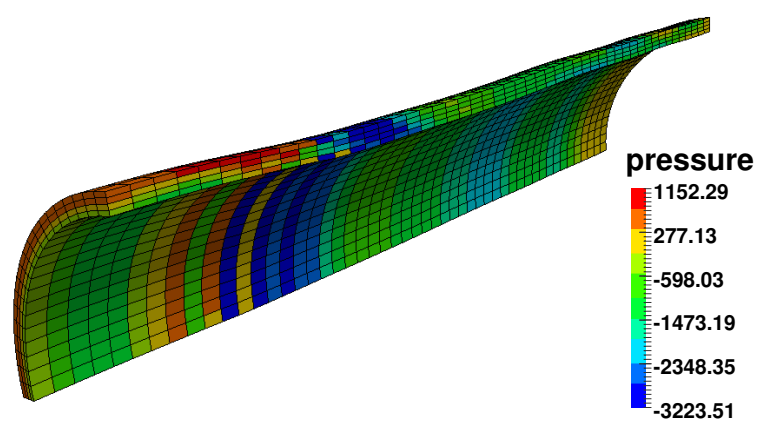

(e) $t=8 \mathrm{~ms}$

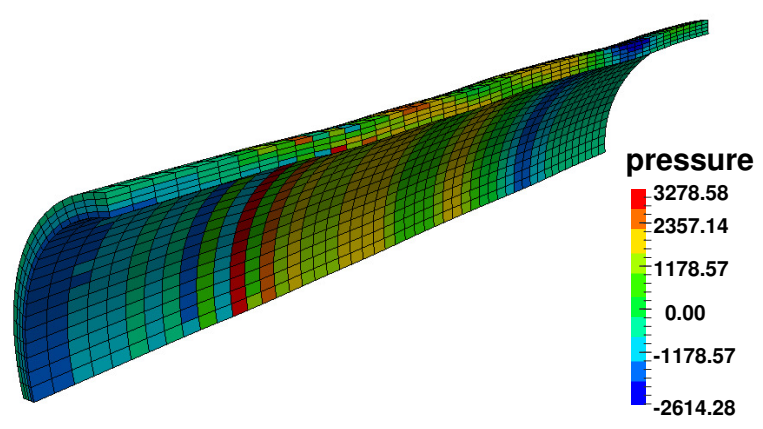

(g) $t=20 \mathrm{~ms}$

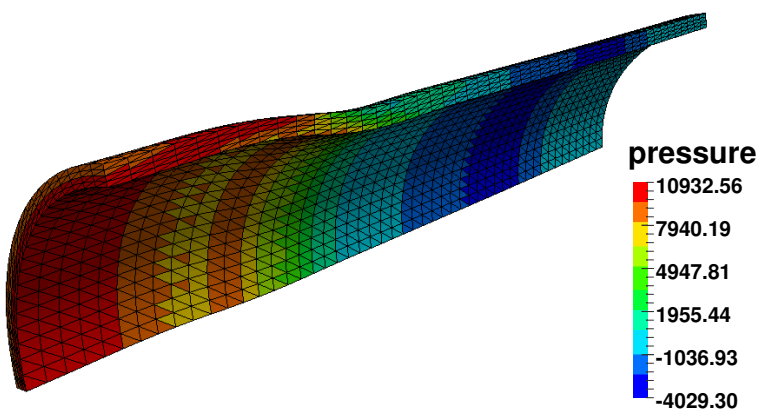

(b) $t=2 \mathrm{~ms}$

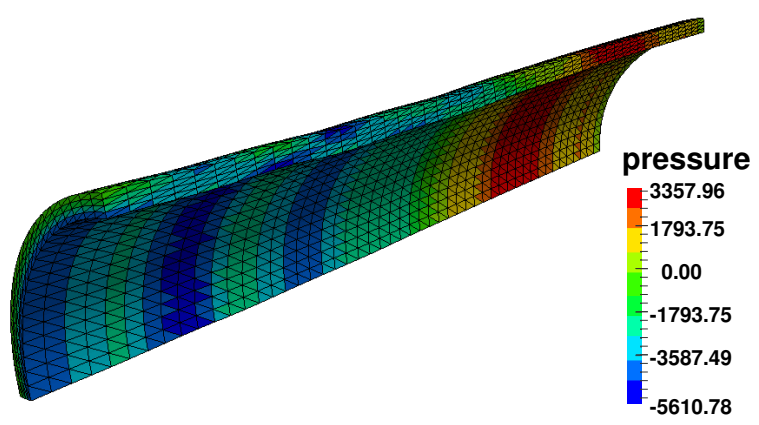

(d) $t=4 \mathrm{~ms}$

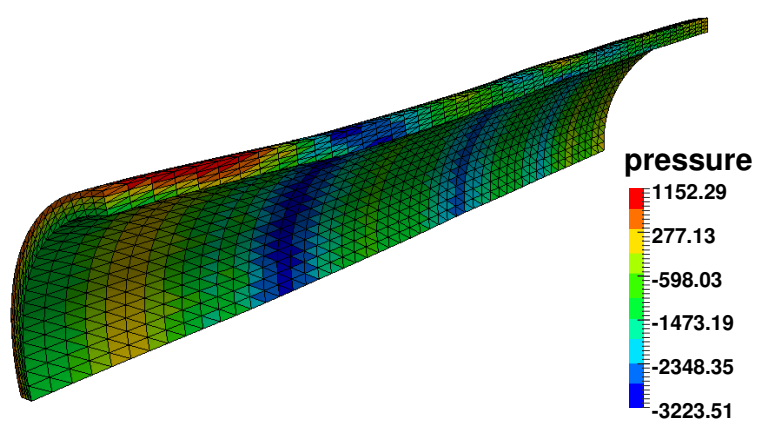

(f) $t=8 \mathrm{~ms}$

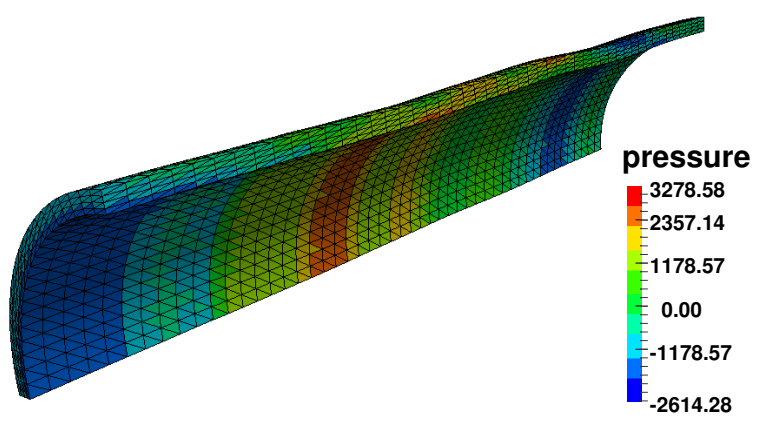

(h) $t=20 \mathrm{~ms}$

Figure 25: Dielectric pump: contour plots of element-wise pressure at four time instants. 


\subsection{Thin helical actuator}

Inspired by the work of $\mathrm{Li}$ et al. [122], this example is concerned with the dynamic simulation of a thin helical actuator. The geometry and boundary conditions of the problem are shown in Fig. 26a. The diameter, pitch and width of the helical strip are $5 \mathrm{~mm}$, and its thickness is $0.2 \mathrm{~mm}$. The finite element mesh used for the analysis shown in Fig. 26b consists of 13789 nodes and 6541 (BT2/BT1/BT2) elements. The material model is assumed to be the truly incompressible Arruda-Boyce model with a shear modulus of $\mu=2.5 \times 10^{4} \mathrm{~Pa}$, number of chain segments, $N=2.8$ and a relative permittivity of $\varepsilon_{r}=10$.

Simulations are performed for a sinusoidally varying voltage $\phi(t)=1-\cos (2.0 \pi f) \mathrm{kV}$, with two frequencies of $f=0.1 \mathrm{~Hz}$ and $f=1.0 \mathrm{~Hz}$, for ten cycles with 100 time steps for each cycle. The displacement response of point A as shown in Fig. 27 illustrates that the response follows the applied voltage for $f=0.1$ $\mathrm{Hz}$ while the response involves a complex behaviour involving higher modes for $f=1.0 \mathrm{~Hz}$. The differences in the deformed shapes of the actuator for the different input frequencies are shown in Fig. 28. Thus, this example demonstrates the applicability of the proposed framework for studying the dynamic response of thin electro-active polymeric actuators under different loading conditions.

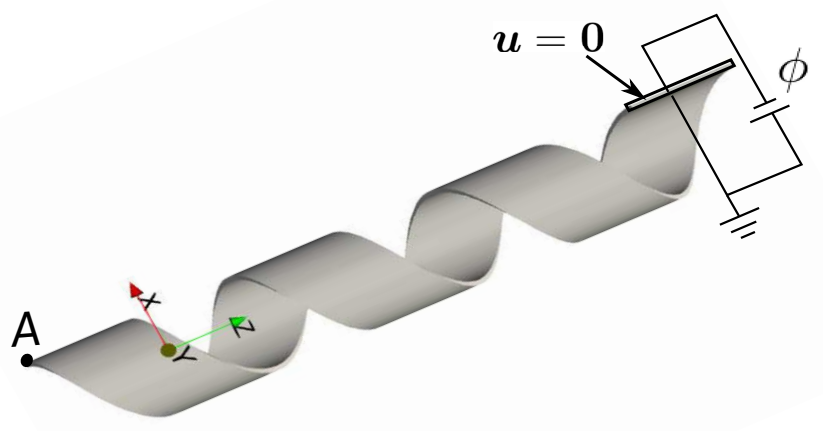

(a)

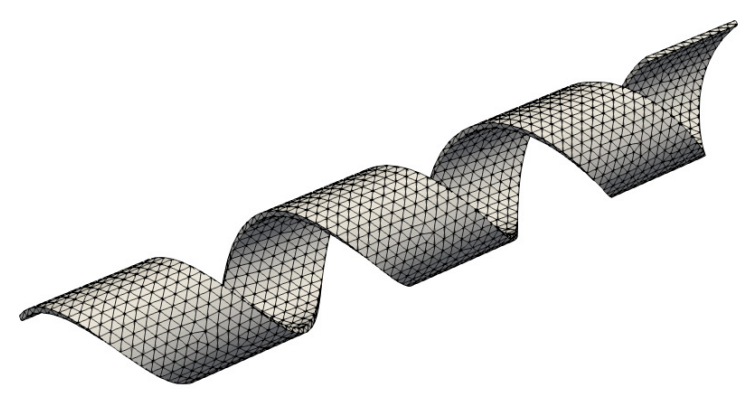

(b)

Figure 26: Helical actuator: (a) geometry and boundary conditions and (b) finite element mesh. Point A is located at the corner of the outer surface.
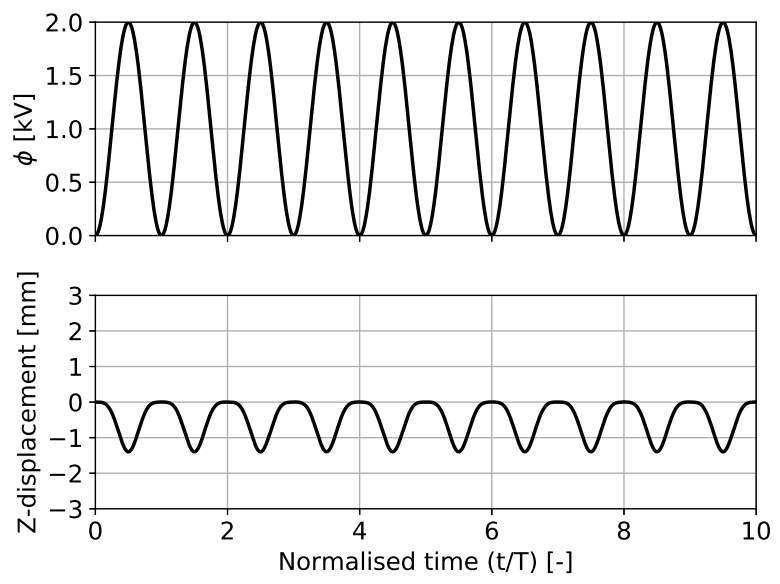

(a) $f=0.1 \mathrm{~Hz}$
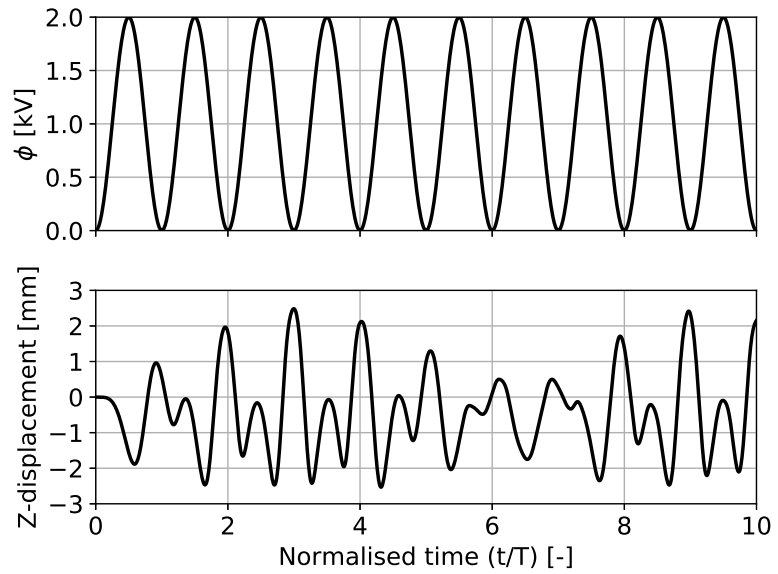

(b) $f=1.0 \mathrm{~Hz}$

Figure 27: Helical actuator: displacement response of point $\mathrm{A}$ for different frequencies. Here, $T(=1 / f) \mathrm{s}$ is the time period. 


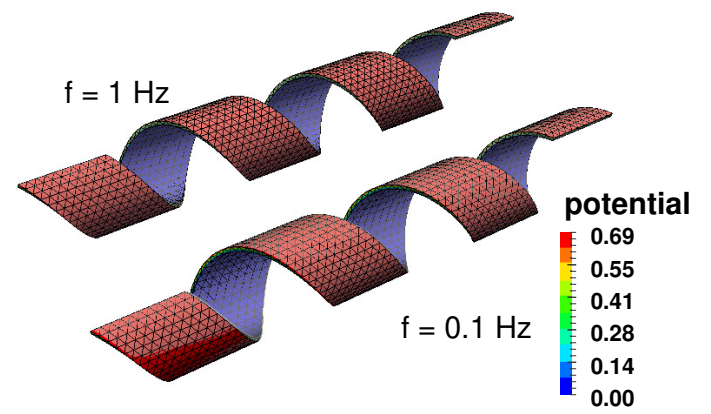

(a) $t / T=0.2$

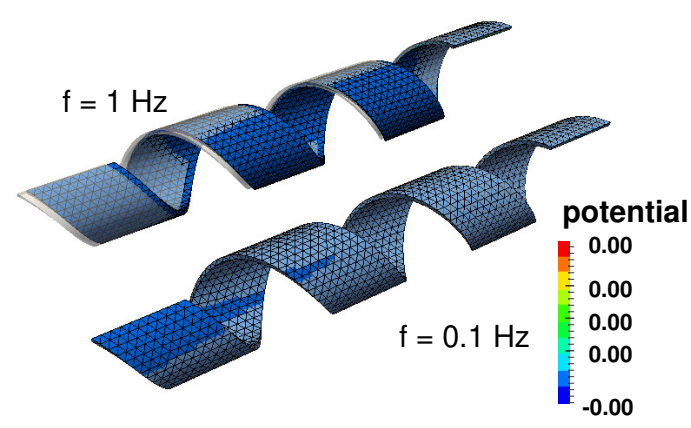

(c) $t / T=1.0$

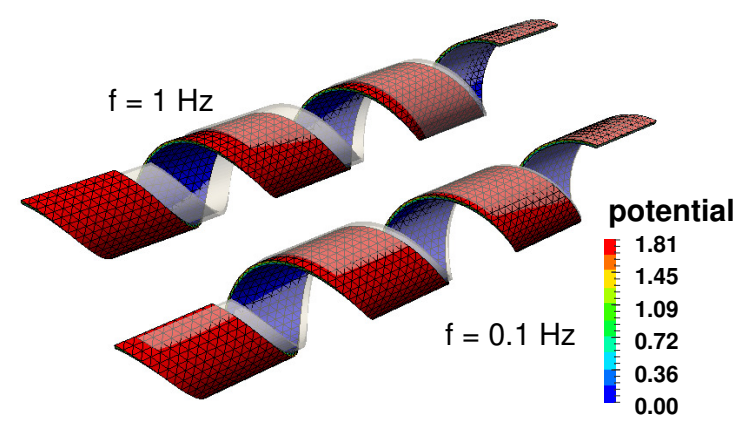

(e) $t / T=1.6$

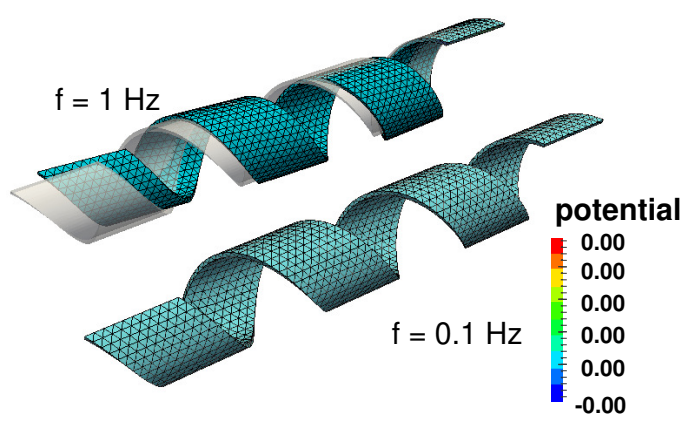

(g) $t / T=2.0$

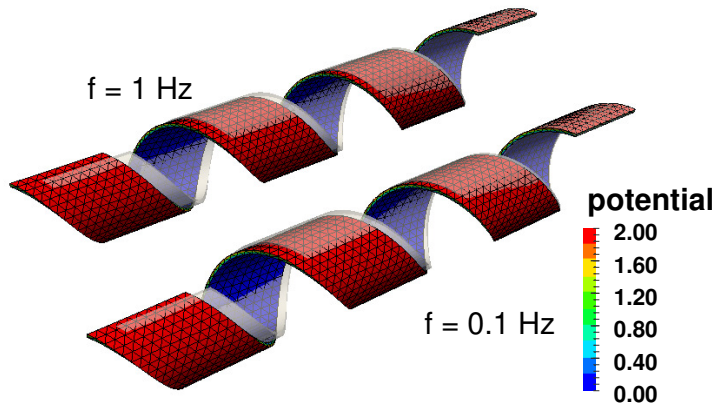

(b) $t / T=0.5$

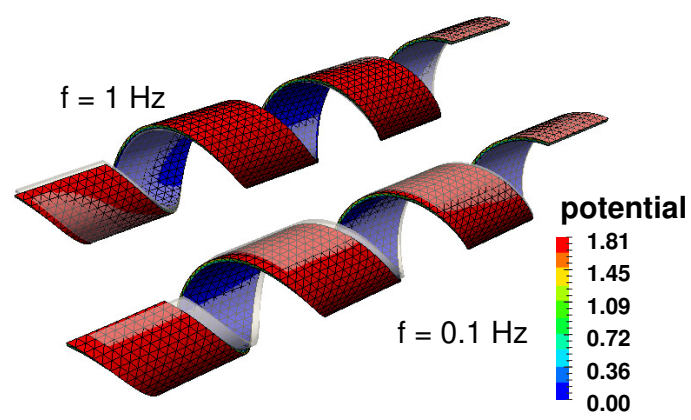

(d) $t / T=1.4$

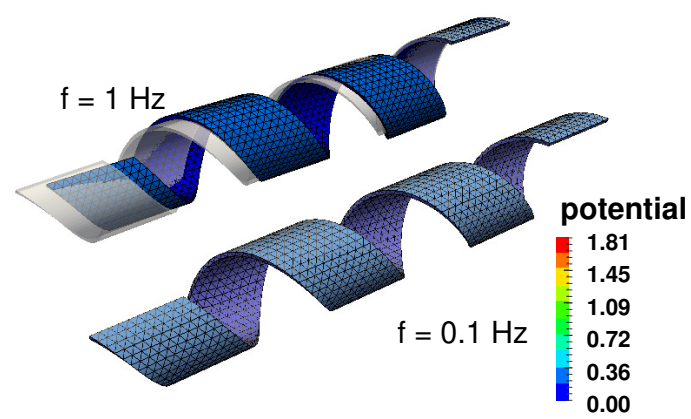

(f) $t / T=1.9$

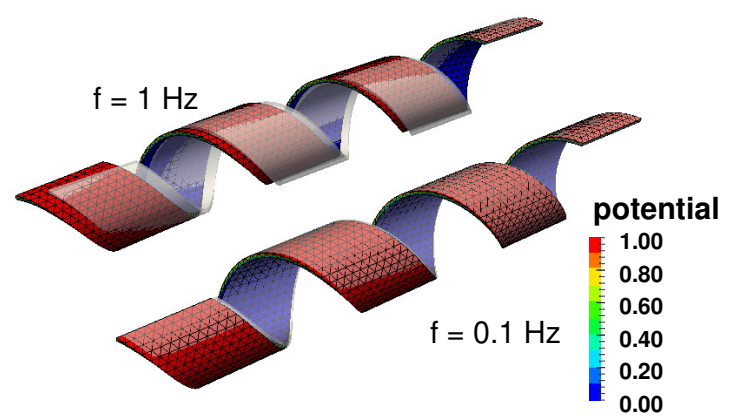

(h) $t / T=2.25$

Figure 28: Helical actuator: deformed shapes of the actuator at eight different time instants. Here, $T(=1 / f) \mathrm{s}$ is the time period.

\subsection{Buckling instabilities in dielectric elastomers}

In the following examples, we demonstrate the ability of the proposed finite element framework to simulate the frequently observed instabilities in dielectric elastomers. For this purpose, we consider a thin semicylindrical shell and thin semi-spherical shell models. The material model is assumed to be the truly incompressible Arruda-Boyce model with a shear modulus, $\mu=10^{4} \mathrm{~Pa}$, number of chain segments, $N=5$ and a 
relative permittivity, $\varepsilon_{r}=5$, and the density of the material is taken as $\rho_{0}=1200 \mathrm{~kg} / \mathrm{m}^{3}$.

\subsubsection{Semi cylindrical shell}

The shell is of length $200 \mathrm{~mm}$, mean radius $20 \mathrm{~mm}$ and thickness $0.5 \mathrm{~mm}$, as shown in Fig. 29. Due to the symmetry of the geometry and boundary conditions, only one-fourth of the domain is considered for the analysis. The quarter portion is discretised with an unstructured finite element mesh consisting of 21005 nodes and 12180 (BT2/BT1/BT2) elements. The mechanical boundary conditions are such that

$$
\begin{array}{rr}
\text { Symmerty BC: } & u_{z}=0 \quad \text { at } \quad Z=-100, \\
\text { Symmerty BC: } & u_{x}=0 \text { at } \quad X=0, \\
\text { Fixed BC: } & \boldsymbol{u}=\mathbf{0} \quad \text { at } \quad X=19.75 .
\end{array}
$$

For the electrical boundary conditions, the inner cylindrical surface is held at a zero voltage while a positive voltage is applied on the outer cylindrical surface. To study the onset of instabilities and the dynamic buckling behaviour, four different values of voltage, 2, 3, 4, $5 \mathrm{kV}$, are considered. The applied voltage is increased smoothly to the maximum specified value over a period of one second and then held constant, as shown in Fig. $30 \mathrm{a}$.

The evolution of Y-displacement of point B for different applied voltage values is shown in Fig. 30b and the deformed shapes of the shell at different time instants are presented in Fig. 31. As shown, the shell does not experience any instabilities for the applied voltage of $2 \mathrm{kV}$ while the instabilities are clearly evident for the other values, with the onset of instability appearing earlier with increasing the value of the applied voltage. The deformed shapes illustrate that the complex deformations in dielectric elastomers can be simulated effectively using very coarse meshes in the proposed framework. This superiority of the proposed scheme is due to the use of higher-order spatial discretisation for the solution variables when compared with that of the Q1/P0 element.

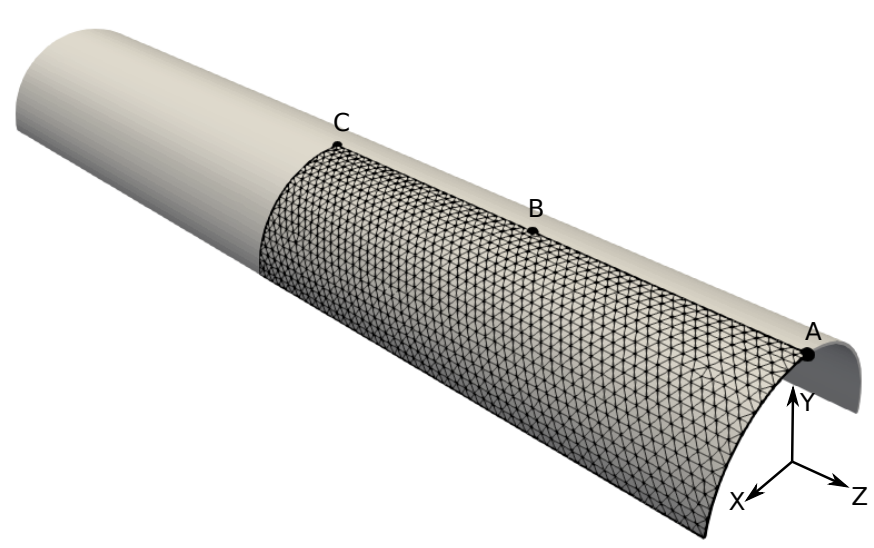

(a)

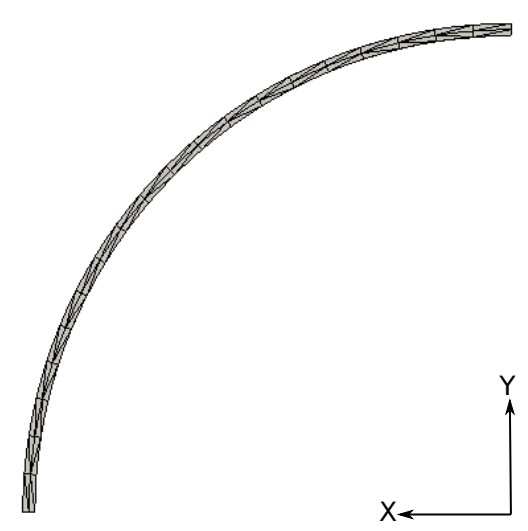

(b)

Figure 29: Cylindrical shell: (a) problem description together with the finite element mesh used for the simulations and (b) side view of the finite element mesh as seen from the positive Z-axis. 


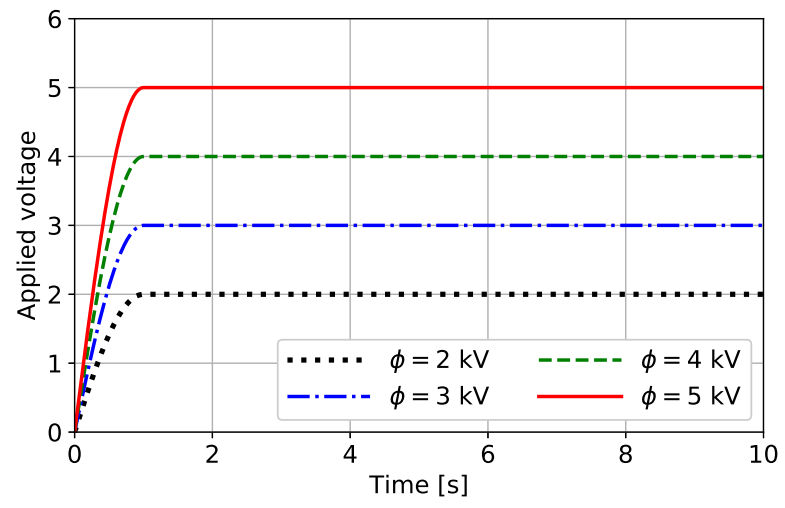

(a)

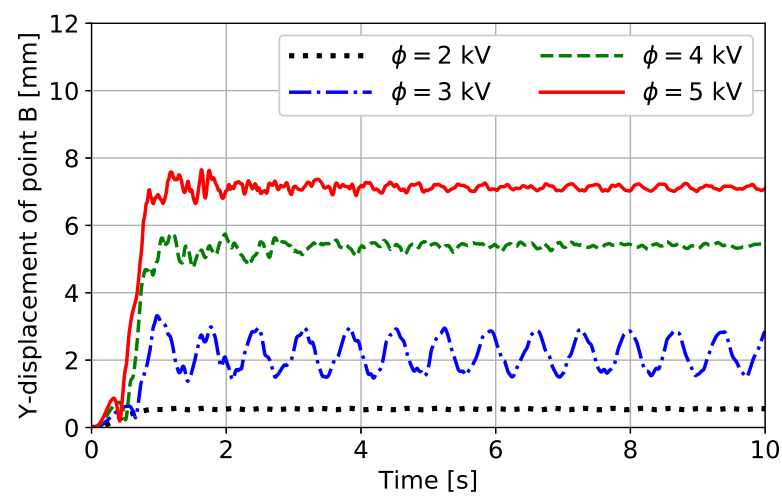

(b)

Figure 30: Cylindrical shell: (a) variation of the applied voltage with respect to time and (b) time evolution of Ydisplacement of point $\mathrm{B}$ for different values of applied voltage.
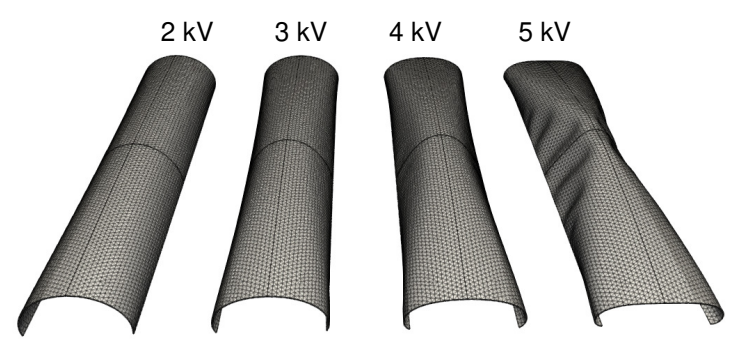

(a) $t=0.4 \mathrm{~s}$
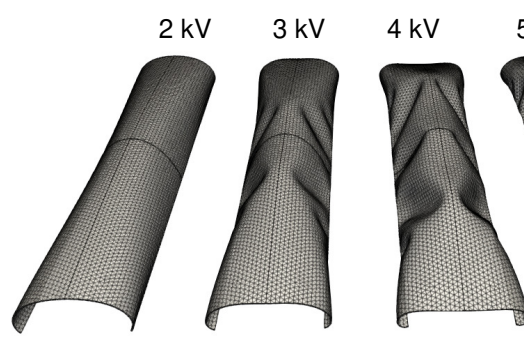

(c) $t=0.8 \mathrm{~s}$
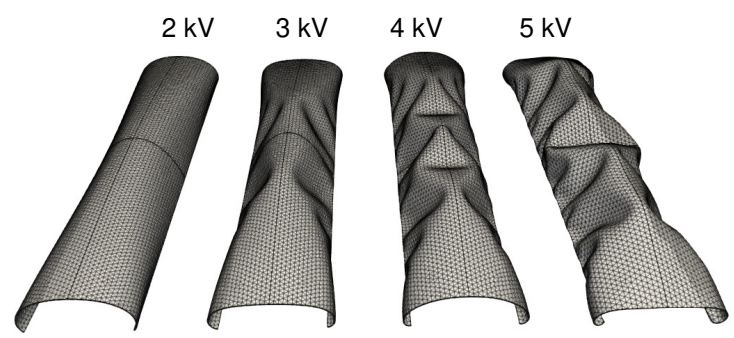

(e) $t=2.0 \mathrm{~s}$
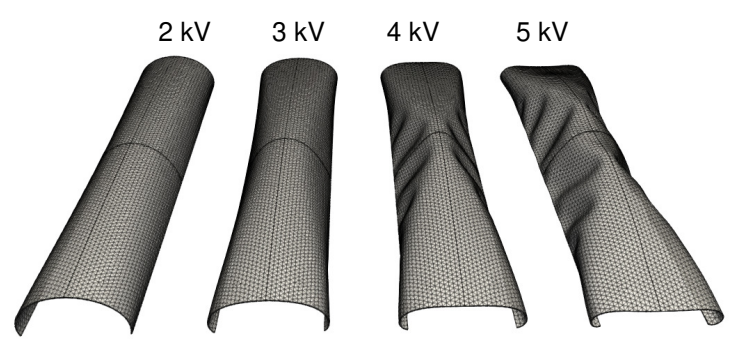

(b) $t=0.5 \mathrm{~s}$
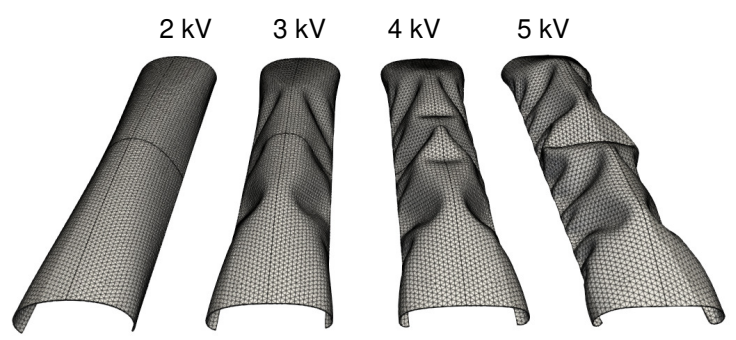

(d) $t=1.0 \mathrm{~s}$
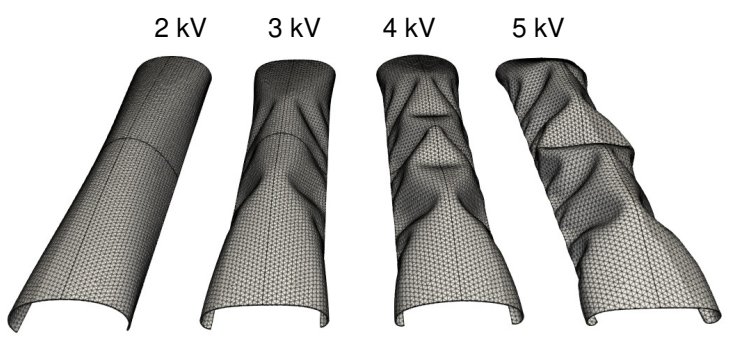

(f) $t=5.0 \mathrm{~s}$

Figure 31: Cylindrical shell: deformed shapes of the shell at different instants for the four different values of applied voltage. 


\subsubsection{Semi spherical shell}

To further demonstrate the superior performance of the proposed framework over other FE frameworks in capturing instabilities in thin polymeric shells, we consider a thin semi-spherical shell of mean radius 20 $\mathrm{mm}$ and thickness $0.5 \mathrm{~mm}$, as shown in Fig. 32. The flat face of the shell is clamped from moving, and a maximum voltage difference of $6 \mathrm{kV}$ is applied across the thickness of the shell. Similar to the previous example, the applied voltage is ramped smoothly to its maximum value over a period of one second and then is held constant. Due to the symmetry boundary conditions, only a half portion is simulated using four finite element meshes displayed in Fig. 33. Meshes T1, T2, H1 and H2 consist of 20225, 77425, 19245, and 75965 nodes, and 11832, 45852, 14992, and 59968 elements. For the Q1/P0 element, four different values of the bulk modulus are chosen to study its effects on the ability of the Q1/P0 element in capturing the instabilities.

Simulations are performed using two different time steps, $\Delta t=0.01$ and $\Delta t=0.005$ seconds. All the simulations crash around 0.75 seconds due to severe mesh distortions. The evolution of X-displacement of highlighted nodes for the T1 and T2 meshes, as shown in Fig. 34, indicate clear spatial and temporal convergences of the proposed scheme before the onset of instabilities. Contour plots of pressure in Fig. 35 demonstrate smooth pressure fields obtained with the proposed element.

The deformed shapes of the shell in Figs. 36 and 37 illustrate that the proposed element with the coarse mesh (T1 mesh) produces exactly the same number of wrinkles as that of the fine mesh (T2 mesh) whereas the Q1/P0 element with a coarse mesh (H1 mesh) fails to produce correct number of wrinkles; only the fine hexahedral mesh (H2 mesh) with the time step of $\Delta t=0.005 \mathrm{~s}$ produces the correct number of wrinkles. Moreover, our effort to ensure the stronger imposition of the incompressibility constraint by using higher values of bulk modulus displays a clear sensitivity of the Q1/P0 element with respect to the bulk modulus, as shown in Figs. 38 and 39.

From these results, it is evident that the proposed scheme produces accurate results using coarse meshes and larger time steps while the Q1/P0 element requires finer meshes and smaller time steps for producing the accurate numerical results, in addition to the fact that the numerical results obtained with the Q1/P0 element are highly sensitive to the bulk modulus. This ability of the proposed framework to produce accurate numerical results using coarse meshes and large steps while still maintaining the exact imposition of the incompressibility makes it an accurate, robust and computationally efficient numerical framework for electromechanics.

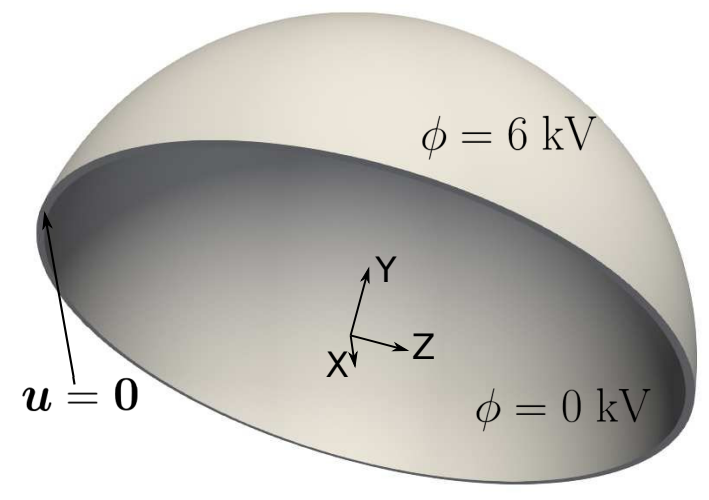

Figure 32: Spherical shell: a schematic description of boundary conditions. 


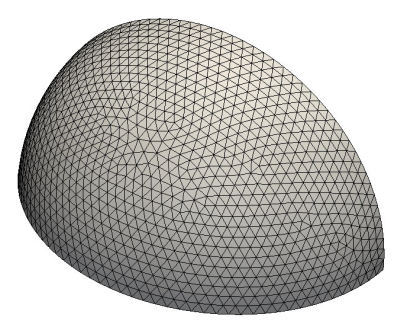

(a) Mesh T1

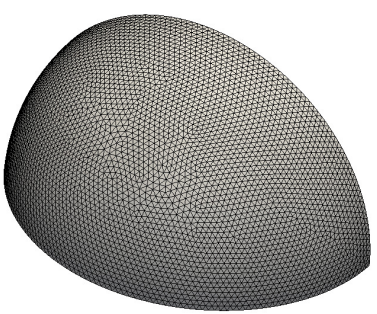

(b) Mesh T2

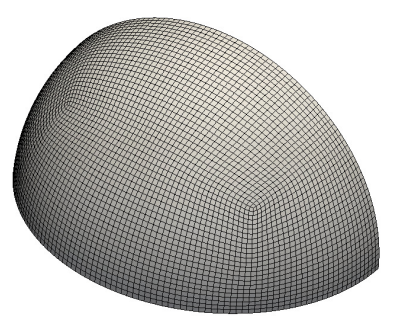

(c) Mesh H1

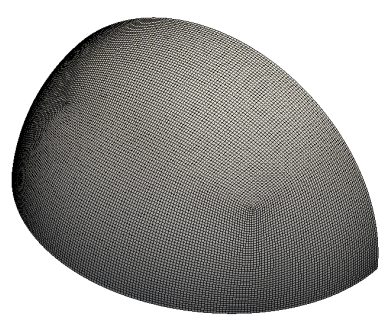

(d) Mesh H2

Figure 33: Spherical shell: finite element meshes used for the analysis.
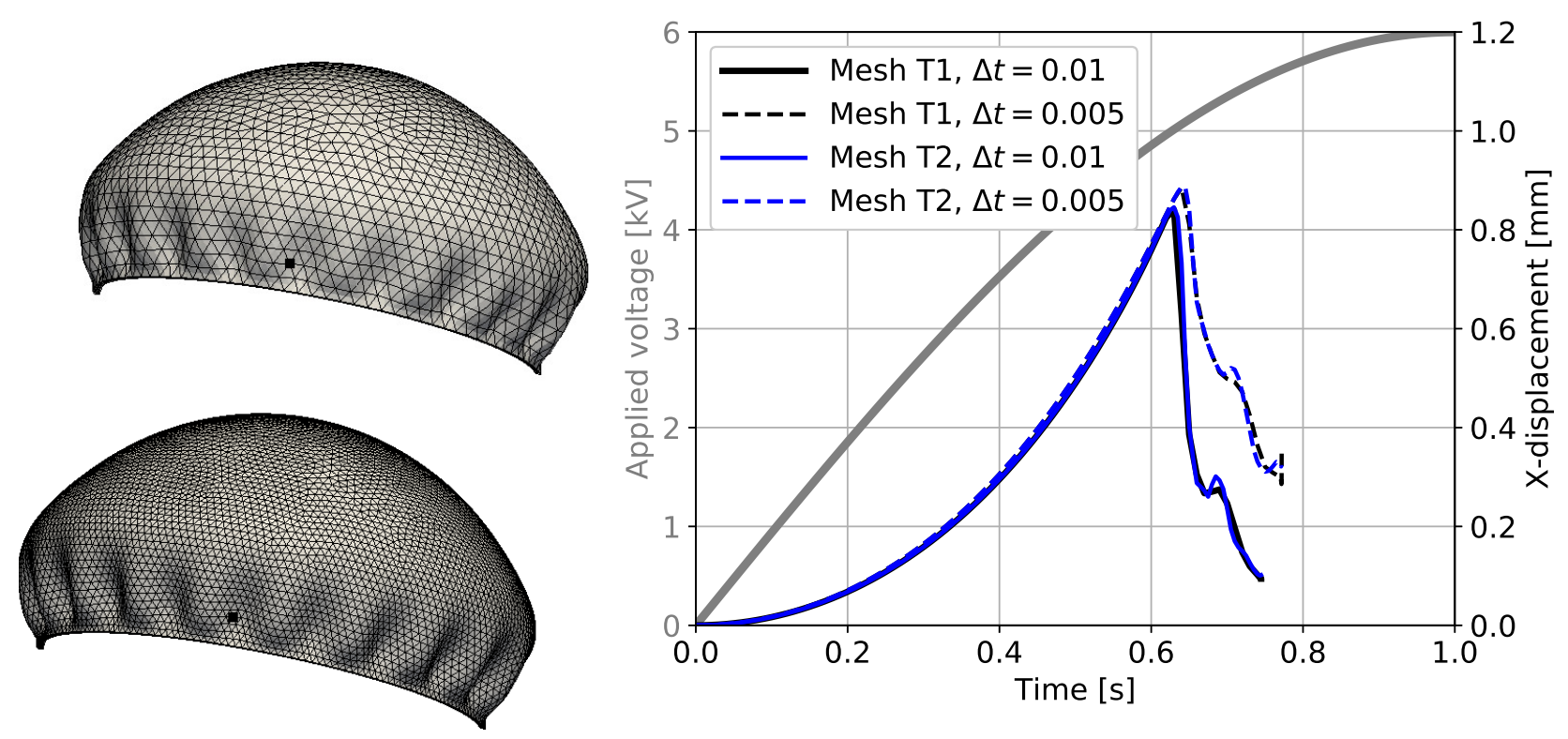

Figure 34: Spherical shell: time evolution of X-displacement of nodes highlighted.

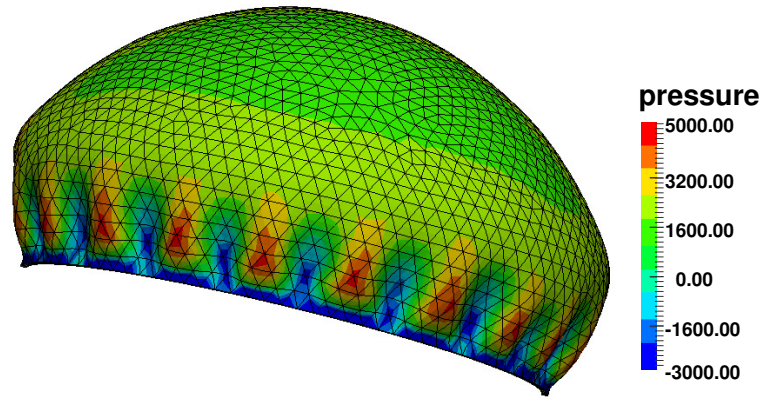

(a) Mesh T1

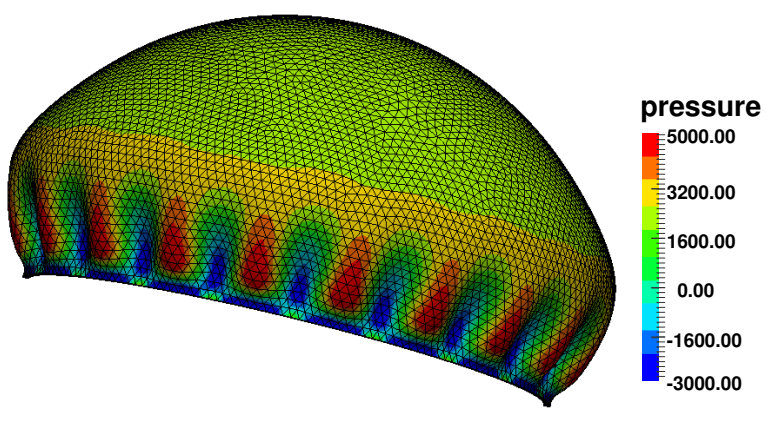

(b) Mesh T2

Figure 35: Spherical shell: contour plots of nodal pressure obtained using the T1 and T2 meshes with a time step of $\Delta t=0.01 \mathrm{~s}$. 


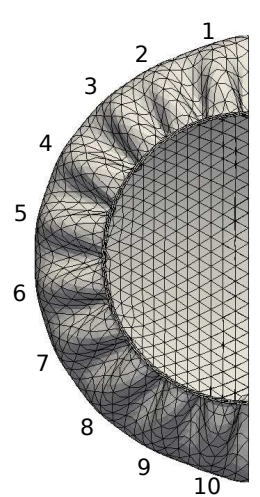

(a) Mesh T1, $\Delta t=0.01 \mathrm{~s}$

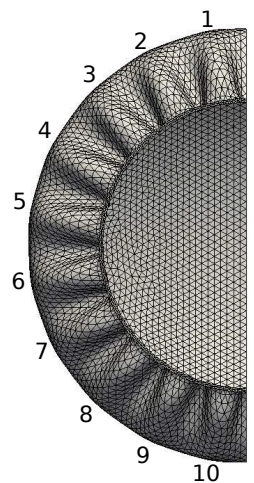

(b) Mesh T2, $\Delta t=0.01 \mathrm{~s}$

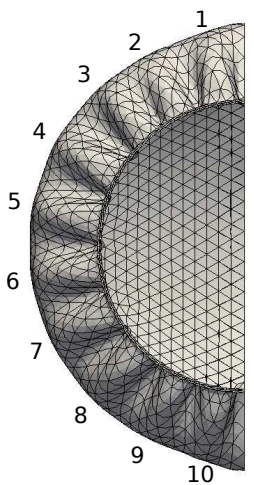

(c) Mesh T1, $\Delta t=0.005 \mathrm{~s}$

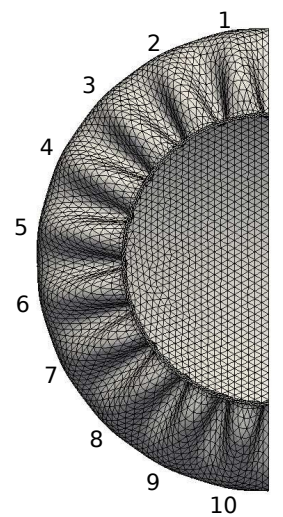

(d) Mesh T2, $\Delta t=0.005 \mathrm{~s}$

Figure 36: Spherical shell: deformed shapes for the T1 and T2 meshes using the proposed scheme. For a better visualisation, the deformation is applied with a warp factor of two.

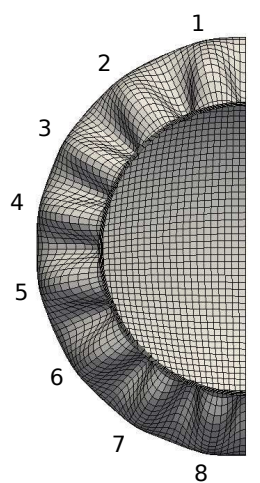

(a) Mesh H1, $\kappa / \mu=10^{3}$, $\Delta t=0.01 \mathrm{~s}$

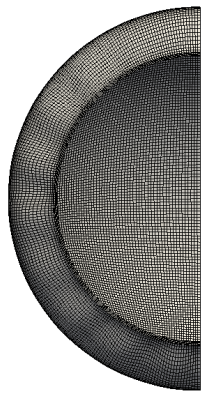

(b) Mesh H2, $\kappa / \mu=10^{3}$, $\Delta t=0.01 \mathrm{~s}$

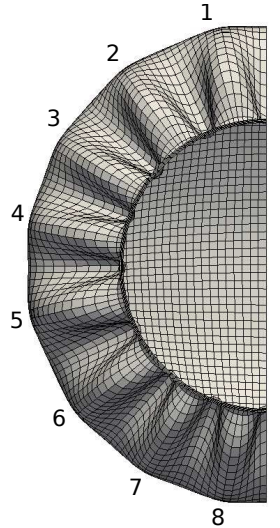

(c) Mesh H1, $\kappa / \mu=10^{3}$, $\Delta t=0.005 \mathrm{~s}$

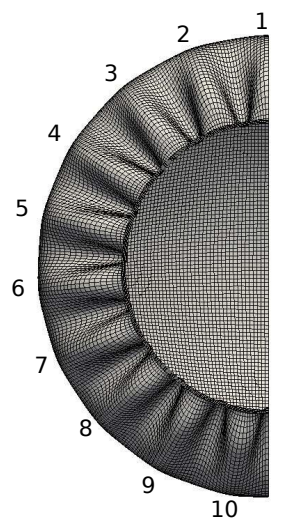

(d) Mesh H2, $\kappa / \mu=10^{3}$, $\Delta t=0.005 \mathrm{~s}$

Figure 37: Spherical shell: deformed shapes for the H1 and H2 meshes using the Q1/P0 element. For a better visualisation, the deformation is applied with a warp factor of two.

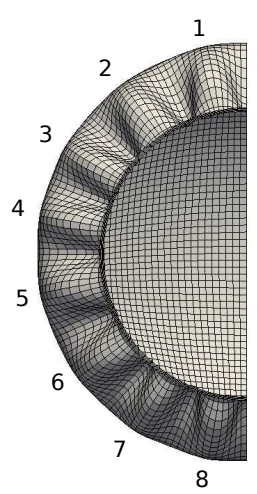
(a) $\kappa / \mu=10^{3}$, $\Delta t=0.01 \mathrm{~s}$
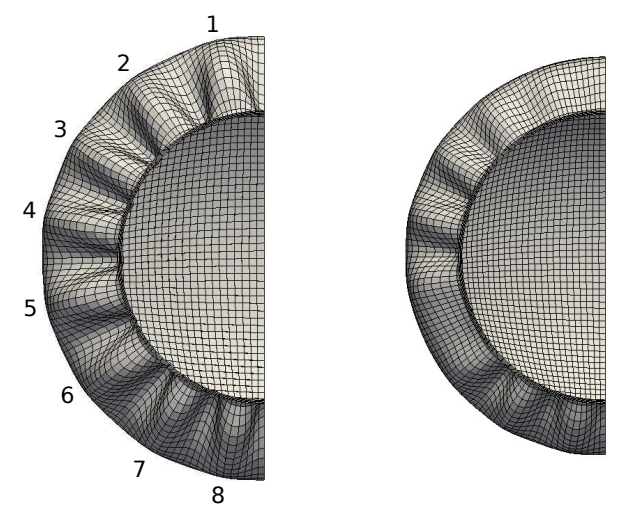

(b) $\kappa / \mu=10^{4}$, $\Delta t=0.01 \mathrm{~s}$ (c) $\kappa / \mu=10^{5}$, $\Delta t=0.01 \mathrm{~s}$

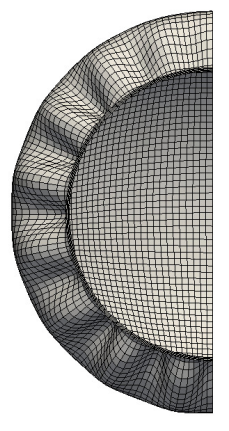

(d) $\kappa / \mu=10^{6}$, $\Delta t=0.01 \mathrm{~s}$

Figure 38: Spherical shell: deformed shapes for the H1 mesh using the Q1/P0 element with different values of the bulk modulus. For a better visualisation, the deformation is applied with a warp factor of two. 


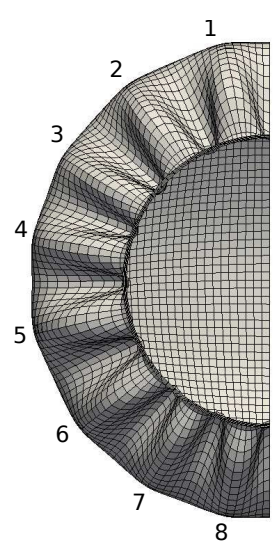

(a) $\kappa / \mu=10^{3}$, $\Delta t=0.005 \mathrm{~s}$

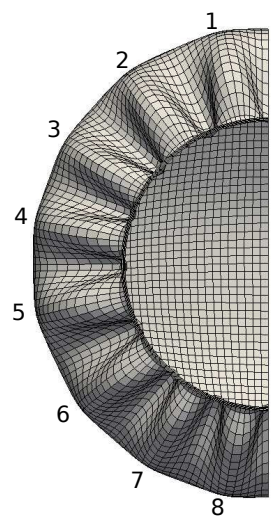

(b) $\kappa / \mu=10^{4}$, $\Delta t=0.005 \mathrm{~s}$

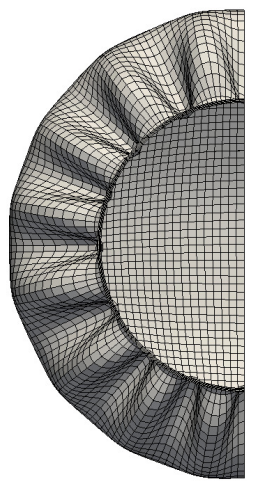

(c) $\kappa / \mu=10^{5}$, $\Delta t=0.005 \mathrm{~s}$

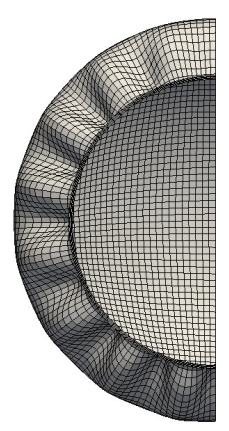

(d) $\kappa / \mu=10^{6}$, $\Delta t=0.005 \mathrm{~s}$

Figure 39: Spherical shell: deformed shapes for the H1 mesh using the Q1/P0 element using different values of bulk modulus. For a better visualisation, the deformation is applied with a warp factor of two.

\section{Summary and conclusion}

In this contribution, we have proposed a novel finite element framework for computational electromechanics at finite strains. The novel contributions of the present work are the extension of a recently published two-field mixed displacement-pressure formulation to coupled electromechanics and the use of higher-order Bézier and Lagrange elements for the spatial discretisation. The proposed elements are named as BT2/BT1/BT2, P2/P1/P2 and $\mathrm{Q} 2 / \mathrm{Q} 1 / \mathrm{Q} 2$ for the ease of reference.

First, the accuracy of the proposed formulation and its ability to compute accurate numerical solutions using coarse meshes is demonstrated by studying the examples of thin bi-layer beams in two and three dimensions. It is observed that, among all the elements considered in this work, the Q2/Q1/Q2 element produces the best quality results for the coarsest mesh and that the differences between the results obtained with higher-order elements match well each other after one refinement. Then, the computational advantages of the proposed framework over the Q1/P0 and Q1-F-bar elements are illustrated with the examples of a spherical gripper. Later, its capability in computing accurate numerical solutions for elastodynamics problems using coarse meshes is proven with the example of a dielectric pump. Finally, the ability of the novel finite element framework to effectively simulate instabilities in dielectric elastomers is illustrated with the examples of thin semi-cylindrical and semi-spherical shells.

The important characteristics of the proposed finite element framework are:

- Computational efficiency: The computational efficiency of the proposed framework results from the combination of (a) two-field mixed formulation for the mechanical problem, (b) quadratic polynomials for the displacement and electric potential, and a linear approximation for the pressure, (c) ability to compute accurate numerical solutions using coarse meshes and (d) ability to complete the simulations using fewer load steps in elastostatic problems and fewer time steps in elastodynamics problems.

- Robustness: The robustness of the proposed framework is due to the fact that it employs inf-sup stable elements, as opposed to the Q1/P0 element, which is inf-sup unstable. Moreover, unlike the Q1/P0 element, the convergence of iterations with the proposed element is not sensitive to changes in the value of the bulk modulus.

- Complex geometries: The use of triangular and tetrahedral elements significantly eases the task of mesh generation for the proposed framework, unlike the schemes based on the quadrilateral and hexahedral elements for which mesh generation for complex geometries is quite cumbersome.

To conclude, the demonstrated advantages of the proposed framework prove that it is a very promising finite element framework for the simulation of complex coupled interactions in electro-active polymers. Fundamental computational advantages of this novel framework can be extended to develop robust, accurate, and computationally efficient computational tools for the simulation of complex multiphysics phenomena of thermoelectro-mechanical interactions as well as coupled fluid-structure interactions in electromechanical systems 
for applications in biomedical engineering and energy harvesting. In forthcoming contributions, we will extend this framework to capture visco-anisotropic as well as coupled thermo-electro-mechanical behaviour of electro-active polymers.

\section{ACKNOWLEDGEMENTS}

The first author acknowledges the support of the Supercomputing Wales project, which is part-funded by the European Regional Development Fund (ERDF) via the Welsh Government.

\section{Appendix A. First and second variations of the energy functions}

The first variation of the strain energy function $(\Psi)$ and the perturbed Lagrangian $\left(\Psi_{\mathrm{PL}}\right)$ is

$$
\delta\left(\Psi+\Psi_{\mathrm{PL}}\right)=\delta F_{i J} \bar{P}_{i J}-\delta \mathbb{E}_{I} \mathbb{D}_{I}+\delta J p+\delta p\left[J-1-\frac{p}{\kappa}\right]
$$

where

$$
\begin{aligned}
\bar{P}_{i J} & =\frac{\partial \Psi}{\partial F_{i J}} \\
\mathbb{D}_{I} & =-\frac{\partial \Psi}{\partial \mathbb{E}_{I}}
\end{aligned}
$$

Using,

$$
\begin{aligned}
\delta F_{i J} & =\delta u_{i, J}=\delta u_{i, j} F_{j J} \\
\delta J & =J \delta u_{i, i}=J \delta u_{i, j} \delta_{i j} \\
\mathbb{D}_{I} & =J F_{I i}^{-1} \mathbb{d}_{i} \\
\delta \mathbb{E}_{I} & =F_{i I} \delta \mathbb{e}_{i} \\
\bar{P}_{i J} & =F_{i I} \bar{S}_{I J} \\
\delta \mathbb{e}_{i} & =-\delta \phi_{, i}
\end{aligned}
$$

in (A.1) we obtain

$$
\begin{aligned}
\delta\left(\Psi+\Psi_{\mathrm{PL}}\right) & =\delta u_{i, j} J \bar{\sigma}_{i j}+\delta \phi_{, i} J \mathbb{d}_{i}+\delta u_{i, j} \delta_{i j} p J+\delta p\left[J-1-\frac{p}{\kappa}\right] \\
& =\delta u_{i, j} J \widehat{\sigma}_{i j}+\delta \phi_{, i} J \mathbb{d}_{i}+\delta p\left[J-1-\frac{p}{\kappa}\right]
\end{aligned}
$$

where $\delta_{i j}$ is the Kronecker delta and $\widehat{\sigma}_{i j}$ is the effective Cauchy stress tensor for the mixed formulation. $\widehat{\sigma}_{i j}$ is given by

$$
\widehat{\sigma}_{i j}=\bar{\sigma}_{i j}+p \delta_{i j}
$$

Now, the second variation of the energy functions is computed as the variation of (A.1) as,

$$
\begin{aligned}
d(\delta \Psi) & =\delta F_{i J}\left[\frac{\partial \bar{P}_{i J}}{\partial F_{k L}} d F_{k L}+\frac{\partial \bar{P}_{i J}}{\partial \mathbb{E}_{K}} d \mathbb{E}_{K}\right]-\delta \mathbb{E}_{I}\left[\frac{\partial \mathbb{D}_{I}}{\partial F_{j K}} d F_{j K}+\frac{\partial \mathbb{D}_{I}}{\partial \mathbb{E}_{J}} d \mathbb{E}_{J}\right] \\
& +d(\delta J) p+\delta J d p+\delta p d J-\frac{1}{\kappa} \delta p d p \\
& =\delta u_{i, j}\left(J \mathrm{e}_{i j k l}\right) d u_{k, l}+\delta u_{i, j}\left(J \mathrm{p}_{i j k}\right) d \phi_{, k}+\delta \phi_{, i}\left(J \widehat{\mathrm{p}}_{i j k}\right) d u_{j, k}+\delta \phi_{, i}\left(J \mathrm{~d}_{i j}\right) d \phi_{, j} \\
& +\delta u_{i, i} d p J+\delta p d u_{k, k} J-\frac{1}{\kappa} \delta p d p
\end{aligned}
$$

where $d(\bullet)$ denotes another variation of the variable $(\bullet), \mathrm{e}_{i j k l}$ is the material tangent tensor of order four; $\mathrm{p}_{i j k}$ and $\widehat{\mathrm{p}}_{i j k}$ are third-order coupling tensors; and $\mathrm{d}_{i j}$ is the electric permittivity tensor of order two, and they are given by 


$$
\begin{aligned}
\mathrm{e}_{i j k l} & =\frac{1}{J} F_{j J} \frac{\partial \bar{P}_{i J}}{\partial F_{k L}} F_{l L}+p\left[\delta_{i j} \delta_{l k}-\delta_{i l} \delta_{j k}\right] \\
\mathrm{p}_{i j k} & =-\frac{1}{J} F_{j J} \frac{\partial \bar{P}_{i J}}{\partial \mathbb{E}_{K}} F_{k K} \\
\widehat{\mathrm{p}}_{i j k} & =\frac{1}{J} F_{i I} \frac{\partial \mathbb{D}_{I}}{\partial F_{j K}} F_{k K} \\
\mathrm{~d}_{i j} & =-\frac{1}{J} F_{i I} \frac{\partial \mathbb{D}_{I}}{\partial \mathbb{E}_{J}} F_{j J}
\end{aligned}
$$

\section{Appendix B. Index notation for gradient, divergence, strain and stress}

The widely-used Voigt notation for transforming the strain measures, stress measures and tangent tensors into vector and matrix forms leads to complications in transforming the fourth- and third-order tensors resulting from the electrical and coupled energy functions. For the ease of computer implementation of the finite element formulation for electromechanics, in this work, we use the full strain and stress tensors as vectors. Accordingly, the Cauchy stress tensor is represented as a column vector as,

$$
\begin{aligned}
\boldsymbol{\sigma}=\sigma_{i j} & =\left[\begin{array}{lllllllll}
\sigma_{x x} & \sigma_{y x} & \sigma_{z x} & \sigma_{x y} & \sigma_{y y} & \sigma_{z y} & \sigma_{x z} & \sigma_{y z} & \sigma_{z z}
\end{array}\right]^{\mathrm{T}} \\
& =\left[\begin{array}{lllllllll}
\sigma_{11} & \sigma_{21} & \sigma_{31} & \sigma_{12} & \sigma_{22} & \sigma_{32} & \sigma_{13} & \sigma_{23} & \sigma_{33}
\end{array}\right]^{\mathrm{T}} .
\end{aligned}
$$

Analogously, the gradient of the displacement field is represented as

$$
\begin{aligned}
\nabla \boldsymbol{u}=u_{i, j} & =\left[\begin{array}{lllllllll}
u_{x, x} & u_{y, x} & u_{z, x} & u_{x, y} & u_{y, y} & u_{z, y} & u_{x, z} & u_{y, z} & u_{z, z}
\end{array}\right]^{\mathrm{T}} \\
& =\left[\begin{array}{lllllllll}
\frac{\partial u_{x}}{\partial x} & \frac{\partial u_{y}}{\partial x} & \frac{\partial u_{z}}{\partial x} & \frac{\partial u_{x}}{\partial y} & \frac{\partial u_{y}}{\partial y} & \frac{\partial u_{z}}{\partial y} & \frac{\partial u_{x}}{\partial z} & \frac{\partial u_{y}}{\partial z} & \frac{\partial u_{z}}{\partial z}
\end{array}\right]^{\mathrm{T}} \\
& =\mathbf{G}_{\boldsymbol{u}} \mathbf{u}
\end{aligned}
$$

where $\mathbf{G}_{\boldsymbol{u}}$ is the gradient-displacement matrix which, for a single basis function, is given as

$$
\mathbf{G}_{\boldsymbol{u}}=\left[\begin{array}{ccccccccc}
\frac{\partial N_{u}}{\partial x} & 0 & 0 & \frac{\partial N_{u}}{\partial y} & 0 & 0 & \frac{\partial N_{u}}{\partial z} & 0 & 0 \\
0 & \frac{\partial N_{u}}{\partial x} & 0 & 0 & \frac{\partial N_{u}}{\partial y} & 0 & 0 & \frac{\partial N_{u}}{\partial z} & 0 \\
0 & 0 & \frac{\partial N_{u}}{\partial x} & 0 & 0 & \frac{\partial N_{u}}{\partial y} & 0 & 0 & \frac{\partial N_{u}}{\partial z}
\end{array}\right]^{\mathrm{T}}
$$

Similarly, the gradient of the electric potential in the current configuration is computed as

$$
\nabla \phi=\phi_{, i}=\left\{\begin{array}{lll}
\frac{\partial \phi}{\partial x} & \frac{\partial \phi}{\partial y} & \frac{\partial \phi}{\partial z}
\end{array}\right\}^{\mathrm{T}}=\mathbf{G}_{\phi} \boldsymbol{\phi}
$$

with

$$
\mathbf{G}_{\phi}=\left[\begin{array}{lll}
\frac{\partial N_{\phi}}{\partial x} & \frac{\partial N_{\phi}}{\partial y} & \frac{\partial N_{\phi}}{\partial z}
\end{array}\right]^{\mathrm{T}} .
$$

For the derivation of stiffness matrices in this work, we introduce a divergence-displacement matrix, $\mathbf{D}_{\boldsymbol{u}}$, for representing the divergence of the displacement in the vector form as

$$
\nabla \cdot \boldsymbol{u}=u_{i, i}=\frac{\partial u_{x}}{\partial x}+\frac{\partial u_{y}}{\partial y}+\frac{\partial u_{z}}{\partial z}=\mathbf{D}_{\boldsymbol{u}} \mathbf{u}
$$

with $\mathbf{D}_{\boldsymbol{u}}$ for a single basis function as given by

$$
\mathbf{D}_{\boldsymbol{u}}=\left[\begin{array}{lll}
\frac{\partial N_{u}}{\partial x} & \frac{\partial N_{u}}{\partial y} & \frac{\partial N_{u}}{\partial z}
\end{array}\right] .
$$




\section{References}

[1] A. Ask, A. Menzel, M. Ristinma, Phenomenological modeling of viscous electrostrictive polymers, International Journal of Non-Linear Mechanics, 47(2):156-165, 2012.

[2] A. Ask, R. Denzer, A. Menzel, M. Ristinma, Inverse motion-based form finding for quasiincompressible finite electroelasticity, International Journal for Numerical Methods in Engineering, 94(6):554-572, 2013.

[3] D. Ahmad, K. Patra, Experimental and theoretical analysis of laterally pre-stretched pure shear deformation of dielectric elastomer, Polymer Testing, 75: 291-297, 2019.

[4] R. Brighenti, A. Menzel, F. J. Vernerey, A physics-based micromechanical model for electroactive viscoelastic polymers, Journal of Intelligent Material Systems and Structures, 29(14): 2902-2918, 2018.

[5] Y. Bar-Cohen, Electroactive polymer (EAP) actuators as artificial muscles: reality, potential, and challenges, SPIE press, 136, 2004.

[6] P. Brochu, Q. Pei, Advances in dielectric elastomers for actuators and artificial muscles, Macromolecular Rapid Communications, 31(1):10-36, 2010.

[7] D. Bishara, M. Jabareen, A reduced mixed finite-element formulation for modeling the viscoelastic response of electro-active polymers at finite deformation, Mathematics and Mechanics of Solids, 24(5):1578-1610, 2019.

[8] R. Bustamante, Transversely isotropic nonlinear electro-active elastomers, Acta Mechanica, 206(34):237-259, 2009.

[9] R. Bustamante, J. Merodio, Constitutive structure in coupled non-linear electro-elasticity: Invariants descriptions and constitutive restrictions, International Journal of Non-Linear Mechanics, 46:1315-1323, 2011.

[10] R. Bustamante, A variational formulation for a boundary value problem considering an electrosensitive elastomer interacting with two bodies, Mechanics Research Communication, 36(7):791-795, 2009.

[11] R. Bustamante, M. H. B.M. Shariff, New sets of spectral invariants for electro-elastic bodies with one and two families of fibres, European Journal of Mechanics-A/Solids, 58:42-53, 2016.

[12] A. Bueschel, S. Klinkel, W. Wagner, Dielectric elastomers-numerical modeling of nonlinear viscoelectroelasticity, International Journal for Numerical Methods in Engineering, 93(8): 834-856, 2013.

[13] X. Chen, On magneto-thermo-viscoelastic deformation and fracture, International Journal of NonLinear Mechanics, 44:244-248, 2009.

[14] F. Carpi, D. D. Rossi, Improvement of electromechanical actuating performances of a silicone dielectric elastomer by dispersion of titanium dioxide powder, IEEE Transactions on Dielectrics and Electrical Insulation, 12(4): 835-843, 2005.

[15] N. Cohen, A. Menzel, G. deBotton, Towards a physics-based multiscale modelling of the electromechanical coupling in electro-active polymers, Proceedings of the Royal Society/A, 472:2186, 2016.

[16] N. Cohen, A generalized electro-elastic theory of polymer networks, Journal of Mechanics and Physics of Solids, 110:173-191, 2018.

[17] M. Duduta, E. Hajiesmaili, H. Zhao, R. J. Wood, D. R. Clarke, Realizing the potential of dielectric elastomer artificial muscles, Proceedings of the National Academy of Sciences, 116(7):201815053, 2019. 
[18] Z. M. Dang, J. K. Yuan, J. W. Zha, T. Zhou, S. T. Li, G. H. Hu, Fundamentals, processes and applications of high-permittivity polymer-matrix composites, Progress in Materials Science, 57(4):660-723, 2012.

[19] A. Dorfmann. R. W. Ogden, Nonlinear electroelasticity, Acta Mechanica, 174(3-4):167-183, 2005.

[20] A. Dorfmann, R. W. Ogden, Nonlinear electroelastic deformations, Journal of Elasticity, 82(2):99127, 2006.

[21] A. Dorfmann. R. W. Ogden, Nonlinear electroelasticity: material properties, continuum theory and applications, Proceedings of the Royal Society/A, 473(2204):20170311, 2017.

[22] A. Dorfmann. R. W. Ogden, Nonlinear Theory of Electroelastic and Magnetoelastic Interactions, Springer-Verlag, Berlin, 2014.

[23] I. Diaconu, D. Dorohoi, C. Ciobanu, Electromechanical response of polyurethane films with different thickness, Romanian Journal of Physics, 53(1-2):91-97, 2006.

[24] I. Diaconu, D. Dorohoi, F. Topoliceanu, Electrostriction of a polyurethane elastomer-based polyester, IEEE Sensors Journal, 6(4):876-880, 2006.

[25] E.A. de Souza Neto, D. Peric, M. Dutko, D.R.J. Owen, Design of simple low order finite elements for large strain analysis of nearly incompressible solids, International Journal of Solids and Structures, 33(20-22): 3277-3296, 1996.

[26] C. Ellingford, C. Bowen, T. McNally, C. Wan, Intrinsically tuning the electromechanical properties of elastomeric dielectrics: A chemistry perspective, Macromol Rapid Communications, 39(18):1800340, 2018.

[27] P. Erbts, S. Hartmann, A. Düster, A partitioned solution approach for electro-thermo-mechanical problems, Archive of Applied Mechanics, 85:1075-1101, 2015.

[28] A. C. Eringen, G. A. Maugin, Electrodynamics of Continua, Springer-Verlag, 1990.

[29] D. J. Griffiths, Introduction to Electrodynamics, 3rd ed. Prentice Hall, 1998.

[30] Z. Gao, A. Tuncer, A. M. Cuitiño, Modeling and simulation of the coupled mechanical-electrical response of soft solids, International Journal of Plasticity, 27(10):1459-1470, 2011.

[31] A. Gil, R. Ortigosa, A new framework for large strain electromechanics based on convex multivariable strain energies: variational formulation and material characterisation, Computer Methods in Applied Mechanics and Engineering, 302:293-328, 2016.

[32] D. L. Hennan, S. A. Chester, K. Bertoldi, Modeling of dielectric elastomers: Design of actuators and energy harvesting devices, Journal of the Mechanics and Physics of Solids, 61(10):2047-2066, 2013.

[33] M. Hossain, P. Steinmann, Modelling electro-active polymers with a dispersion-type anisotropy, Smart Materials and Structures, 27(2):025010, 2018.

[34] M. Hossain, Modelling the curing process with a dispersion-type anisotropy in particle-filled electro-active polymers, Continuum Mechanics and Thermodynamics, DOI:10.1007/s00161-01900747-5, 2019.

[35] M. Hossain, D. K. Vu, P. Steinmann, Experimental study and numerical modelling of VHB 4910 polymer, Computational Materials Science, 59:65-74, 2012.

[36] M. Hossain, D. K. Vu, P. Steinmann, A comprehensive characterization of the electro-mechanically coupled properties of VHB 4910 polymer, Archive of Applied Mechanics, 84:523-537, 2015. 
[37] M. Hossain, P. Steinmann, More hyperelastic models for rubber-like materials: Consistent tangent operator and comparative study, Journal of the Mechanical Behaviour of Materials, 22(1-2):27-50, 2013.

[38] M. Hossain, N. Kabir, A. F. M. S. Amin, Eight-chain and full-network models and their modified versions for rubber hyperelasticity : A comparative study, Journal of the Mechanical Behaviour of Materials, 24(1-2):11-24, 2015.

[39] M. Itskov, V. N. Khiem, S. Waluyo, Electroelasticity of dielectric elastomers based on molecular chain statistics, Mathematics and Mechanics of Solids, 24(3):862-873, 2019.

[40] M. Jabareen, On the modeling of electromechanical coupling in electro-active polymers using the mixed finite element formulation, Procedia IUTAM, 12:105-115, 2015.

[41] A. Kovetz, Electromagnetic Theory, University Press, Oxford, 2000.

[42] S. J. A. Koh, C. Keplinger, T. Li, S. Bauer, Z. Suo, Dielectric elastomer generators: How much energy can be converted?, IEEE/ASME Transactions on mechatronics, 16(1):33-41, 2011.

[43] M. R. Kashani, S. Javadi, N. Gharavi, Dielectric properties of silicone rubber-titanium dioxide composites prepared by dielectrophoretic assembly of filler particles, Smart Materials and Structures, 19:1-7, 2010.

[44] A. Kumar, K. Patra, M. Hossain, On enhancement of electromechanical properties of silicone composites by adding high dielectric constant fillers and curing under high electric field, In Review, 2019.

[45] M. A. Keip, P. Steinmann, J. Schroeder, Two-scale computational homogenization of electroelasticity at finite strains, Computer Methods in Applied Mechanics and Engineering, 278:62-79, 2014.

[46] S. Klinkel, S. Zwecker, R. Mueller, A solid shell finite element formulation for dielectric elastomers, Journal of Applied Mechanics, 80(2):021026, 2013.

[47] L. Liu, An energy formulation of continuum magneto-electro-elasticity with applications, Journal of the Mechanics and Physics of Solids, 63:451-480, 2014.

[48] T. Li, G. Li, Y. Liang, T. Cheng, J. Dai, X. Yang, B. Liu, Z. Zeng, Z. Huang, Y. Luo, T. Xie, W. Yang, Fast-moving soft electronic fish, Science Advances, 3(4):e1602045, 2017.

[49] Z. S. Liao, M. Hossain, X. H. Yao, M. Mehnert, P. Steinmann, On thermo-viscoelastic experimental characterisation and numerical modelling of VHB polymer, International Journal of Non-Linear Mechanics, 118:103263, 2020.

[50] Z. S. Liao, X. H. Yao, L. H. Zhang, M. Hossain, J. Wang, S. Zang, Temperature and strain rate dependent large tensile deformation and tensile failure behavior of transparent polyurethane at intermediate strain rates, International Journal of Impact Engineering, 129:152-167, 2019.

[51] F. B. Madsen, A. E. Daugaard, S. Hvilsted, A. L. Skov, The current state of silicone-based dielectric elastomer transducers, Macromolecular Rapid Communications, 37(5):378-413, 2016.

[52] M. Mehnert, M. Hossain, P. Steinmann, On nonlinear thermo-electro-elasticity, Proceedings of the Royal Society/ A, 472(2190):20160170, 2016.

[53] M. Mehnert, M. Hossain and P. Steinmann, Numerical modeling of thermo-electro-viscoelasticity with field-dependent material parameters, International Journal of Non-Linear Mechanics, 126:1325, 2018. 
[54] M. Mehnert, M. Hossain, P. Steinmann, Numerical modelling of thermo-electro-viscoelasticity with field-dependent material parameters, International Journal of Non-Linear Mechanics, 106:13-24, 2018.

[55] M. Mehnert, M. Hossain, P. Steinmann, Towards a thermo-magneto-mechanical coupling framework for magneto-rheological elastomers, International Journal of Solids and Structures, 128:117-132, 2017.

[56] M. Mehnert, M. Hossain, P. Steinmann, Experimental and numerical investigations of the electroviscoelastic behavior of VHB 4905, European Journal of Mechanics -A/Solids, 77:103797, 2019.

[57] G. A. Maugin, Continuum Mechanics of Electromagnetic Solids, North Holland, 1988.

[58] S. Michel, X. Q. Zhang, M. Wissler, C. Löwe, G. Kovocs, A comparison between silicone and acrylic elastomers as dielectric materials in electroactive polymer actuators, Polymer International, 59:391-399, 2010.

[59] G. A. Maugin, A. C. Eringen, On the equations of the electrodynamics of deformable bodies of finite extent, Journal de Mécanique, 16:101-147, 1977.

[60] B. Nedjar, A finite strain modeling for electro-viscoelastic materials, International Journal of Solids and Structures, 97:312-321, 2016.

[61] B. Nedjar, A coupled BEM-FEM method for finite strain magneto-elastic boundary-value problems, Computational Mechanics, 59(5):795-807, 2017.

[62] R. Ortigosa, A. Gil, A new framework for large strain electromechanics based on convex multivariable strain energies: Finite Element discretisation and computational implementation, Computer Methods in Applied Mechanics and Engineering, 302:329-360, 2016.

[63] H. S. Park, Z. Suo, J. Zhou, P. A. Klein, A dynamic finite element method for inhomogeneous deformation and electromechanical instability of dielectric elastomer transducers, International Journal of Solids and Structures, 49(15-16): 2187-2194, 2012.

[64] H.S. Park, T. D. Nguyen, Viscoelastic effects on electromechanical instabilities in dielectric elastomers, Soft Matter, 9:1031-1042, 2013.

[65] H. S. Park, Q. Wang, X. Zhao, P. A. Klein, Electromechanical instability on dielectric polymer surface: modeling and experiment, Computer Methods in Applied Mechanics and Engineering, 260:4049, 2013.

[66] Y. H. Pao, Electromagnetic forces in deformable continua, Mechanics Today, (Nemat- Nasser, S., ed.), 4:209-306, Pergamon Press, Oxford, 1978.

[67] J. P. Pelteret, D. Davydov, A McBride, D. K. Vu, P. Steinmann, Computational electro- and magnetoelasticity for quasi-incompressible media immersed in free space, International Journal for Numerical Methods in Engineering, 108(11):1307-1342, 2016.

[68] R. Pelrine, R. Kornbluh, Q. Pei, J. Joseph, High-speed electrically actuated elastomers with strain greater than 100\%, Science, 287(5454):836-839, 2000.

[69] J. Qiang, H. Chen and B. Li, Experimental study on the dielectric properties of polyacrylate dielectric elastomer, Smart Material and Structures, 21:1-9, 2012.

[70] M. Rambausek, M.-A. Keip, Analytical estimation of non-local deformation-mediated magnetoelectric coupling in soft composites, Proceedings of the Royal Society/A, 474(2216), 2018.

[71] Z. Ullah, L. Kaczmarczyk, C. J. Pearce, Three-dimensional nonlinear micro/meso-mechanical response of the fibre-reinforced polymer composites, Composite Structures, 161:204-214, 2017. 
[72] Z. Ullah, L. Kaczmarczyk, S. A. Grammatikos, M. C. Everndenb, C. J. Pearce, Multi-scale computational homogenisation to predict the long-term durability of composite structures, Computers and Structures, 181:21-31, 2017.

[73] A. K. Sharma, M. Joglekar, A numerical framework for modeling anisotropic dielectric elastomers, Computer Methods in Applied Mechanics and Engineering, 344:402-420, 2019.

[74] A. K. Sharma, M. Joglekar, A computationally efficient locking free numerical framework for modeling visco-hyperelastic dielectric elastomers, Computer Methods in Applied Mechanics and Engineering, 352:625-653, 2019.

[75] P. Steinmann, M. Hossain, G. Possart, Hyperelastic models for rubber-like materials: Consistent tangent operators and suitability of Treloar's data, Archive of Applied Mechanics, 82(9):1183-1217, 2012.

[76] P. Saxena, M. Hossain, P. Steinmann, Nonlinear magneto-viscoelasticity of transversally isotropic magneto-active polymers, Proceedings of the Royal Society/A, 470:20140082, 2014.

[77] P. Saxena, D. K. Vu, P. Steinmann, On rate-dependent dissipation effects in electro-elasticity, International Journal of Non-Linear Mechanics, 62:1-11, 2014.

[78] S. Santapuri, R. L. Lowe, S. E. Bechtel, M. J. Dapino, Thermodynamic modeling of fully coupled finite-deformation thermo-electro-magneto-mechanical behavior for multifunctional applications, International Journal of Engineering Science, 72:117-139, 2013.

[79] S. Santapuri, Unified continuum modeling of fully coupled thermo-electro-magneto-mechanical behavior with applications to multifunctional materials and structures, $\mathrm{PhD}$ Thesis, Ohio State University, USA, 2012.

[80] S. Santapuri, S. E. Bechtel, Nondimensional characterization and asymptotic model development for multifunctional structures with application to load-bearing antennas, Smart Materials and Structures, 23(5):055013, 2014.

[81] M. H. B. M. Shariff, R. Bustamante, M. Hossain, P. Steinmann, A novel spectral formulation for transversely isotropic magneto-elasticity, Mathematics and Mechanics of Solids, 22(5):1158-1176, 2017.

[82] D. Sfyris, G. I. Sfyris, R. Bustamante, Nonlinear electro-magneto-mechanical constitutive modelling of monolayer graphene, Proceedings of the Royal Society/A, 472(2188), 2016.

[83] S. Skatulla, C. Sansour, A. Arockiarajan, A multiplicative approach for nonlinear electro-elasticity, Computer Methods in Applied Mechanics and Engineering, 245:243-255, 2012.

[84] S. Seifi, K. C. Park, H. S. Park, A staggered explicit-implicit finite element formulation for electroactive polymers, Computer Methods in Applied Mechanics and Engineering, 337:150-164, 2018.

[85] S. Seifi, H. S. Park, Computational modeling of electro-elasto-capillary phenomena in dielectric elastomers, International Journal of Solids and Structures, 87:236-244, 2016.

[86] L. R. G. Treloar, The Physics of Rubber Elasticity, Oxford University Press, USA, 1975.

[87] S. Thylander, Microsphere-based modeling of electro-active polymers, PhD Dissertation, Lund University, Sweden, 2016.

[88] S. Thylander, A. Menzel, M. Ristinmaa, An electro-mechanically coupled micro-sphere framework: application to the finite element analysis of electrostrictive polymers, Smart Materials and Structures, 21(9):094008, 2012.

[89] T. Schlögl, S. Leyendecker, Electrostatic-viscoelastic finite element model of dielectric actuators, Computer Methods in Applied Mechanics and Engineering, 299:421-439, 2016. 
[90] F. Vogel, On the modeling and computation of electro- and magneto-active polymers, PhD Dissertation, Friedrich-Alexander-University Erlangen-Nuremberg, Germany, 2014.

[91] F. Vogel, S. Goektepe, E. Kuhl, P. Steinmann, Modeling and simulation of viscous electro-active polymers, European Journal of Mechanics A/Solids, 48:112-128, 2014.

[92] R. Vertechy, G. Berselli, V. P. Castelli, M. Bergamasco, Continuum thermo-electro-mechanical model for electrostrictive elastomers, Journal of Intelligent Material Systems and Structures, 24:761-778, 2012.

[93] R. Vertechy, G. Berselli, V. P. Castelli, G. Vassura, Optimal design of Lozenge-shaped dielectric elastomer linear actuators: Mathematical procedure and experimental validation, Journal of Intelligent Material Systems and Structures, 21:503-515, 2010.

[94] D. K. Vu, G. Possart, P. Steinmann, Numerical modeling of non-linear electroelasticity, International Journal for Numerical Methods in Engineering, 70:685-704, 2007.

[95] D. K. Vu, P. Steinmann, A 2-D coupled BEM-FEM simulation of electro-elastostatics at large strain, Computer Methods in Applied Mechanics and Engineering, 199:1124-1133, 2010.

[96] D. K. Vu, P. Steinmann, On 3-D coupled BEM-FEM simulation of nonlinear electro-elastostatics, Computer Methods in Applied Mechanics and Engineering, 201:82-90, 2012.

[97] D. Zäh, C. Miehe Multiplicative electro-elasticity of electroactive polymers accounting for micromechanically-based network models, Computer Methods in Applied Mechanics and Engineering, 286:394-421, 2015.

[98] X. Zhao, Z. Suo, Electrostriction in elastic dielectrics undergoing large deformation, Journal of Applied Physics, 104(12):123530, 2008.

[99] S. Wang, M. Decker, D. L. Henann, S. A. Chester, Modeling of dielectric viscoelastomers with application to electromechanical instabilities, Journal of the Mechanics and Physics of Solids, 95:213-229, 2016.

[100] M. Wissler, E. Mazza, Mechanical behaviour of an acrylic elastomer used in dielectric elastomer actuators, Sensors and Actuators A, 134:494-504, 2007.

[101] Y. Zhao, J. W. Zha, L. J. Yin, S. T. Li, Y. Q. Wen, Z. M. Dang, Constructing advanced dielectric elastomer based on copolymer of acrylate and polyurethane with large actuation strain at low electric field, Polymer, 149:39-44, 2018.

[102] Y. Zhao, J. W. Zha, L. J. Yin, S. T. Li, Y. Q. Wen, Z. M. Dang, Remarkable electrically actuation performance in advanced acrylic-based dielectric elastomers without pre-strain at very low driving electric field, Polymer, 137:269-275, 2018.

[103] O. C. Zienkiewicz, R. L. Taylor, The Finite Element Method for Solid and Structural Mechanics, Elsevier Butterworth and Heinemann, Oxford, England, Sixth edition, 2005.

[104] K. J. Bathe, Finite Element Procedures, Prentice Hall Inc., New Jersey, 1996.

[105] E. A. de Souza Neto, D. Perić, D. R. J. Owen, Computational Methods for Plasticity, Theory and Applications, John Wiley and Sons, United Kingdom, 2008.

[106] J. Bonet, R. D. Wood, Nonlinear continuum mechanics for finite element analysis, Cambridge University Press, 1997.

[107] C. Kadapa, Mixed galerkin and least-squares formulations for isogeometric analysis, $\mathrm{PhD}$ thesis, College of Engineering, Swansea University, 2014. 
[108] C. Kadapa, W. G. Dettmer, D. Perić, Subdivision based mixed methods for isogeometric analysis of linear and nonlinear nearly incompressible materials, Computer Methods in Applied Mechanics and Engineering, 305:241-270, 2016.

[109] C. Kadapa, W. G. Dettmer, D. Perić, On the advantages of using the first-order generalised-alpha scheme for structural dynamic problems, Computers and Structures, 193:226-238, 2017.

[110] F. Brezzi and M. Fortin, Mixed and Hybrid Finite Element Methods, Springer-Verlag, 1991.

[111] C. Kadapa, Novel quadratic Bézier triangular and tetrahedral elements using existing mesh generators: Applications to linear nearly incompressible elastostatics and implicit and explicit elastodynamics, International Journal for Numerical Methods in Engineering, 117:543-573, 2019.

[112] C. Kadapa, Novel quadratic Bézier triangular and tetrahedral elements using existing mesh generators: Extension to nearly incompressible implicit and explicit elastodynamics in finite strains, International Journal for Numerical Methods in Engineering, 119:75-104, 2019.

[113] D. Chapelle and K. J. Bathe, The inf-sup test, Computers and Structures, 47:537-545, 1993.

[114] J. Liu, C. C. Foo, and Z. Zhang, A $3 D$ multi-field element for simulating the electromechanical coupling behavior of dielectric elastomers, Acta Mechanica Solida Sinica, 30:374-389, 2017.

[115] J. C. Simo, R. L. Taylor, K. S. Pister, Variational and projection methods for the volume constraint in finite deformation elasto-plasticity, Computer Methods in Applied Mechanics and Engineering, 51:177-208, 1985.

[116] F. Brezzi, K. J. Bathe, A discourse on the stability conditions for mixed finite element formulations, Computer Methods in Applied Mechanics and Engineering, 82:27-57, 1990.

[117] T. J. R. Hughes, The Finite Element Method: Linear Static and Dynamic Finite Element Analysis, Dover Publications, 2000.

[118] G. Scovazzi, B. Carnes, X. Zeng, S. Rossi, A simple, stable, and accurate linear tetrahedral finite element for transient, nearly, and fully incompressible solid dynamics: a dynamic variational multiscale approach, International Journal for Numerical Methods in Engineering, 106:799-839, 2016.

[119] G. Scovazzi, T. Song, X. Zeng, A velocity/stress mixed stabilized nodal finite element for elastodynamics: Analysis and computations with strongly and weakly enforced boundary conditions, Computer Methods in Applied Mechanics and Engineering, 325:532-576, 2017.

[120] N. Abboud, G. Scovazzi, Elastoplasticity with linear tetrahedral elements: A variational multiscale method, International Journal for Numerical Methods in Engineering, 115:913-955, 2018.

[121] M. Klassen, Numerical modeling for the static and dynamic analysis of nearly incompressible dielectric elastomers, $\mathrm{PhD}$ Thesis, University of Kaiserslautern, 2016.

[122] S. Li, W. Kim, T. Cheng, I. Oh, A helical ionic polymer-metal composite actuator for radius control of biomedical active stents, Smart Materials and Structures, 20:035008, 2011.

[123] A. Bijalwan, B. P. Patel, M. Marieswaran D. Kalyanasundaram, Volumetric locking free 3D finite element for modelling of anisotropic visco-hyperelastic behaviour of anterior cruciate ligament, Journal of Biomechanics, 73:1-8, 2018.

[124] D. S. Malkus, T. J. R. Hughes, Mixed finite element methods - Reduced and selective integration techniques: A unification of concepts, Computer Methods in Applied Mechanics and Engineering, 15:63-81, 1978.

[125] N. M. Newmark, A method of computation for structural dynamics, Journal of the Engineering Mechanics Division (ASCE), 85:67-94, 1959. 
[126] G. Guennebaud and B. Jacob and others, Eigen v3. http: // eigen. tuxfamily. org, 2010.

[127] HyperMesh. https://altairhyperworks. com/product/hypermesh

[128] PARDISO solver project. https: //www. pardiso-project. org/

[129] D. Kourounis and A. Fuchs and O. Schenk, Towards the Next Generation of Multiperiod Optimal Power Flow Solvers, IEEE Transactions on Power Systems, 99:1-10, 2018.

[130] W.R. Hamilton, On a General Method in Dynamics; by which the Study of the Motions of all free Systems of attracting or repelling Points is reduced to the Search and Differentiation of one central Relation, or characteristic Function, Philosophical Transactions of the Royal Society, Part II:247-308, 1834.

[131] W.R. Hamilton, Second Essay on a General Method in Dynamics, Philosophical Transactions of the Royal Society, Part I:95-144, 1835.

[132] V. I. Arnold, Mathematical Methods of Classical Mechanics, Second Edition, Springer-Verlag, New York, 1989.

[133] C. Lanczos, The Variational Principles of Mechanics, Fourth Edition, Dover Publications, Inc., New York, 1970.

[134] G. Holzapfel, Nonlinear solid mechanics: a continuum approach for engineering science, Wiley and Sons, 2001.

[135] L. A. Mihai, A. Goriely, How to characterize a nonlinear elastic material? A review on nonlinear constitutive parameters in isotropic finite elasticity, Proceedings of the Royal Society A, 473: $20170607,2017$.

[136] C. Kadapa, Newmark-beta method - disadvantages, https: // ckadapa. wordpress. com/2017/ 06/23/newmark-beta-method-disadvantages/, Wordpress blogpost, Accessed on 7th September 2020 .

[137] C. Kadapa, M. Hossain, A linearised consistent mixed displacement-pressure formulation for hyperelasticity, Mechanics of Advanced Materials and Structures, DOI:10.1080/15376494.2020.1762952, 2020.

[138] C. Geuzaine, J.-F. Remacle, Gmsh: a three-dimensional finite element mesh generator with builtin pre- and post-processing facilities, International Journal for Numerical Methods in Engineering, 79:1309-1331, 2009. 